\title{
Der Einfluss von Rotation auf Konvektion und Kristallisation eines binären eutektischen Systems in Hinblick auf den Erdkern
}

\author{
Dissertation \\ zur Erlangung des Doktorgrades \\ der Mathematisch-Naturwissenschaftlichen Fakultäten \\ der Georg-August-Universität zu Göttingen
}

vorgelegt von
Sabine Claßen
aus Rheydt

Göttingen 1999 


\section{7}

Referent: Prof. Dr. U. Christensen

Korreferent: Prof. Dr. H. Eckelmann

Tag der mündlichen Prüfung: 2. November 1999 


\section{Inhaltsverzeichnis}

1 Einleitung 5

2 Erdkern und Grenzregion innerer/äußerer Kern 10

2.1 Chemische Konvektion . . . . . . . . . . . . . . . . . . . . . 10

2.2 Grenzregion innerer/äußerer Kern . . . . . . . . . . . . . . . . . . . 11

2.3 Relevante Faktoren für die Morphologie der ICB $\ldots \ldots \ldots \ldots \ldots$

2.4 Anisotropie des inneren Erdkerns . . . . . . . . . . . . . . . . . 14

3 Allgemeine Eigenschaften des untersuchten Systems 15

3.1 Eutektische binäre Systeme . . . . . . . . . . . . . . . . . . 15

3.2 Wässrige Ammoniumchlorid-Lösung … . . . . . . . . . . . . . . . 19

3.3 Dendritisches Wachstum und Konvektion . . . . . . . . . . . . . 20

3.3 .1 Dendritisches Wachstum . . . . . . . . . . . . . . 20

3.3 .2 Kaminentstehung . . . . . . . . . . . . . . . . . . . . . 23

3.3.3 Konvektionsmoden . . . . . . . . . . . . . . . . 25

$\begin{array}{ll}4 \text { Versuchsautbau } & 27\end{array}$

4.1 Rotationsapparatur . . . . . . . . . . . . . . . . . . . . . . 27

$4.2 \quad$ Visualisierung der Strömung $\ldots \ldots \ldots$. . . . . . . . . . . . . . . . 31

5 Versuchsablaut 34

$5.1 \quad$ Vorbereitungen zu einem Experiment . . . . . . . . . . . . . . . . . 34

5.2 Ablaut eines Experiments $\ldots \ldots \ldots \ldots \ldots$

$\begin{array}{lll}6 & \text { Messgrößßen } & 38\end{array}$

6.1 Höhe der porösen Dendritenschicht . . . . . . . . . . . . . . . . . 38

6.2 Plume-Anzahl und-Durchmesser . . . . . . . . . . . . . . . . . 39

6.3 Dendritengrößße und Morphologie $\ldots \ldots \ldots$

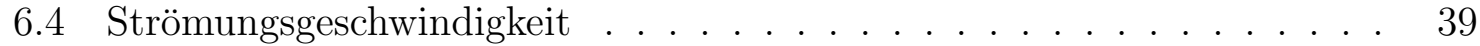

6.5 Konzentrationsmessung $\ldots \ldots \ldots \ldots \ldots$ 
7 Ergebnisse: Dendritisches Wachstum 43

7.1 Globale Beobachtungen des Wachstums . . . . . . . . . . . . . . . . . . 43

7.1.1 Starke Unterkühlung . . . . . . . . . . . . . . . . . . . 44

7.1 .2 Geringe Unterkühlung . . . . . . . . . . . . . . . . . 44

7.2 Protokaming . . . . . . . . . . . . . . . . . . . . . . 46

7.3 Eintluss der Rotation auf die Kristallschicht . . . . . . . . . . . . . . . 51

7.3 .1 Wachstumskinetit . . . . . . . . . . . . . . . 51

7.3 .2 Morphologie . . . . . . . . . . . . . . . 54

7.3.3 Abschätzung der Permeabilität und Porosität . . . . . . . . . . 55

7.3 .4 Kamine . . . . . . . . . . . . . . . . . . 58

$\begin{array}{lll}8 & \text { Ergebnisse: Konvektion } & 61\end{array}$

8.1 Protoplumes - Frühstadium der Konvektion . . . . . . . . . . . . . . . 61

8.2 Fingerkonvektion . . . . . . . . . . . . . . . . . . . . . . . . 63

8.3 plumes . . . . . . . . . . . . . . . . . . . . . . . 65

8.4 Einsatz der Plumekonvektion . . . . . . . . . . . . . . . . 66

8.5 Plumeanzahl beim Einsatz der Konvektion . . . . . . . . . . . . . . . . 69

8.6 Zeitliche Entwicklung der Plumekonvektion . . . . . . . . . . . . . . . . 69

8.7 Einfluss der Rotation auf die Strömung . . . . . . . . . . . . . . . . . . 72

8.7 .1 Zeitliche Entwicklung der Konvektion . . . . . . . . . . . . . . . 73

8.7 .2 Tröpfcheninstabilität . . . . . . . . . . . . . . . . . . 73

8.7 .3 Die Natur der Tröpfcheninstabilität . . . . . . . . . . . . . . . . 77

8.7 .4 Kleine Ekmanzahlen . . . . . . . . . . . . . . . . . 78

$\begin{array}{llr}9 & \text { Diskussion } & 81\end{array}$

$\begin{array}{ll}10 \text { Zusammenfassung } & 89\end{array}$

$\begin{array}{lr}\text { Literaturverzeichnis } & 91\end{array}$

\begin{tabular}{ll}
\hline Abbildungsverzeichnis & 100
\end{tabular}

$\begin{array}{ll}\text { Tabellenverzeichnis } & 101\end{array}$

\begin{tabular}{|ll|}
\hline A Liste der verwendeten Symbole und Abkürzungen & 102
\end{tabular}

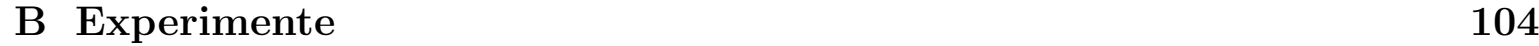

\begin{tabular}{ll}
\hline C Hardware & 106
\end{tabular} 


\section{Kapitel 1}

\section{Einleitung}

Unser Verständnis vom Aufbau der Erde ist trotz großer Fortschritte in der Geophysik noch lange nicht vollständig. Das Standardmodell für den Erdaufbau stützt sich im Wesentlichen auf Erkenntnisse, die uns die Seismologie liefert. Demnach ist die Erde in mehrere Kugelschalen (Abb. 1.1) gegliedert. Die Kruste bildet die äußerste, feste Schale, darunter liegt der Erdmantel aus Silikat-Gesteinen. Der innerste Bereich wird von einem größtenteils aus Eisen bestehenden Kern gebildet, dessen flüssige äußere Region durch eine Grenzzone (Inner Core Boundary, kurz: ICB) vom festen inneren Teil getrennt wird. Die ICB stellt auch eine Grenze für die Zusammensetzung dar, so enthält der äußere flüssige Teil einen größeren Anteil an leichten Elementen. Einige Auswirkungen der Vorgänge, die im Erdkern stattfinden, kann man auch noch auf der Erdoberfläche beobachten.

So ist heute allgemein anerkannt, dass das Erdmagnetfeld durch einen Dynamoprozess im Erdkern erzeugt wird. Angetrieben wird dieser Prozess durch Konvektion, also Strömungsvorgänge im elektrisch leitfähigen äußeren Kern. Bei diesen Bewegungen werden Ströme induziert, die zum Erdmagnetfeld führen. Man glaubt, dass die Konvektion den Großteil ihrer Antriebsenergie aus Prozessen erhält, die mit dem Ausfrieren (Kristallisation) des inneren Erdkerns zusammenhängen (Lister \& Buffett, 1995).

Eine entscheidene Rolle spielt hierbei die Grenze innerer/äußerer Kern, an der die Kristallisation stattfindet. Nach einem Modell von Loper \& Fearn (1983) wird dieser Bereich von einem porösen Netzwerk gebildet, dessen Matrix aus Eisenkristallen besteht. Die Zwischenräume sind mit konvektierendem, flüssigem Kernmaterial gefüllt, das um das auskristallisierte Eisen verarmt ist. Die daraus resultierenden Dichteunterschiede führen zu Auftrieb und lassen das Material in Richtung Kern/Mantel-Grenze 


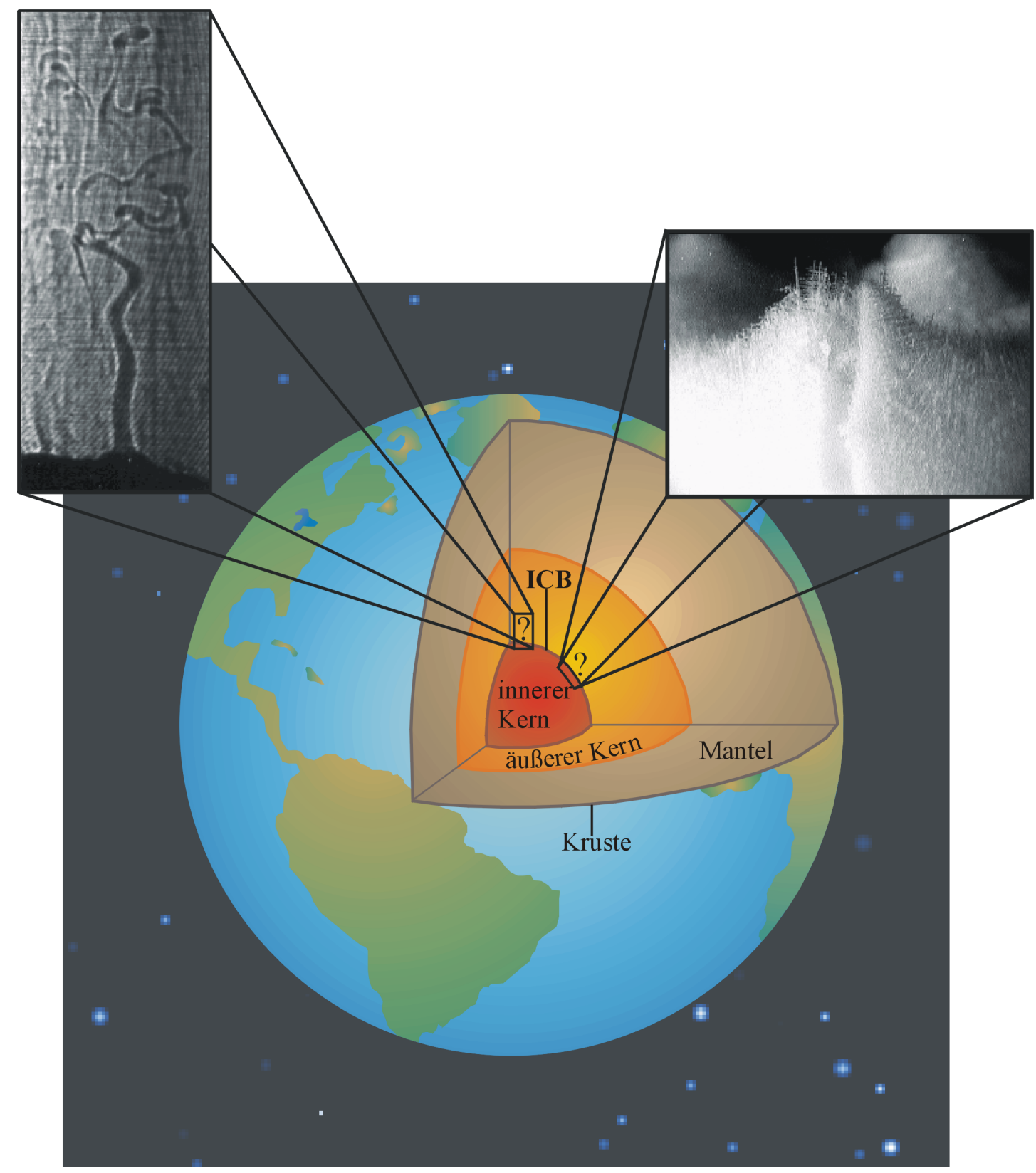

Abbildung 1.1: Innerer Aufbau der Erde

Zur Illustration der Vorstellung, die man von der Grenze innerer/äußerer Kern (ICB) hat, wurden Bilder der Kristallschicht und der Strömungsstrukturen aus dieser Arbeit eingefügt. 
aufsteigen. Dieser Effekt wird als chemische Konvektion bezeichnet. In Laborexperimenten mit Modellsystemen wie Ammoniumchlorid (z. B. Chen \& Chen, 1991) wird gezeigt, dass die Strömungen innerhalb der porösen Grenzschicht zur Ausbildung enger, kristallfreier Kanäle, sogenannte Kamine, führt (vgl. Abb. 1.1, rechtes Bild). Die Flüssigkeit wird in diesen Kanälen fokussiert, so dass sie als enger Flüssigkeitsschlauch (Plume) aus der Kristallschicht aufsteigt (Abb. 1.1, linkes Bild).

Aufgrund der hohen Rotationsrate der Erde können Corioliskräfte einen entscheidenden Einfluss auf die Dynamik haben, für die Atmosphäre und das Wettergeschehen ist dies manigfach belegt. Simulationen und Experimente zeigen die entscheidende Rolle der Corioliskraft für die Bewegungen im flüssigen äußeren Kern. Hier soll nun der Einfluss der Corioliskraft auf die Kristallisationsvorgänge am inneren Kern und die damit verbundenen Konvektionsströmungen untersucht werden. So ist denkbar, dass durch die Rotation zum Beispiel die Richtung des Kristallwachstums beeinflusst wird. Seismologische Untersuchungen geben Hinweise darauf, dass der innere Kern anisotrop ist (z. B. Creager, 1992). In diesem Zusammenhang beschreibt Anisotropie den Umstand, dass die elastischen Eigenschaften eines Körpers von seiner Orientierung abhängen. Im Erdkern äußert sich dieser Effekt in der schnelleren Ausbreitung der seismischen Wellen in Richtung der Rotationsachse durch den Kern als senkrecht dazu durch die Äquatorebene. Die Ausrichtung der Eisenkristalle zur Rotationsachse könnte eine Erklärung für diese Beobachtung sein.

Weiterhin besteht die Möglichkeit, dass sich die Strömungsstrukturen unter dem Einfluss der Rotation grundlegend ändern könnten und die Konvektion so ganz oder teilweise unterdrückt wird. So ist aus Rotationsexperimenten mit verschiedensten Randbedingungen (z. B. Zylindern oder Kugelschalen und mit verschiedenen Materialien) bekannt, dass sich die Strömungen grundlegend von der einfachen Rayleigh-Bénard Konvektion unterscheiden. So kann z. B. Busse (1970) zeigen, dass die Strömungsstrukturen in einer rotierenden Kugelschale von Säulen gebildet werden, die sich parallel zur Rotationsachse um eine innere beheizte Kugel anordnen. Dieses Strömungsmuster gehorcht in guter Näherung dem Taylor-Proudman Theorem (TPT), nachdem sich in einem stark rotierenden Kontainer das Geschwindigkeitfeld in Richtung der Rotationsachse nicht ändert. (Die zur Rotationsachse geneigten Ränder der Kugelschale verhindern die perfekte Erfüllung des Theorems, so das Sekundärströme auftreten.) Die dabei aufgeprägte Zylindersymmetrie unterdrückt z. B. den Einsatz kugelsymmetrischer thermischer Konvektion, so dass im Vergleich zum nichtrotierenden Fall stärker geheizt werden muss, um die Konvektion anzuregen (Chandrasekhar, 1961). 
Das Verständnis der chemischen Konvektion im Erdkern wird dadurch erschwert, dass der Ursprung der Konvektion möglicherweise in einer porösen Schicht liegt und nicht an einer planen Fläche entsteht. Diese poröse Schicht sollte die Konvektion beeinflussen, die wiederum durch den damit verbundenen Materialtransport auf die Kristallisation zurückwirken kann. Auch ist die Auswirkung von Corioliskräften auf das dendritische Wachstum und die chemische Konvektion noch wenig erforscht. Die Kristallisation und die Tendenz zur Bildung von Kaminen in der porösen Schicht scheint zwar nur wenig durch eine Rotationsbewegung um die vertikale Achse beeinflusst zu werden, wie Sample \& Hellawell (1984) und Neilson \& Incompera (1993b) in Laborexperimenten mit Ammoniumchlorid zeigen können. Die Konvektion wird jedoch in diesen Arbeiten nicht betrachtet. Cardin \& Olson (1992) wiederum untersuchen die rein chemische Konvektion in einer rotierenden Kugelschale, jedoch in einem System das keine kristalline Phase aufweist. Ihre Ergebnisse entsprechen im Prinzip den Taylorsäulen, die man für thermisch angetriebene Konvektion (siehe auch Carrigan \& Busse, 1983 und Busse, 1994) erhält. Die chemisch angetriebenen Strömungen, die zu den Säulen führen, sind jedoch wesentlich kleinskaliger als bei thermischer Konvektion unter vergleichbaren Parametern. Da Kristallisations- und Konvektionsverhalten bisher nur separat betrachtet wurden, steht eine einheitliche Untersuchung des gesamten wechselwirkenden Systems noch aus.

An diesem Punkt setzt die vorliegende Arbeit ein, die mit Blick auf die geophysikalischen Abläufe an der ICB die grundsätzlichen strömungs- und metallphysikalischen Vorgänge unter dem Einfluss von Rotation untersucht. Dazu werden sowohl das Kristallwachstum als auch die entstehenden Strömungsstrukturen unter dem Einfluss von Corioliskräften in Laborexperimenten betrachtet. Von besonderem Interesse sind hier vor allem der Einsatz der Plumekonvektion und die Strömungsstrukturen bei hohen Rotationsraten.

Als Modellsystem dient eine wässrige Ammoniumchlorid-Lösung $\left(\mathrm{NH}_{4} \mathrm{Cl}-\mathrm{H}_{2} \mathrm{O}\right)$, das ein geeignetes (pseudo-) binäres eutektisches System aus den beiden stabilen Phasen $\mathrm{NH}_{4} \mathrm{Cl}$ und $\mathrm{H}_{2} \mathrm{O}$ darstellt. Das System kristallisiert in einem von unten gekühlten rotierbaren Zylinder. Es ist jedoch nicht realisierbar die Laborexperimente unter Bedingungen durchzuführen, die mit dem Erdkern direkt vergleichbar sind. Darum wird versucht durch die Wahl eines großen Parameterbereichs die grundsätzlichen Abhängigkeiten der verschiedenen Vorgänge zu erfassen. Dazu werden die Anfangskonzentrationen der Lösung (24-28 gew\%- $\mathrm{NH}_{4} \mathrm{Cl}$ ), die Unterkühlung (5-25 K) sowie die Rotationsrate 
(Ekmanzahl]: 1,5 $\left.\cdot 10^{-4}-7,5 \cdot 10^{-6}\right)$ variiert.

Im nachfolgenden Kapitel wird der Stand der Forschung über den Erdkern und speziell der ICB ausführlicher behandelt. Daran anschließend werden die Eigenschaften des verwendeten Modellsystems beschrieben sowie das bereits bekannte Kristallisationsverhalten und die daraus resultierende chemische Konvektion erläutert. Die Ergebnisse zum dendritischen Wachstum und zur Strömungsdynamik sind Inhalt der Kapitel 7 und 8. In Kapitel 9 werden diese Ergebnisse diskutiert. Eine Diskussion der hier gewonnenen Ergebnisse sowie ihre Anwendung und ihre Bedeutung für das Verständnis der Vorgänge im Erdkern beschließt den Hauptteil dieser Arbeit.

\footnotetext{
${ }^{1}$ Ekmanzahl $=\frac{\text { viskose Kräfte }}{\text { Corioliskräfte }}$
} 


\section{Kapitel 2}

\section{Erdkern und Grenzregion innerer/äußerer Kern}

Der Erdkern hat einen Gesamtradius von 3485 km. Der äußere Erdkern hat eine Schichtdicke von $2270 \mathrm{~km}$ und besteht aus einer flüssigen Eisenlegierung mit etwa 10 \% leichteren Elementen, favorisierte Kandidaten sind Schwefel und Sauerstoff. Der innere Kern mit einem Radius von $1215 \mathrm{~km}$ besteht dagegen aus nahezu reinem, festem Eisen. Die Erde kühlt seit ihrer Entstehung ab, so dass der flüssige Kern langsam erkaltet und der feste Kern um wenige Zentimeter in 100 Jahren anwächst (Yoshida et al., 1996 und Labrosse et al., 1997). An der Grenzeregion zwischen dem inneren und dem äußeren Kern (ICB) kommt es durch die Auskristallisation von Eisen zu einer Anreicherung der leichten Elemente $(\mathrm{O}, \mathrm{S})$ in der Schmelze. Dies kann aufgrund der Dichteunterschiede zu aufsteigenden Strömungen - zur chemischen Konvektion - führen.

\subsection{Chemische Konvektion}

Nach Lister \& Buffet (1995) hat die chemische Konvektion einen Anteil von bis zu 80 \% an der gesamten Konvektion im Kern. Die verbleibenen 20 \% stammen aus thermischen Quellen, wie z. B. radioaktive Wärme oder latente Wärme. Buffett et al. (1996) geben für den angenommenen heutigen Wärmefluss aus dem Kern ein Verhältnis von zwei dritteln chemisch getriebener Konvektion zu einem drittel thermisch getriebener Konvektion an. In der jüngeren Erde, als der innere Kern kleiner und der Wärmefluss größer war, sollte die thermische Konvektion einen größeren Anteil gehabt haben. 


\subsection{Grenzregion innerer/äußerer Kern}

Die Grenzeregion zwischen innerem und äußerem Kern (ICB) wird vielfach kontrovers diskutiert. Die einzigen Messungen, die zur Klärung beitragen können, liefert die Seismologie. Die Interpretationen der seimischen Daten einzelner Autoren weichen jedoch mitunter stark voneinander ab. Die meisten Erdmodelle zeigen einen scharfen Anstieg der p-Wellengeschwindigkeit $v_{p}$ an der ICB von 10,22 $\frac{\mathrm{km}}{\mathrm{s}}$ auf 11,0 $\frac{\mathrm{km}}{\mathrm{s}}$ (Song \& Helmberger, 1992). Die Analyse der Amplituden und Modellierungen der Wellenform von PKP und PKiKP-Phasen lässt jedoch auf einen stärkeren Gradienten in der Scherwellengeschwindigkeit $v_{s}$ an der ICB schließen: $v_{s}>2,5 \frac{\mathrm{km}}{\mathrm{s}}$ (Shearer \& Masters, 1990), $2,5<v_{s}<3,0 \frac{\mathrm{km}}{\mathrm{s}}$ (Häge, 1983) und $v_{s}=3 \pm 1 \frac{\mathrm{km}}{\mathrm{s}}$ (Cummins \& Johnson, 1988a). Die mittlere s-Wellengeschwindigkeit im inneren Kern wird im Vergleich dazu auf 3,45 $\frac{\mathrm{km}}{\mathrm{s}}$ abgeschätzt (Shearer \& Masters, 1990). Diese Ergebnisse deuten darauf hin, dass es sich bei der ICB eher um eine Übergangszone als um eine scharfe Grenzfläche handelt, die maximale Mächtigkeit dürfte $5 \mathrm{~km}$ betragen (Cummins \& Johnson, 1988b).

Den wichtigsten Hinweis auf einen Übergangsbereich an der ICB liefert der Qualitätsfaktor $Q$, der das Verhältnis der Gesamtenergie einer Welle zum mittleren Energieverlust beim Fortschreiten um eine Wellenlänge in einem Medium beschreibt. $Q$ lässt damit Rückschlüsse auf die innere Reibung und somit auf die Eigenschaften des Materials zu. Der $Q$-Faktor für den äußeren Kern hat mit $Q>10^{4}$ (Cormier \& Richards, 1976) einen hohen Wert und damit ist die Dämpfung gering. Aus Analysen von PKP-Phasen erhält man $Q \sim 200$ (Doornbos, 1974) bis $Q \sim 360$ (Bhattacharyya et al., 1993) für den oberen Bereich des inneren Kerns und $Q \sim 1000$ in seinem Zentrum (Choy \& Cormier, 1983). Der geringe Wert von $Q$ an der Oberfläche des inneren Kerns lässt auf eine starke Dämpfung schließen, die eventuell von partiellen Schmelzen an der ICB hervorgerufen wird.

Ein Modell, das die Daten erklären könnte, stammt von Loper \& Roberts (1981), Loper \& Fearn (1983) und Loper (1983). Es berücksichtigt den dynamischen und thermodynamischen Zustand des Erdkerns. Demnach besteht die ICB aus einem porösen Netzwerk von kristallinen Eisen-Dendriten und konvektierender Flüssigkeit in den Zwischenräumen. Loper (1983) schätzt in seinem Modell die Dicke dieser Schicht mit etwa $300 \mathrm{~m}$. Eine solch geringe Mächtigkeit liegt unter dem seismischen Auflösungsvermögen, und ist somit in Übereinstimmung mit dem beobachteten scharfen Sprung der p-Wellengeschwindigkeit. 
Da demnach von der Seismologie keine detaillierten Informationen über den Aufbau der ICB zu erwarten sind, werden Laborexperimente durchgeführt, um die grundsätzlichen Vorgänge in einer vergleichbaren Grenzschicht zu untersuchen. Versuche mit metallischen Legierungen (Jackson et al., 1966; McDonald \& Hunt, 1969 und 1970; Sarazin \& Hellawell, 1988) und analogen Modellsystemen, wie z. B. eine wässrige Ammoniumchloridlösung (Tait \& Jaupart, 1989; Chen \& Chen, 1991) zeigen, dass die Aufströme der Konvektion in engen kristallfreien Kanälen oder Kaminen innerhalb der porösen Schicht fokussiert werden. Das führt zum Aufstieg von langgestreckten helikalen Plumes in die flüssige Phase oberhalb der Wachstumsfront.

Der Durchmesser eines Kamins und damit der Durchmesser eines Plumes ist nach unten durch den Abstand zweier benachbarter, primärer Dendritenarme begrenzt (z. B. Sarazin \& Hellawell, 1988 und Bergman et al., 1997). Aus Meteoritenanalysen von Esbensen \& Buchwald (1982) kann man den Abstand der Dendriten unter Erdkernbedingungen in einer Größenordnung von mehreren 10 Metern bis zu 100 m abschätzen. Bergman (1998) macht sogar Abschätzungen bis zu mehreren hundert Metern. Daraus folgt, dass eventuell in der Erde existierende Kamine einen Durchmesser von nicht weniger als 100 m besitzen sollten.

\subsection{Relevante Faktoren für die Morphologie der ICB}

Der Einfluss uniaxialer Verdichtung durch Sedimentation innerhalb einer porösen Schicht wird von Sumita et al. (1996) auf die Vorgänge im Erdkern angewendet. Verursacht durch den Dichtekontrast $\Delta \rho=600 \frac{\mathrm{kg}}{\mathrm{m}^{3}}$ zwischen der flüssigen und festen Phase kann es innerhalb der Schicht zum Absinken von schwererem abgebrochenen festem Material und dadurch zu einer Beeinflussung der Porosität kommen. Berücksichtigt man die herrschende Schwerebeschleunigung $g=4,4 \frac{m}{s^{2}}$, die mittlere Wachstumgeschwindigkeit $v_{g}$ des inneren Kerns von $v_{g} \sim 10^{-12} \frac{\mathrm{m}}{\mathrm{s}}$ und die dynamische Viskosität $\mu_{s}$ des festen, inneren Kerns $10^{16}<\mu_{s}<10^{20}$ Pas, so erhält man eine Abschätzung über die Mächtigkeit $H_{c}$ der porösen Grenzschicht $H_{c} \sim \sqrt{\mu_{s} v_{g} /(\Delta \rho g)}$ von $10 \mathrm{~m}<H_{c}<1000$ m für den Erdkern (Sumita et al., 1996).

Der Einfluss von Lorentzkräften ist ebenfalls ein wichtiger Faktor, der die Strömung in einer porösen Schicht an der Grenze zum inneren Erdkern beeinflussen kann. Berg- 
man \& Fearn (1994) diskutieren eine mögliche Unterdrückung der Kaminentstehung, falls die Lorentzkraft gegenüber den viskosen Kräften innerhalb der porösen Schicht dominiert. Dieser Fall tritt ein, wenn die Chandrasekarzahl $Q_{m}$ (Verhältnis der Lorentzkraft zu den viskosen Kräften) für diese Schicht $Q_{m}=\sigma B^{2} \Pi / \mu_{l} \gg 1$ wird $(\sigma$ : elektrische Leitfähigkeit, $B$ : magnetische Feldstärke, $\Pi$ : Permeabilität, $\mu_{l}$ : dynamische Viskosität des flüssigen Eisens). Die Bestimmung von $Q_{m}$ für den Kern ist jedoch trotz guter Abschätzungen für $\sigma$ und $\mu_{l}$ schwierig, da die Permeabilität auf Grund der Verdichtung von oben nach unten abnimmt und $B$ an der ICB unbekannt ist. Somit ist auch die Möglichkeit der aufsteigenden Flüssigkeit, innerhalb der Grenzschicht Kamine auszubilden, tiefenabhängig. Die Kombination der beiden Effekte - Sedimentation und Lorentzkraft - legt nahe, dass Kamine in konvektiven Gebieten mit einer hohen Permeabilität entstehen können.

Auf Grund der hohen Erdrotation ist die Corioliskraft eine weitere wichtige Kraft im Kern. Mit Hilfe der Rossbyzahl $\epsilon$ und der Ekmanzahl $E$ kann man den Einfluss von Corioliskräften auf die Dynamik im Erdkern abschätzen. Die Rossbyzahl gibt das Verhältnis von Advektion zu Corioliskräften wider, und die Ekmanzahl ist definiert als das Verhältnis von viskosen Kräften zur Corioliskraft:

$$
\begin{gathered}
\epsilon=\frac{U}{\Omega L} \\
E=\frac{\nu}{2 \Omega L^{2}}
\end{gathered}
$$

mit der Strömungsgeschwindigkeit $U$, der kinematischen Viskosität $\nu$, der Winkelgeschwindigkeit $\Omega$ und einer typischen Längenskala $L$. Sind $\epsilon \gg 1$ und $E \gg 1$, dann spielt die Rotation keine entscheidende Rolle für die Konvektionsdynamik. Sind $\epsilon \ll 1$ und $E \ll 1$, ist die Corioliskraft die dominierende Kraft und die Strömung wird stark beeinflusst. Melchior (1986) schätzt die Rossbyzahl und die Ekmanzahl für den Erdkern auf $\epsilon=4 \cdot 10^{-6}$ bis $4 \cdot 10^{-7}$ bzw. $E=2 \cdot 10^{-10}$ bis $10^{-15}$. Die Strömungsdynamik des Kerns wird somit wesentlich durch die Corioliskraft beeinflusst.

Das Verhältnis der Corioliskraft zur Lorentzkraft wird durch die Elsasserzahl $\Lambda=B^{2} / \rho \mu \sigma \Omega$ ausgedrückt. Im Erdkern sind diese Kräfte vergleichbar $(\Lambda \approx 1)$. 


\subsection{Anisotropie des inneren Erdkerns}

Seismologische Studien können anhand von Eigenschwingungen der Erde (z. B. Woodhouse et al., 1986 und Tromp, 1993) und Wellenphasen (z. B. Poupinet et al., 1983 und Morelli et al., 1986) zeigen, dass der innere Erdkern elastisch anisotrop ist. Die Anisotropie äußert sich durch eine um etwa $3 \%$ schnellere Ausbreitung der p-Welle entlang der Rotationsachse als in der Äquatorebene, wobei die Anisotropie mit der Tiefe zunimmt (Su \& Dziewonski, 1995 und Song, 1996).

Die bevorzugte Erklärung für die Anisotropie ist die Ausrichtung der Eisenkristalle entlang der Rotationsachse, da Eisen unter Kernbedingungen höchstwahrscheinlich hexagonal dichtest gepackt ist (Stixrude \& Cohen, 1995 und Saxena et al., 1995) und hexagonale Kristalle diese Art der Anisotropie aufweisen. Diskutiert werden im Prinzip zwei verschiedene Gründe für die Anisotropie:

1. Ausrichtung der Kristalle durch eine großskalige thermische Konvektion im inneren Kern (Jeanloz \& Wenk, 1988 und Romanowicz et al., 1996).

2. Die Kristalle werden bereits beim Wachstum durch Einwirken des Magnetfeldes (Karato, 1993) oder auf Grund von Corioliskräften, die die Richtung des Wärmeflusses beeinflussen (Stevenson, 1996; Bergman, 1997 und Bergman, 1999), ausgerichtet. Die genaue Herkunft der Anisotropie im inneren Erdkern ist jedoch nach wie vor unklar. 


\section{Kapitel 3}

\section{Allgemeine Eigenschaften des untersuchten Systems}

Im folgenden Kapitel werden zunächst die Vorgänge erläutert, die zu chemisch getriebener Konvektion während der Auskristallisation in einem eutektischen System führen können. Im Weiteren wird das hier verwendete Arbeitsmedium, eine wässrige Ammoniumchlorid-Lösung, vorgestellt. Der dritte Teil beinhaltet die wichtigsten Erkenntnisse, die bisher über das dendritische Wachstum und die daraus resultierende chemisch getriebene Konvektion bekannt sind. Diese Ergebnisse konnten im Verlauf der Arbeit nachvollzogen und bestätigt werden, sind jedoch nicht Teil der primären Fragestellung, weshalb sie an dieser Stelle zusammengefasst werden.

\subsection{Eutektische binäre Systeme}

In Abbildung 3.1 A ist ein Ausschnitt aus dem idealisierten Phasendiagramm eines eutektischen Systems der Komponenten A und B dargestellt. Unterschreitet die flüssige Legierung der anfänglichen Konzentration $C_{o}$ die Liquidustemperatur $T_{L}$, fängt die Ausscheidung der festen Phase mit einer Konzentration an, die dem Solidus bei dieser Temperatur entspricht. Bei weiterer Abkühlung im Gleichgewicht wandert die Konzentration der festen Phase entlang des Solidus auf die Konzentration $C_{S}$ zu und die der flüssigen entlang des Liquidus auf $C_{L}$. Hierbei beschreibt das Hebelgesetz die Abnahme des flüssigen Anteils und die Zunahme des festen Anteils, wie sie durch die Massenerhaltung festgelegt wird. Bei Veränderung der Anfangskonzentration $C_{o}$ verändert sich nur das Verhältnis fest/flüssig, jedoch nicht die Zusammensetzung dieser Phasen, die- 
se ist durch die Temperatur und den Solidus bzw. Liquidus festgelegt. (In den hier durchgeführten Experimenten wurde auch kein Einfluss der Anfangskonzentration beobachtet, der entscheidende Parameter ist hier die Unterkühlung.) Wird das System soweit heruntergekühlt, dass die eutektische Temperatur $T_{E}$ unterschritten wird, erhält man eine Entmischung der Restschmelze in die beiden festen Randphasen A und B. Aufgrund der langsamen Festkörperdiffusion stellt sich bei Abkühlung mit einer endlichen Geschwindigkeit kein Konzentrationsgleichgewicht in der festen Phase ein und die zuerst kristallisierte feste Phase enthält eine höhere Konzentration an A als im Gleichgewicht (Details siehe Haasen, 1994, S. 60).

In den Diagrammen B bis E ist die Situation bei einer gerichteten Erstarrung mit einem linearen Temperaturprofil (entsprechend der y-Achse in Diagramm A) über die Höhe dargestellt. Vor der festen Phase wird entsprechend dem Phasendiagramm ein Phasengemisch beobachtet (Abb. 3.1 B), das aus festen, kristallinen Dendriten besteht, zwischen denen sich noch flüssige Phase befindet. (Das dendritische Wachstum wird in Kapitel 3.3.1 erläutert). Für die Konvektion sind die Profile C-D relevant. Existieren in einem System Bereiche mit unterschiedlichen Temperaturen $T$ und/oder Variationen in der Konzentration $C$, so kann dies unter folgenden Bedingungen zu Konvektion führen: Die thermisch und chemisch induzierten Dichtegradienten werden im Wesentlichen durch den thermischen und chemischen Volumenausdehnungskoeffizienten bestimmt. Der thermische Ausdehnungskoeffizient $\alpha_{T}$ ist immer positiv, wohingegen der chemische Ausdehnungskoeffizient $\beta$ beide Vorzeichen haben kann.

Damit es zur Konvektion kommen kann, muss es eine instabile Schichtung von Material geringerer Dichte unterhalb einer dichteren Schicht geben. Von besonderem Interesse sind Geometrien, in denen die vertikalen Dichtegradienten $\left(\frac{\delta \rho}{\delta z}\right)$ bezogen auf $T$ und $C$ nicht nur verschieden voneinander sind, sondern auch umgekehrte Vorzeichen besitzen:

$$
-\left(\frac{\delta \rho}{\delta z}\right)_{T}<\left(\frac{\delta \rho}{\delta z}\right)_{C}
$$

Eine solche Situation findet man bei Kristallisationsvorgängen mit einem nach unten gerichteten Wärmefluss und Kristallwachstum antiparallel zum Schwerevektor. Der vertikale Temperaturgradient $\frac{\delta T}{\delta z}$ ist positiv (Abb. 3.1 D), zusammen mit dem thermischen Ausdehnungskoeffizienten führt dies zu einem negativen Dichtegradienten (Abb. 3.1 E): $\left(\frac{\delta \rho}{\delta z}\right)_{T}<0$. Die Flüssigkeit ist somit thermisch stabil.

Unterhalb der Front steigt die Konzentration der Flüssigkeit innerhalb des Dendritennetzwerkes gemäß des Phasendiagramms und umgekehrt proportional zur Steigung 

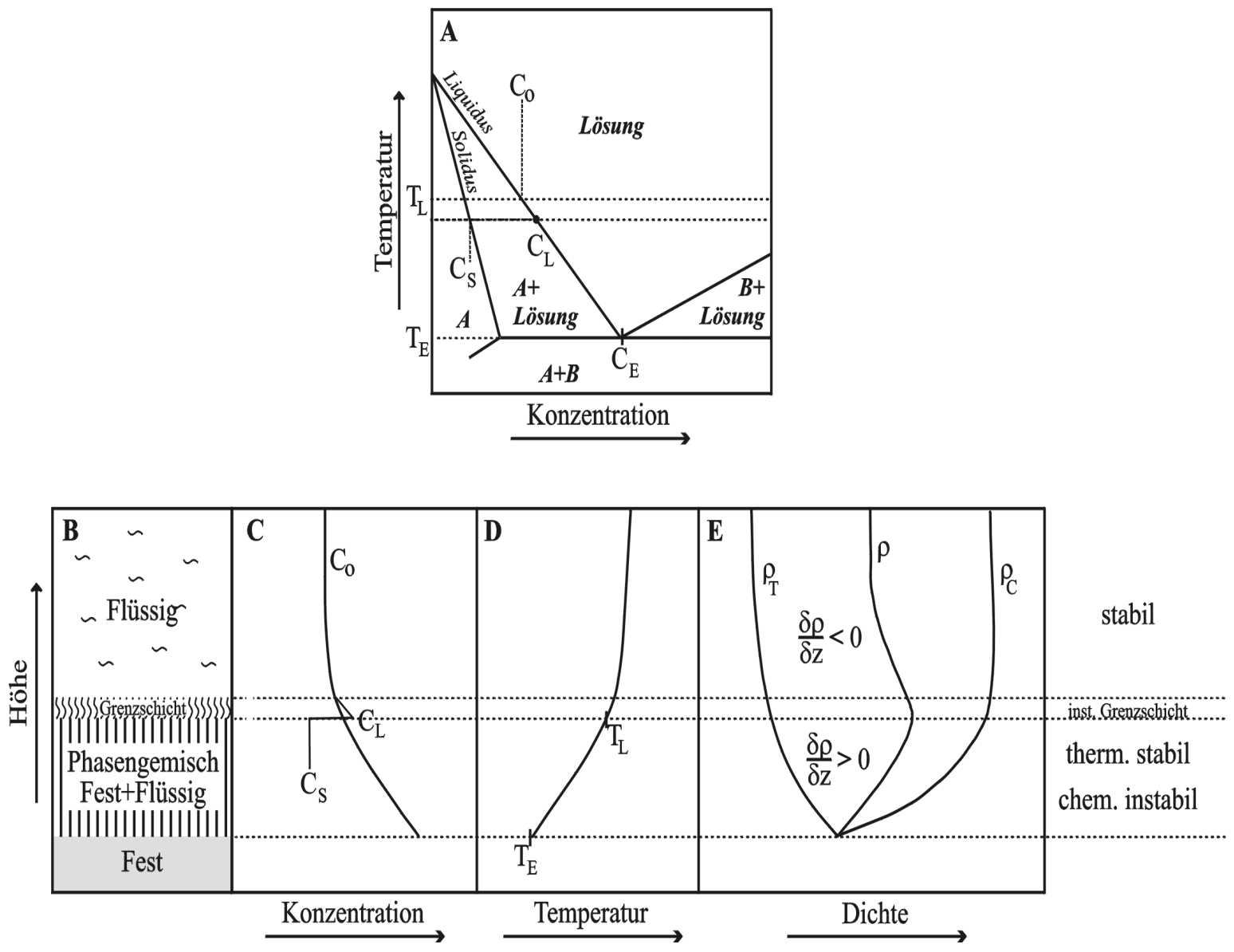

\section{Abbildung 3.1: Gerichtetes Wachstum eines eutektischen Systems}

(nach Hellawell et al., 1993 und Worster, 1997)

A: Ausschnitt eines idealisierten eutektischen Phasendiagramms, B: Schichtung der unterschiedlichen Phasen, C: vertikales Konzentrationsprofil, D: vertikales Temperaturprofil, E: vertikale Dichteprofile auf Grund reiner thermischer Einflüsse $\left(\rho_{T}\right)$, reiner Konzentrationseinflüsse $\left(\rho_{C}\right)$ und resultierendes gesamtes Dichteprofil $(\rho)$ 
des Liquidus $\Gamma=\frac{\delta C}{\delta T}$ an (Abb. $\left.3.1 \mathrm{C}\right)$ :

$$
\frac{\delta C}{\delta z}=\frac{\delta C}{\delta T} \times \frac{\delta T}{\delta z}
$$

Mit einem positiven chemischen Ausdehnungskoeffizienten kann der chemisch bedingte vertikale Dichtegradient positiv werden (Abb. 3.1 E).

$$
\left(\frac{\delta \rho}{\delta z}\right)_{C}=\frac{\delta \rho}{\delta C} \times \frac{\delta C}{\delta z}>0
$$

Für den gesamten vertikalen Dichtegradienten $\left(\frac{\delta \rho}{\delta z}\right)_{C, T}$ kann sich damit ein Vorzeichenwechsel ergeben und durch die instabile Schichtung kann es zur Konvektion kommen.

Ob es zum Einsatz der Konvektion kommt, hängt von den relativen Größenordnungen der Volumenausdehnungskoeffizienten, der Liquidussteigung und dem Temperaturgradienten ab, so dass der positive Dichtegradient unterhalb der Wachstumsfront den negativen Gradienten oberhalb übersteigt. Der chemisch erzeugte Auftrieb kann dann so groß werden, dass die viskosen Kräfte und thermische Diffusion des Systems überwunden werden.

Dieses Problem kann somit auf eine modifizierte Version der Rayleigh-Bénard Konvektion mit einem thermo-chemischen Ansatz zurückgeführt werden (z. B. Emms \& Fowler, 1994 und Huppert, 1990). Das System wird u. a. durch die dimensionslosen Zahlen: Prandtlzahl Pr und Lewiszahl Le beschrieben.

$$
\begin{array}{lll}
\operatorname{Pr}=\frac{\nu}{\kappa_{T}} & : & \frac{\text { viskose Kräfte }}{\text { therm. Diffusion }} \\
L e=\frac{\kappa_{T}}{\kappa_{C}} & : & \frac{\text { therm. Diffusion }}{\text { chem. Diffusion }}
\end{array}
$$

$\kappa_{C}$ : chem. Diffusionskoeffizient

Da chemische Konvektion in vielen verschiedenen Bereichen auftritt, wie Konvektion in Ozeanen (Salzlösungen), Magmenkammern (Laven) und im Erdkern (flüssige Metalle), ist in der Erde ein weiter Bereich an Prandtlzahlen realisiert, von etwa $2 \cdot 10^{-2}$ in Metallen bis etwa 10 in Salzlösungen und über 1000 in Laven. Die Lewiszahlen liegen enger zusammen mit $\approx 10^{3}$ für Metalle und $\approx 10^{2}$ für Salzlösungen. 


\subsection{Wässrige Ammoniumchlorid-Lösung}

Das verwendete Arbeitsmedium ist eine wässrige Ammoniumchlorid-Lösung $\left(\mathrm{NH}_{4} \mathrm{Cl}\right.$ $\mathrm{H}_{2} \mathrm{O}$ ). Jackson et al. (1966) zeigen, dass $\mathrm{NH}_{4} \mathrm{Cl}-\mathrm{H}_{2} \mathrm{O}$ analog $\mathrm{zu}$ metallischen Legierungen auskristallisiert. Auf Grund der Lichtdurchlässigkeit der Dendriten und der Transparenz der Flüssigkeit wird die Lösung vielfach zur direkten visuellen Untersuchung der Kristallisations- und Konvektionsvorgänge eutektischer Systeme eingesetzt.

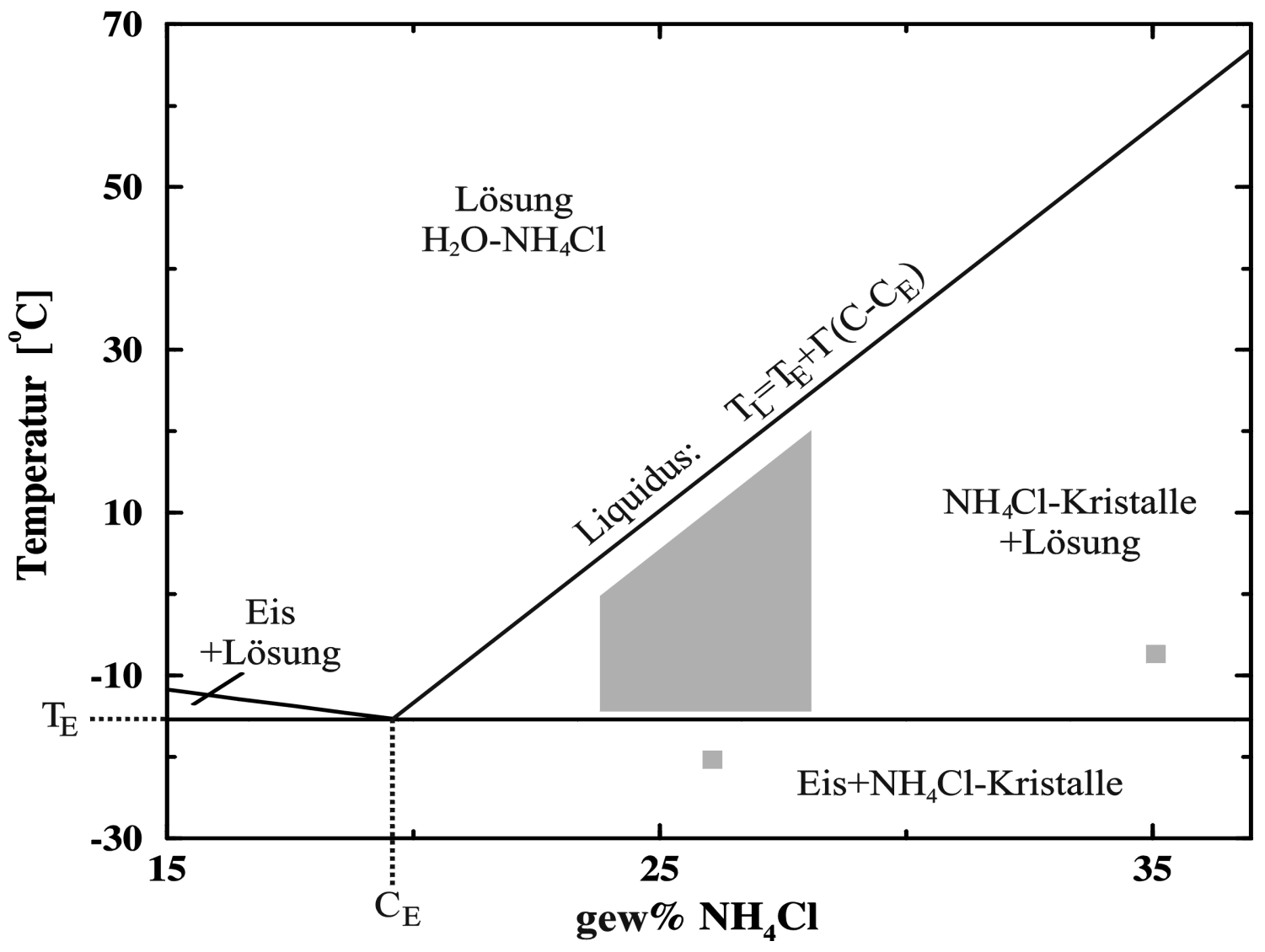

Abbildung 3.2: Phasendiagramm von $\mathbf{N H}_{4} \mathbf{C l}-\mathbf{H}_{2} \mathrm{O}$

Relevanter Ausschnitt aus dem Phasendiagramm mit dem Arbeitsbereich (grau) der Experimente. Die Kennzeichnung des Bereichs richtet sich nach der anfänglichen Konzentration $C_{o}$ der Lösung und der Kühltemperatur $T_{1}$ während des Versuchs.

Es handelt sich um ein einfaches, binäres eutektisches System mit vernachlässigbaren Randlöslichkeiten im Phasendiagramm (Abb. 3.2). Die Steigungen $\Gamma$ der Liquiduskurven können in guter Näherung als konstant angesehen werden. Die Steigung für den untersuchten Bereich (grau) und alle anderen Daten für das System können der Tabelle 3.1 entnommen werden. 


\begin{tabular}{|l|r|}
\hline Parameter & Wert \\
\hline \hline Steigung des Liquidus $\Gamma=\frac{d T}{d C}$ & $4,75 \frac{K}{\%}$ \\
\hline Eutektische Temperatur $T_{E}$ & $-15,4^{\circ} C$ \\
\hline Eutektische Konzentration $C_{E}$ & $19,7 \%$ \\
\hline Thermischer Diffusionskoeffizient $\kappa_{T}$ & $1,336 \cdot 10^{-3} \frac{\mathrm{cm}^{2}}{\mathrm{~s}}$ \\
\hline Chemischer Diffusionskoeffizient $\kappa_{C}$ & $1,7 \cdot 10^{-5} \frac{\mathrm{cm}^{2}}{\mathrm{~s}}$ \\
\hline Thermischer Expansionskoeffizient $\alpha_{T}$ & $3,832 \cdot 10^{-4} \mathrm{~K}^{-1}$ \\
\hline Chemischer Expansionskoeffizient $\beta$ & $2,82 \cdot 10^{-3} \%^{-1}$ \\
\hline Kinematische Viskosität $\nu$ & $1,2 \cdot 10^{-2} \frac{\mathrm{cm}^{2}}{\mathrm{~s}}$ \\
\hline Dichte der Lösung $\rho_{L}$ & $1,08 \cdot 10^{-3} \frac{\mathrm{kg}}{\mathrm{cm}^{3}}$ \\
\hline Dichte von NH${ }_{4}$ Cl $\rho_{S}$ & $1,53 \cdot 10^{-3} \frac{\mathrm{kg}}{\mathrm{cm}^{3}}$ \\
\hline Brechungsindex $n_{D}:$ & $1,7 \cdot 10^{-4} \mathrm{~K}^{-1}$ \\
Thermischer Koeffizient $n_{T}$ & $1,8 \cdot 10^{-3} \mathrm{gew}^{-1}$ \\
Chemischer Koeffizient $n_{C}$ & 79 \\
\hline Lewiszahl $L e$ & 9 \\
\hline Prandtlzahl $\operatorname{Pr}$ & \\
\hline
\end{tabular}

Tabelle 3.1: Physikalische Parameter einer 26 gew\% $\mathbf{N H}_{4} \mathbf{C l}-\mathbf{H}_{2} \mathrm{O}$ Lösung (nach Chen et al., 1994, Hellawell et al., 1993, Sarazin \& Hellawell, 1988)

Der Arbeitsbereich ist sowohl physikalischen als auch experimentellen Grenzen unterworfen. Ab einer Konzentration $C$ von 30 gew\%- $\mathrm{NH}_{4} \mathrm{Cl}$ mit der Liquidustemperatur $T_{L} \approx 33,5^{\circ} \mathrm{C}$ wird die experimentelle Handhabung bereits aufwendig und ab etwa 55 gew\%- $\mathrm{NH}_{4} \mathrm{Cl}$ beginnt die Lösung zu kochen. Der interessante Temperaturbereich ist nach oben zum einen durch den Liquidus beschränkt und zum anderen dadurch, dass sich Kamine erst ab einer Unterkühlung $\Delta T$ von etwa $5 \mathrm{~K}$ ausbilden. Da in dieser Arbeit das poröse Phasengemisch von zentralem Interesse ist, wird die eutektische Temperatur nur in einem Fall unterschritten.

\subsection{Dendritisches Wachstum und Konvektion}

\subsubsection{Dendritisches Wachstum}

Der Wachstumsvorgang bei der Erstarrung einer Schmelze hängt vom Abtransport der freigesetzten latenten Kristallisationswärme ab. Die latente Wärme wird an der Wachstumsfront fest/flüssig frei und muss von dort weggeführt werden. Die Art des Wärme- 
transportes bestimmt die Form der Kristalle. Wird die Wärme über den Festkörper abgeführt, können Ausbuchtungen nicht lokal in die Schmelze hineinwachsen, da sie durch die freigesetzte Kristallisationswärme wieder aufschmelzen würden. In Abbildung 3.3 A sind die so entstehende stabile planare Grenzfläche und das polygone Gefüge dargestellt. Beim Abtransport der Wärme über die Schmelze ist die Grenzfläche dagegen instabil, feste Ausbuchtungen wachsen in die Schmelze hinein und somit können Dendriten entstehen (Haasen, 1994, Seite 55ff). Neben diesem Effekt, der auch in reinen Metallen auftreten kann, kommt bei Legierungen noch die konstitutionelle (d. h. durch die Zusammensetzung verursachte) Unterkühlung als weitere Ursache für eine instabile Grenzfläche hinzu (Haasen, 1994, Seite 66ff). Da bei der Kristallisation zunächst Material mit der Konzentration $C_{S}$ kristallisiert, reichert sich in der Schmelze die überschüssige Komponente B an. Durch Diffusion von B entsteht ein Konzentrationsprofil vor der festen Oberfläche, aus dem man mit dem Liquidus des Phasendiagramms den gekrümmten Verlauf der Liquidustemperatur vor der Grenzfläche berechnen kann. Nimmt man einen linearen Temperaturanstieg vor der festen Oberfläche an, so schneidet i. A. die tatsächliche Temperatur die Liquidustemperatur. Bis zu diesem Ort liegt die Temperatur der Schmelze unter der Liquidustemperatur, die unterkühlte Schmelze ist instabil und kann kristallisieren.

Instabile Grenzflächen führen dazu, dass Ausstülpungen in die Schmelze hineinwachsen können. Die so entstehenden neuen Grenzflächen können dann wieder instabil werden, so dass sich wiederum neue Ausstülpungen senkrecht zur ursprünglichen Wachsstumsrichtung bilden usw. Die so entstehenden Kristallite mit zahlreichen tannenbaum-ähnlichen Verästelungen nennt man Dendrite (Abb. 3.4). Die detaillierten Wachstumsmechanismen mit der Singularität an der Spitze der Dendriten sind Gegenstand aktueller Forschung (als Einführung siehe Kurz \& Fisher, 1989, als Überblick siehe Müller-Krumbhaar \& Kurz, 1991), auf die hier nicht im Detail eingegangen werden soll.

Bei einer gerichteten Kristallisation, z. B. einer kontrollierten Abkühlung von unten, wachsen die primären Dendriten ohne andere äußere Kräfte nahezu parallel nebeneinander. Ihre sekundären, tertiären etc. Verästelungen wachsen aufgrund der anisotropen Kristallisationsgeschwindigkeiten häufig in kristallographisch niedrig indizierte Richtungen. So wachsen kubische Systeme (wie z. B. $\mathrm{NH}_{4} \mathrm{Cl}$ ) im Allgemeinen bevorzugt in $<100>$ und hexagonale in <0001> (Kleber (1956), S. $193 \mathrm{f}$ ), wie es auch für Fe unter Kernbedingungen der Fall sein könnte. Auf diese Weise entsteht das komplexe fest/flüssig dendritische Gefüge (Abb. 3.3 B). 
A
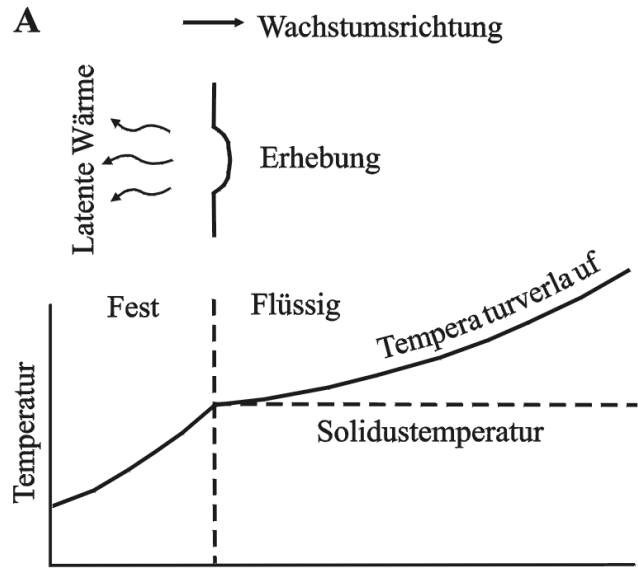

Abstand von der Grenzfläche fest/flüssig
B $\quad \longrightarrow$ Wachstumsrichtung

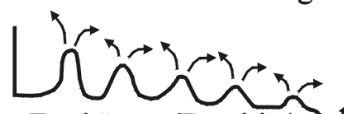

Festkörper (Dendrite) $>$ Schmelze
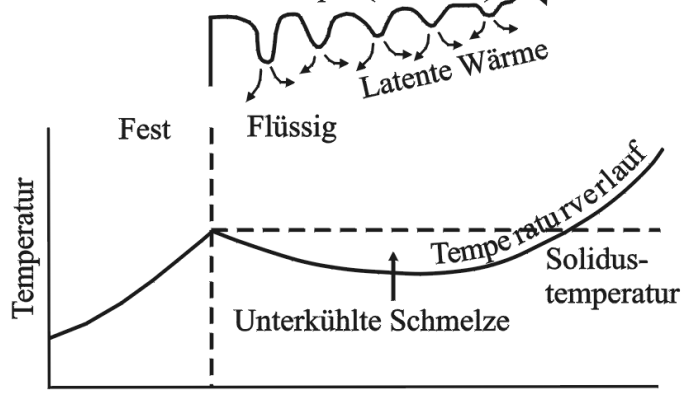

Abstand von der Grenzfläche fest/flüssig

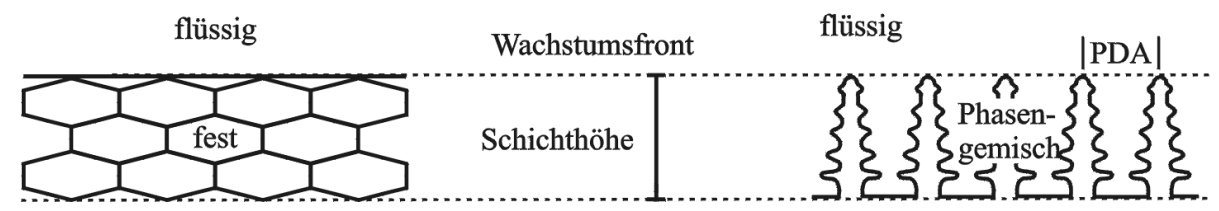

\section{Abbildung 3.3: Planares und Dendritisches Kristall-Wachstum}

für ein Ein-Komponentensystem (nach Askeland, 1996)

A: Die Temperatur der Schmelze liegt oberhalb der Solidustemperatur und die latente Wärme wird durch den Festkörper abgeführt, so können sich keine Erhebungen an der Wachstumsfront ausbilden. Die Kristallschicht wird aus polygonalen Kristallen gebildet.

B: Die Schmelze wird unterkühlt, so dass ihre Temperatur unterhalb der Solidustemperatur liegt. Erhebungen in der Front können zu Dendriten anwachsen. Es entsteht ein fest/flüssiges-Phasengemisch. (PDA: Primärer Dendriten Abstand) 


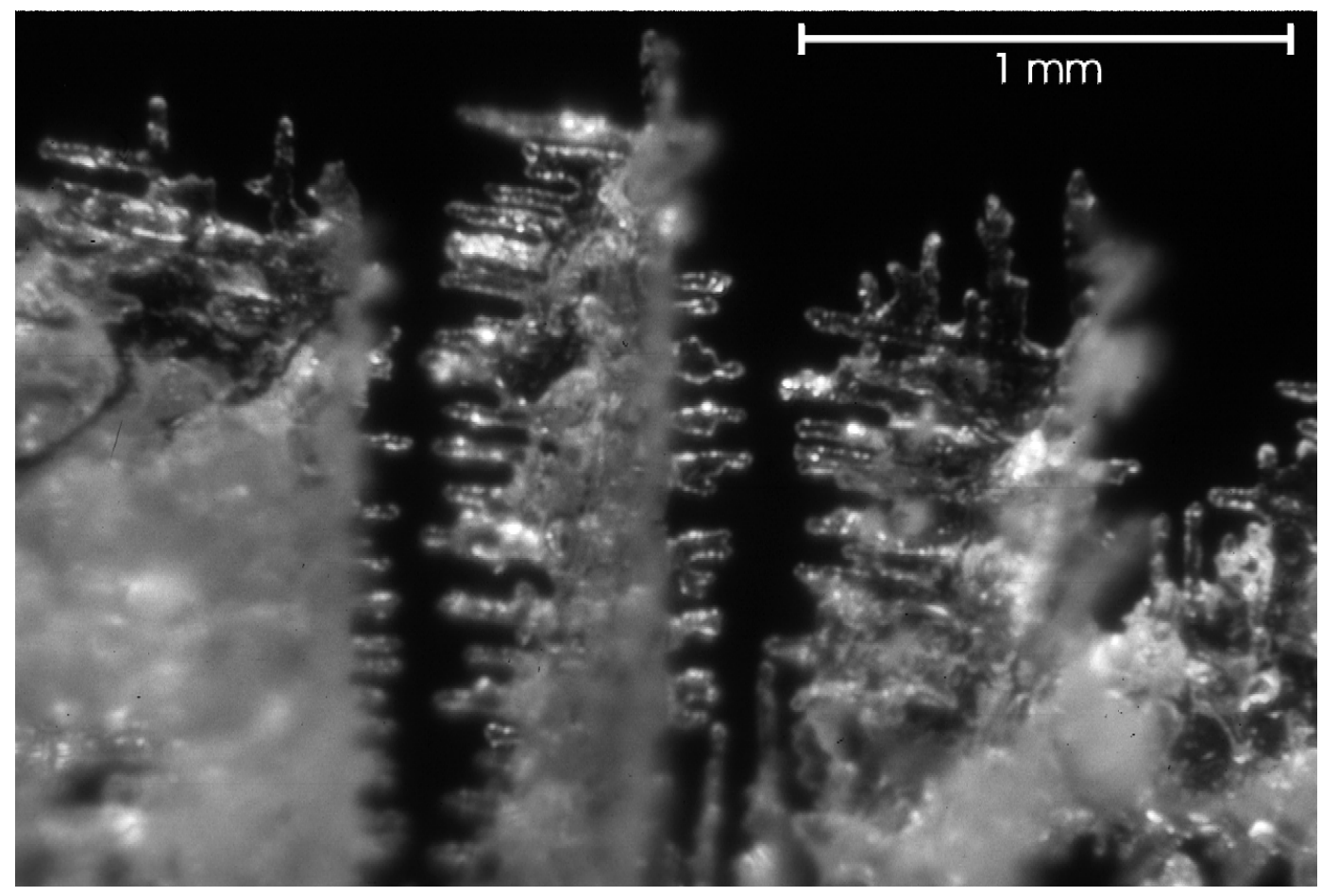

Abbildung 3.4: Nahaufnahme von Dendriten

Die primären Dendrite wachsen parallel nebeneinander mit einem typischen Abstand von $\approx 0,4 \mathrm{~mm} .\left(\Delta T=24,5 \mathrm{~K}, C_{o}=26\right.$ gew $\left.\%, \theta=0,5 \mathrm{~K}, \Omega=6,3 \frac{\mathrm{rad}}{\mathrm{s}}, E=1,3 \cdot 10^{-5}\right)$

\subsubsection{Kaminentstehung}

In metallischen Legierungen kann man in den dendritischen Gefügen oft Bereiche mit einer hoher Porosität („,freckles“) beobachten (z. B. Tewari \& Shah, 1992 und 1996). Diese Regionen erstrecken sich tief entlang der Erstarrungsrichtung, sind jedoch horizontal eng begrenzt. Sie haben den unerwünschten Effekt, dass sie die Festigkeit des Materials vermindern. Zur genaueren Untersuchung wurden Analogexperimente mit Ammoniumchlorid durchgeführt (McDonald \& Hunt, 1969 und 1970). Die „weichen“ Stellen werden hierbei mit Strömungen innerhalb der porösen Dendritenschicht in Zusammenhang gebracht, die zum Wiederaufschmelzen der Kristalle führen können. Copley et al. (1970) identifizieren die Strömungen als konzentrierte Aufströme (Plumes), die aus tiefen Kanälen (Kaminen) (Abb. 3.5) in der Schicht steigen (vgl. auch Abb. 3.6). In den Plumes steigt kaltes, leichtes Material mit einer geringeren Konzentration auf.

Der Mechanismus, der zur Ausbildung der Kamine führt, wird von Worster (1991) und Tait et al. (1992) durch konvektive Instabilitäten innerhalb der kristallinen Dendritenschicht erklärt. An den Kristallisationsfronten der Dendriten entsteht bei der Erstarrung in einer Grenzfläche Flüssigkeit, die mit der Komponente B der Lösung 


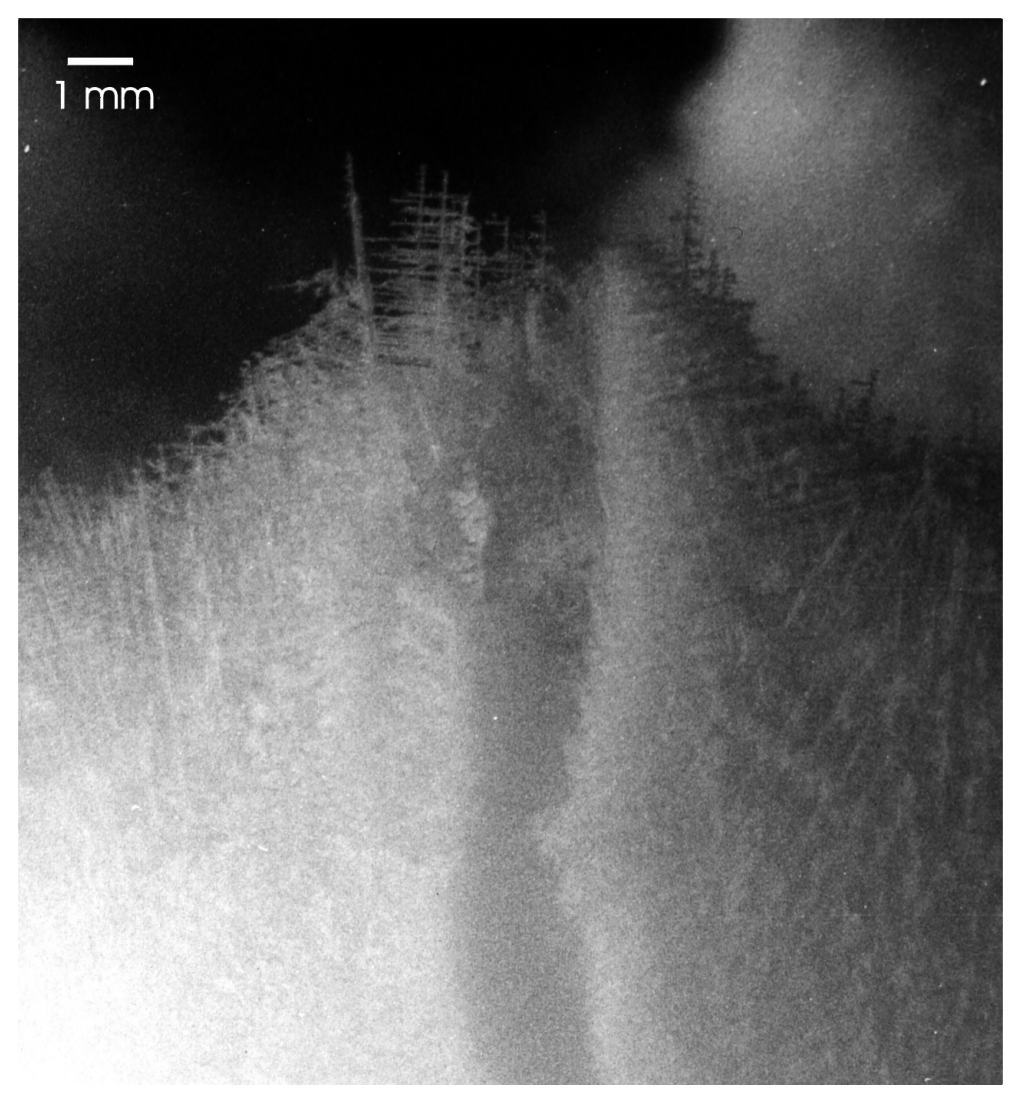

Abbildung 3.5: Kaminausgang

Kegelförmiger Ausgangsbereich eines Kamins mit deutlicher Dendritenstruktur der umgebenden Schicht. Der Kamindurchmesser beträgt $2 \mathrm{~mm} .\left(\Delta T=24,5 \mathrm{~K}, C_{o}=26 \mathrm{gew} \%\right.$, $\left.\theta=0,5 \mathrm{~K}, \Omega=2,1 \frac{\mathrm{rad}}{\mathrm{s}}, E=3,8 \cdot 10^{-5}\right)$.

angereichert ist. Ist dieses die leichtere Komponente, erhält die Lösung Auftrieb und steigt durch das Dendritennetzwerk der porösen Schicht empor. Das Material kommt dabei in wärmere Regionen, wobei es zwar seine Temperatur zum Teil an die Umgebung anpassen kann, jedoch nicht seine Konzentration ( $L e \approx 79$ ), so dass es im Vergleich zur Umgebung nicht gesättigt ist. Benachbarte Dendrite können wieder in Lösung gehen, wodurch sich die Porosität erhöht und die Darcy Reibung (Tait et al. 1992) abnimmt. Dies hat wiederum eine Zunahme der Strömung zur Folge. Das Material kann so auf seinem Weg nach oben einen dendritenfreien Kanal in die Schicht „brennen“.

Im Allgemeinen hat die Dendritenschicht an der Wachstumfront nur eine geringe Rauigkeit $(<1 \mathrm{~mm})$. Etwa 20 min nach Entstehung der Kamine im $\mathrm{NH}_{4} \mathrm{Cl}-\mathrm{H}_{2} \mathrm{O}-$ System bilden sich jedoch kegelförmige Erhebungen um die Kaminausgänge (Abb. 3.5 und Abb. 3.6). Sie entstehen durch den Austritt der um einige Grad kälteren Aufströme 
aus dem Kanal. Ihre Temperatur unterschreitet damit den Solidus der Umgebungsflüssigkeit, welches an den Plumerändern auskristallisiert.

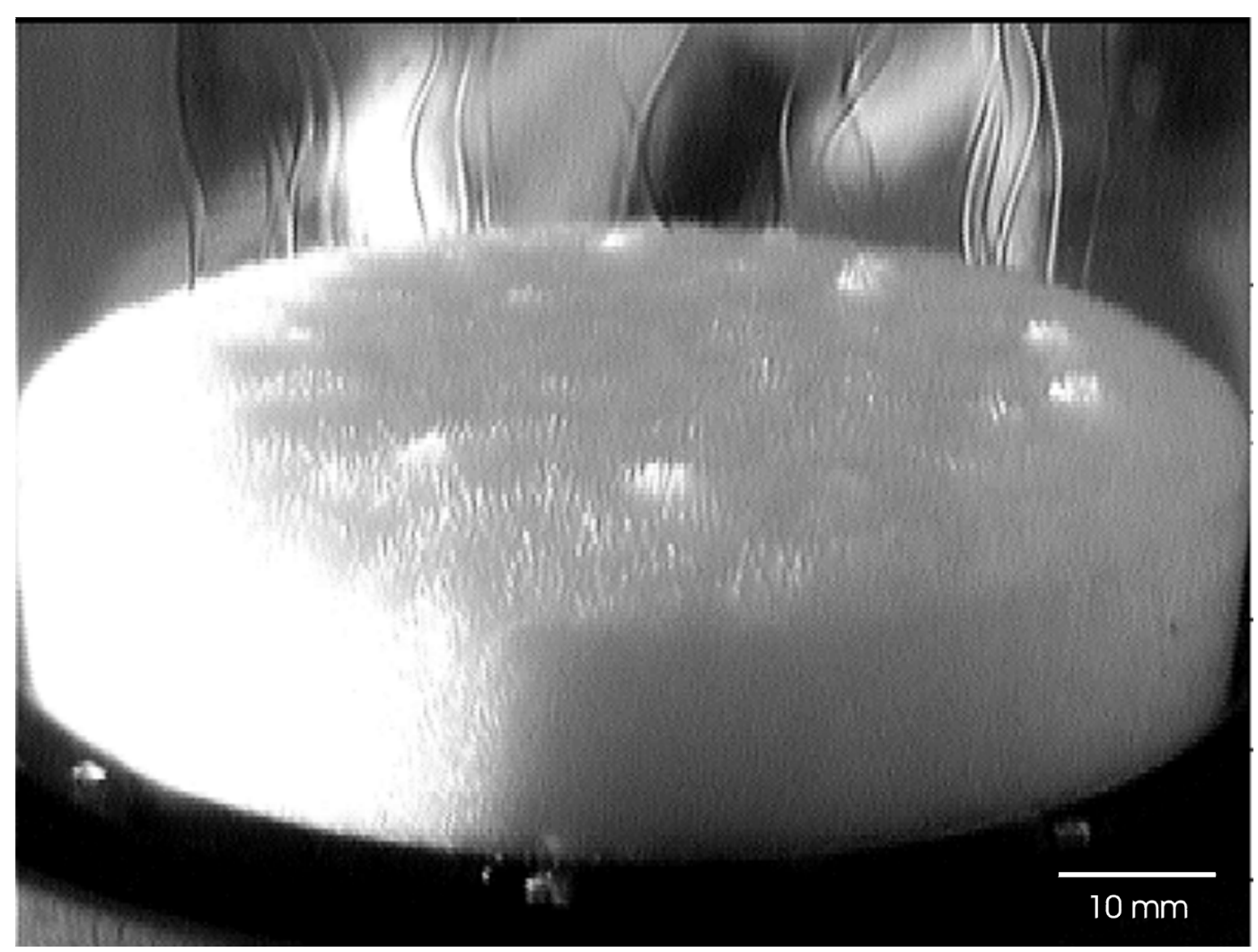

\section{Abbildung 3.6: Dendritenschicht mit Plumes}

Aus der im Allgemeinen ebenen Dendritenschicht ragen einige kegelförmige Schlote um ca. 2-3 mm heraus. In einigen Fällen sind die Plumes, die aus den Schloten aufsteigen, zu erkennen. Die Helixform der Plumeschläuche ist deutlich zu erkennen. $(\Delta T=36,5 \mathrm{~K}$, $\left.C_{o}=26 \mathrm{gew} \%, \theta=5,3 \mathrm{~K}, \Omega=0 \frac{\mathrm{rad}}{\mathrm{s}}\right)$.

\subsubsection{Konvektionsmoden}

Die lineare Stabilitätsanalyse der Konvektion in einer porösen Schicht (Worster, 1992) ergibt eine bi-modale Stabilitätskurve. Die erste instabile Mode bei kleinen Wellenlängen wird als „Grenzschicht-Mode“ bezeichnet. Die zweite Mode, die bei größeren Wellenlängen auftritt, wird „Dendritenschicht-Mode“ genannt. Die Bezeichnungen spiegeln die Konvektionsarten wider, die man in Laborexperimenten mit Ammoniumchlorid beobachten kann. 
Unmittelbar nach der ersten Keimbildung kommt es in der instabilen Grenzschicht an der Wachstumsfront zu einer kleinskaligen Strömung („Grenzschicht-Mode“). Die Auf- und Abströme haben die Form von dünnen Fäden („Finger“) und erstrecken sich nur über wenige Zentimeter oberhalb der Front. Der Fingerdurchmesser liegt etwa in der Größenordnung des primären Dendritenabstands. Diese Art der Konvektion wird als doppelt-diffusive Konvektion oder auf Grund ihres Aussehens als Fingerkonvektion bezeichnet.

Die zweite Konvektionsform ist die großskalige Plumekonvektion, die ihren Ursprung in den Kanälen innerhalb der porösen Dendritenschicht hat („,DendritenschichtMode“). Die Aufströme werden von engen, helikal aufsteigenden Flüssigkeitsschläuchen (Plumes) gebildet. Der Durchmesser eines Plumes bzw. eines Kamins ist mit typischerweise $2 \mathrm{~mm}$ etwa fünfmal größer als ein primärer Dendritenabstand. Die Plumes steigen hoch auf in die flüssige Phase oberhalb der Front und mischen sich auf ihrem Weg mit der Umgebungsflüssigkeit. Die diffusen Abströme dringen dann wieder in die poröse Kristallschicht ein. Deren Material ist an $\mathrm{NH}_{4} \mathrm{Cl}$ angereichert, wodurch die Kristallisation innerhalb der Schicht fortgesetzt und die Konvektion weiterhin in Gang gehalten wird.

Die kleinskalige Fingerkonvektion wird während des Erstarrungsvorgangs von der großskaligen Plumekonvektion abgelöst. Der Wechsel setzt ein, wenn die Kristallschicht eine kritische Höhe von etwa $10 \mathrm{~mm}$ überschritten hat, wobei die beiden Konvektionsarten für eine kurze Zeitspanne koexistieren.

Der Einsatz der Plumekonvektion kann, analog zum klassischen Rayleigh-Bènard Problem, über eine kritische Rayleighzahl $R_{m}$ beschrieben werden, die das Verhältnis der die Konvektion antreibenden zu den hemmenden Kräften beschreibt (Chen \& Chen, 1991 und Worster, 1991):

$$
R_{m}=\frac{\beta \Delta C g \Pi H}{\nu \kappa_{C}}
$$

g: Schwerebeschleunigung; H: Schichtdicke; $\kappa_{C}$ : chem. Diffusionskoeffizient; П: Permeabilität

Die Konvektion wird im vorliegenden System hauptsächlich durch die Konzentrationsdifferenz $\Delta C$ über die Schicht angetrieben. Da die Konvektion innerhalb eines porösen Mediums stattfindet, hemmt im Wesentlichen die Darcy Reibung die Strömung. Auf Grund der schwierigen Bestimmung der Schichtpermeabilität variiert der experimentell ermittelte Wert für $R_{m}$ bei Konvektionseinsatz zwischen 25 (Tait \& Jaupart, 1992) und 250 (Chen \& Chen, 1991 und Chen et al., 1994). 


\section{Kapitel 4}

\section{Versuchsaufbau}

Zur experimentellen Untersuchung des Kristallisationsprozesses eines binären Systems und dem daraus resultierenden Konvektionsprozess unter dem Einfluss von Corioliskräften wurde eine neue Versuchsanordnung entwickelt. Die Abmessungen des rotierbaren Teils der Anlage wurden dazu so gewählt, dass eine möglichst hohe Winkelgeschwindigkeit bzw. möglichst kleine Ekmanzahl erreicht werden kann, ohne einen dominanten Anteil an Zentrifugalkräften zu erhalten. Die Tankgröße wurde so optimiert, dass Ekmanzahlen bis $7,5 \cdot 10^{-6}$ realisiert werden können. Weiterhin wurde eine Visualisierung der Strömungsstrukturen aufgebaut.

\subsection{Rotationsapparatur}

Die Experimente sind, bis auf wenige Ausnahmen (s. Kap. 7.2), in einer Rotationsapparatur (Abb. 4.1) durchgeführt worden. Als Tankmaterial wurde transparentes PMMA (Polymethylmethacrylat, Plexiglas) gewählt, um die Beobachtung des Kristallwachstums und die Visualisierung der Strömung zu ermöglichen. Die Hauptkomponenten der Anlage sind drei voneinander unabhängige Kammern (1-3).

Im zentralen, rotierbaren Zylinder (4) befindet sich das Arbeitsmedium (2), eine wässrige Ammoniumchloridlösung $\left(\mathrm{NH}_{4} \mathrm{Cl}-\mathrm{H}_{2} \mathrm{O}\right)$. Der Zylinder hat einen Innendurchmesser von $80 \mathrm{~mm}$ und eine zu befüllende Höhe von $150 \mathrm{~mm}$. Die Wandstärke beträgt $5 \mathrm{~mm}$. An seiner Außenseite befindet sich eine Millimeter-Skala zur direkten Messung der Kristallschichthöhe. Der Zylinder wird oben mit einem Plexiglasdeckel (6) verschlossen. In der Mitte des Deckels befindet sich ein $90 \mathrm{~mm}$ langes Plexiglasrohr (7) mit einem Innendurchmesser von $11 \mathrm{~mm}$. Durch dieses Rohr können während des 
Experiments Temperaturmessungen innerhalb des Zylinders durchgeführt oder Proben des Fluids entnommen werden.

Der Boden besteht aus einer 10 mm starken Messingplatte (5), die eine gute Wärmeleitfähigkeit gewährleistet. In der Mitte der Platte, ca. $2 \mathrm{~mm}$ unterhalb der Oberfläche, ist ein Thermoelement巴 (9) eingelassen, über das die Plattentemperatur auf $\pm 0,2 \mathrm{~K}$ genau bestimmt werden kann. Die Lösung reagiert sehr heftig mit den meisten Metallen und Metalllegierungen. Deshalb ist die Bodenplatte mit einer dünnen Lackschicht versiegelt worden.

Die Salzlösung wird von unten durch ein Temperaturbad (1) gekühlt. Dieses wird über einen Kryostaten gespeist, der die eingestellte Temperatur auf $\pm 0,2 \mathrm{~K}$ einhält. Nachdem das Bad seine Solltemperatur $T_{1}$ erreicht hat, beträgt die maximale Temperaturdifferenz zwischen der Flüssigkeit im Kryostat und der im Temperaturbad nur 0,5 K. Als Kühlflüssigkeit wird eine 50 \%-ige wässrige Ethylenglykollösung verwendet.

Die oben beschriebene Anordnung wird von einem quadratischen Plexiglastank (8) umschlossen. Dieser stellt ein Temperaturbad (3) zur genauen Kontrolle und Regulierung der Außentemperatur dar. Bei der benutzten Visualisierungsmethode (Schattenverfahren) würden durch den Zylinder starke Linseneffekte auftreten. Diese können mit Hilfe des quadratischen Tanks zum größten Teil korrigiert werden, da die Brechungsindizes der drei Medien sehr ähnlich sind ( $n_{D}=1,382\left(26\right.$ gew\% ) 2, $n_{D}=1,491$ (PMMA), $n_{D}=1,39$ (50\%-ige Ethyl.-Lösung)).

Das Bad wird von einem zweiten, externen Wärmebad gespeist. Die Temperatur $T_{3}$ im äußeren Kasten wird über ein Thermoelement (10) kontrolliert. Das externe Kryostatenbad benötigt etwa 5 min, um sich nach einer Änderung der Solltemperatur von $3 \mathrm{~K}$ neu einzustellen. Diese Zeitdifferenz muss für die Regelung bedacht werden.

Die Bäder 1 und 3 haben jeweils zwei $\mathrm{Zu}-$ und Abflüsse in den Bodenflächen (Abb. 4.2). Sie sind diagonal zueinander in den Tankecken angeordnet, so dass eine gute Durchmischung der einfliessenden Flüssigkeit gewährleistet ist. Im Deckel und im Boden des äußeren Tanks sowie zwischen Zylinderboden und Kühlbad (1) verhindern Lippendichtringe (15) das Austreten von Flüssigkeit.

Der Zylinder ist über eine Hohlachse (11) drehbar gelagert. Der Antrieb erfolgt über einen computergesteuerten Schrittmotor (12). Die Drehzahl $\omega$ kann in den durchgeführten Experimenten in einem Bereich von 5-100 $\pm 0,2 \frac{U}{\min }$ variiert werden. Bei einer Dreh-

\footnotetext{
${ }^{1}$ Die genauen Spezifikationen der verwendeten Messgeräte sind in Anhang A beschrieben.

${ }^{2} \mathrm{Sämtliche}$ Angaben der Konzentration in dieser Arbeit beziehen sich auf gew\% von $\mathrm{NH}_{4} \mathrm{Cl}$ in $\mathrm{H}_{2} \mathrm{O}$
} 


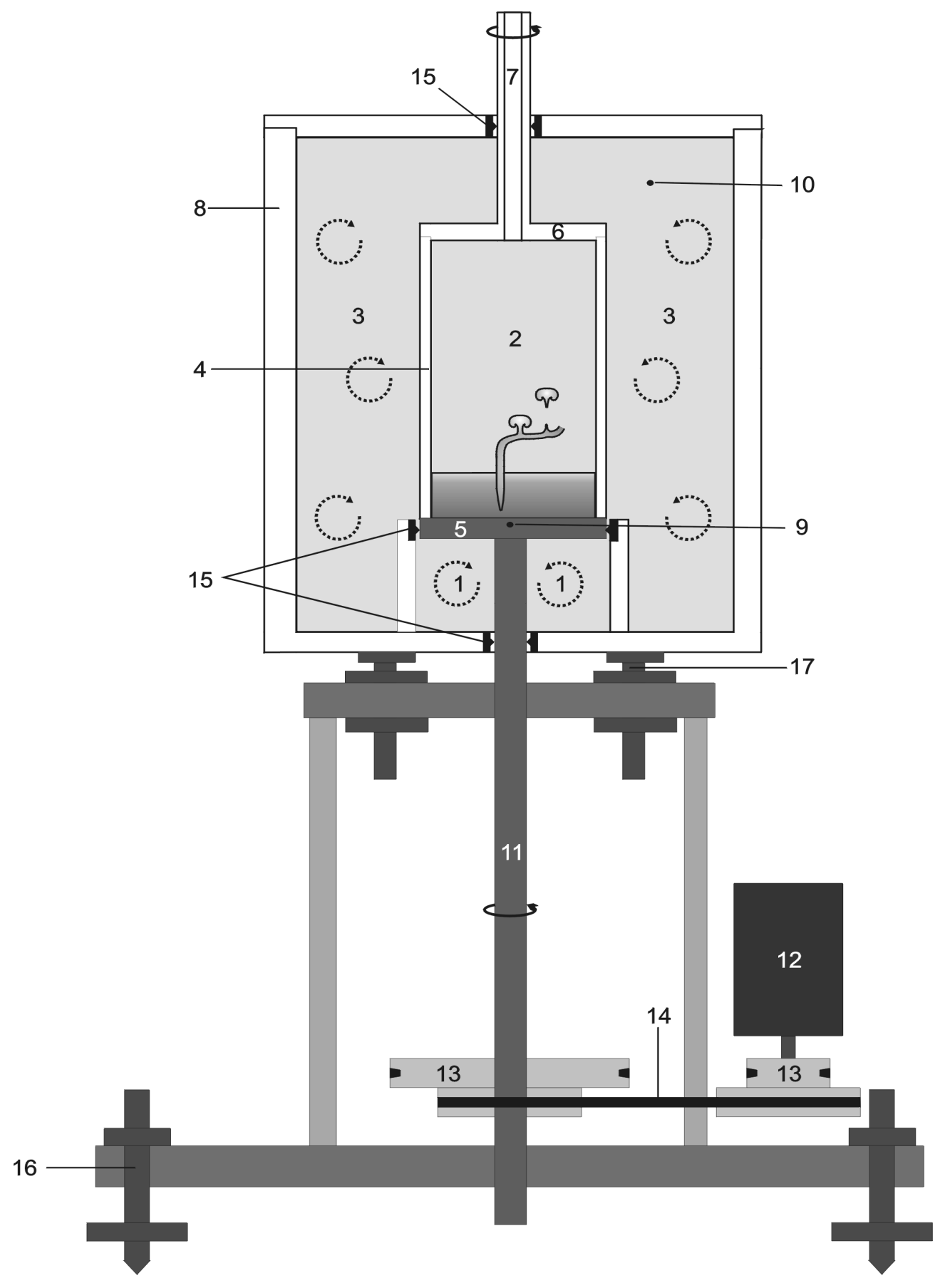

\section{Abbildung 4.1: Versuchsaufbau}

1: unteres Kühlbad, 2: wässrige Ammoniumchlorid-Lösung als Arbeitsmedium, 3: äußeres Temperaturbad, 4: rotierbarer Plexiglaszylinder, 5: Bodenplatte (Messing), 6: Zylinderdeckel (PMMA), 7: Plexiglasrohr, 8: quadratischer Tank (PMMA), 9, 10: Thermoelemente, 11: Drehachse, 12: Schrittmotor, 13 Antriebscheiben, 14: Keilriemen, 15: Dichtungslippen, 16, 17: Justierschrauben 


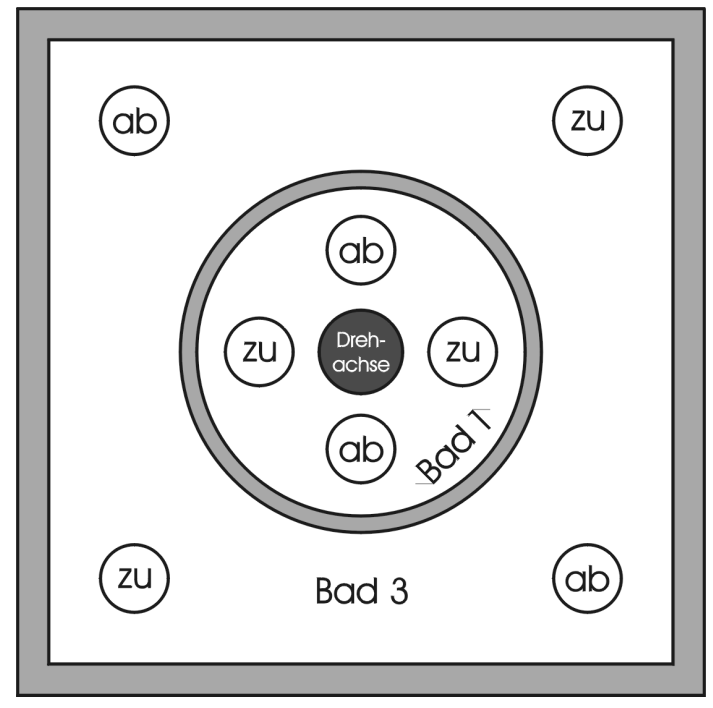

\begin{abstract}
Abbildung 4.2: Kühlbäder
Blick von unten auf die Anschlüsse der Temperaturbäder. Die Bäder verfügen über jeweils zwei Zu-und Abflüsse, die einander diagonal gegenüber liegen.
\end{abstract}

zahl von $\omega=100 \frac{U}{\min }$ bzw. einer Winkelgeschwindigkeit von $\Omega=10,47 \frac{\mathrm{rad}}{\mathrm{s}}$ beträgt die maximale Zentrifugalbeschleunigung

$$
a=\Omega^{2} r=4,39 \frac{m}{s^{2}} \approx \frac{1}{2} \cdot g
$$

mit dem Radius $r=0,04 \mathrm{~m}$ des Zylinders und der Schwerebeschleunigung $g=9,81 \frac{\mathrm{m}}{\mathrm{s}^{2}}$. Demgegenüber ist die zu erwartende Coriolisbeschleunigung $a=2 \Omega v$ um beinahe zwei Größenordnungen kleiner als die Schwerebeschleunigung, da mit einer mittleren Strömungsgeschwindigkeit $v$ von $10 \frac{\mathrm{mm}}{\mathrm{s}}$ gerechnet wird. Die Schwere ist somit bei der verwendeten Maximalgeschwindigkeit immer noch die dominierende Kraft.

An der Achse sind zwei Riemenscheiben (13) mit einer Übersetzung von 1:1 und 1:5 angebracht. In den Experimenten mit geringen Drehzahlen $\left(5-30 \frac{U}{\min }\right)$ ist die große Übersetzung notwendig, um den Motor im optimalen Arbeitsbereich laufen zu lassen. Der Motor ist auf der untersten Plattform der Apparatur angebracht. Um die Schwingungen des Motors vom Aufbau zu entkoppeln, wird er mit Gummipuffern befestigt. Schwingungsübertragungen durch den Keilriemen (14) können vernachlässigt werden.

Der gesamte Aufbau kann mit Hilfe von drei Justierschrauben (16) horizontal ausgerichtet werden. Zudem ist es möglich, mit vier weiteren Schrauben (17) den oberen quadratischen Tank relativ zum inneren Zylinder in seiner Höhe zu variieren. Mit dieser Vorrichtung wird die Lauffläche an der Bodenplatte genau auf die Dichtlippe ausgerichtet, so dass die Reibung zwischen ihnen minimiert und die Dichtfunktion optimiert wird. 


\subsection{Visualisierung der Strömung}

Zur Untersuchung der Strömungsstruktur wird das Schattenverfahren als Visualisierungsverfahren benutzt. Dieses Verfahren nutzt die Lichtbrechung an Temperatur- und Konzentrationsunterschieden in einem transparenten, inhomogenen Medium aus. Beim Schattenverfahren entsteht der Kontrast durch die Ablenkung eines Anteils des Lichtstrahles an den Inhomogenitäten und der daraus folgenden Reduktion der Intensität des direkten Strahles (Hellfeld-Aufnahme). Die einfachste Methode ist das zentral projizierte Schattenverfahren (Oertel \& Oertel, 1989). Hier genügen eine Punktlichtquelle und ein Schirm zur Visualisierung der Ablenkung.

Abbildung 4.3 skizziert den optischen Aufbau. Von einer Lichtquelle ausgehend laufen die Strahlen durch den Versuchstank und treffen auf eine Mattscheibe. Diese würde gleichmäßig ausgeleuchtet, wäre der Tank mit einer homogenen Flüssigkeit gefüllt. Passieren die Lichtstrahlen auf ihrem Weg jedoch einen Dichtegradienten, werden sie dort so gebrochen, dass auf dem Schirm ein unbeleuchteter Bereich erscheint, ein Schatten. Auf die Mattscheibe wird also eine zweidimensionale Abbildung aller dreidimensionalen Dichte-Inhomogenitäten in der Flüssigkeit projiziert.

Zur Dokumentation und Auswertung der Strömungsstrukturen werden die Schattenbilder auf der Mattscheibe fotografiert. In den meisten Fällen reicht dazu eine Fotokamera aus. Bei sehr schnellen Bewegungen (hohe Winkelgeschwindigkeiten), oder wenn man Informationen über die zeitliche Entwicklung der Strömungsstruktur an einer Position innerhalb des Zylinders erhalten möchte, wird eine CCD-Kamera eingesetzt. Dazu ist die Synchronisation der Kamera mit der Rotationsbewegung des Zylinders notwendig. Erreicht wird dies durch einen Infrarotsensor, der an der Motorantriebsscheibe angebracht ist. Er sendet bei jeder Umdrehung ein Signal an die Kamera (Abb. 4.3), diese nimmt daraufhin ein Bild auf, das über eine Framegrabberkarte an einen PC geschickt wird. Die Karte gibt anschließend das Signal (Restart) an die Kamera zurück, dass die Aufnahme beendet ist, so dass diese ein neues Bild aufnehmen kann. Eine spezielle Software speichert automatisch die einzelnen Bilder zu einem digitalen Video ab. So können bei Versuchen mit Rotation Bilderserien der zeitlichen Strömungsentwicklung gemacht werden.

Die Monochrom-Kamera arbeitet nach dem Progressive Scan (bzw. non-interlace) Prinzip. Sie liest den CCD-Chip in einem Durchgang vollständig aus, während Standard-Kameras gemäß der Videonorm die geraden und ungeraden Zeilen in zwei Durchgängen auslesen (interlace). Bei schnellen Vorgängen, wie den hier untersuchten, 


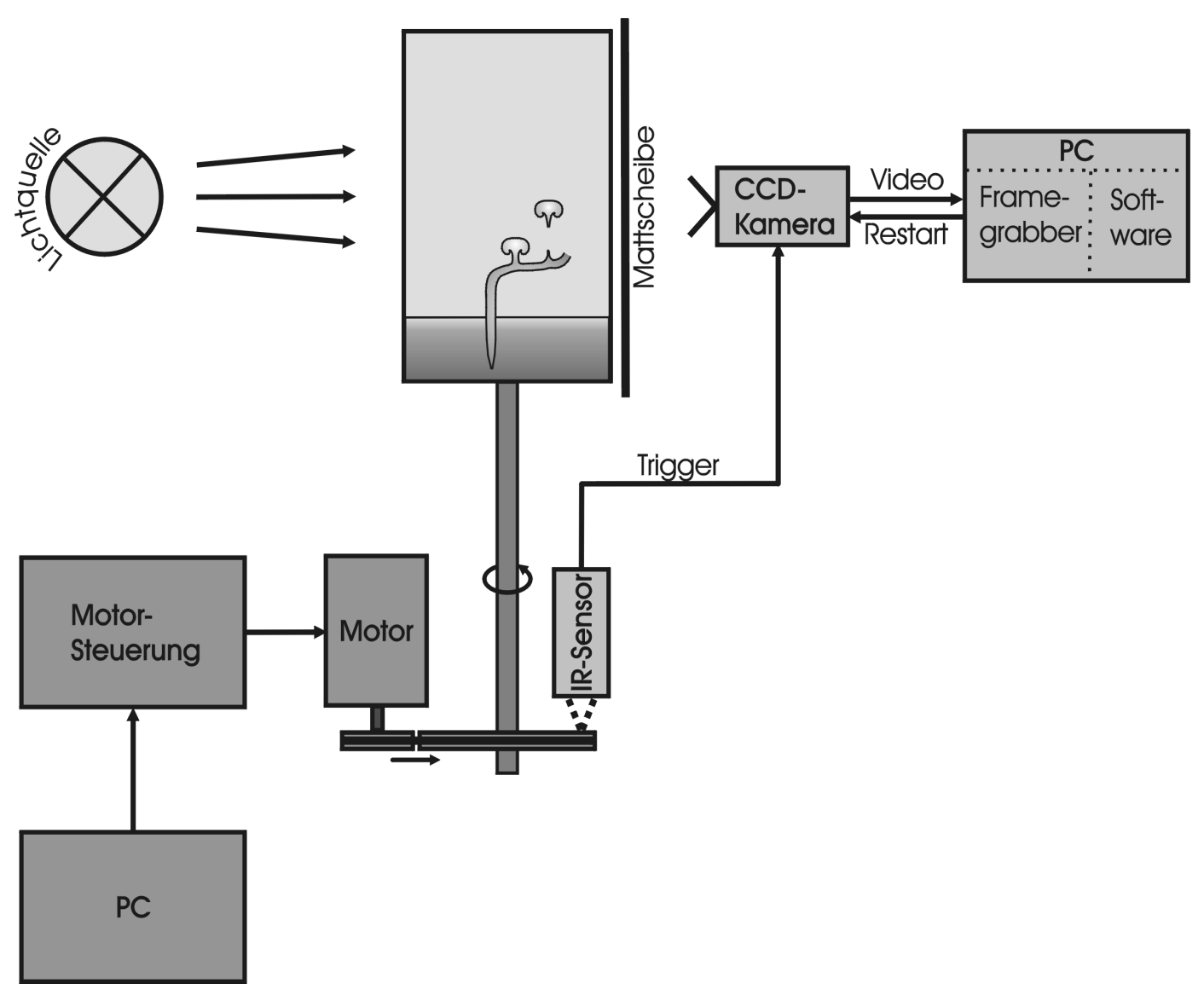

Abbildung 4.3: Optischer Aufbau

Die Lichtstrahlen gehen von einer Lichtquelle aus, laufen durch den rotierenden Zylinder und treffen auf eine Mattscheibe. Ein Fotoapparat bzw. eine Videokamera (CCDKamera) nehmen die Schattenbilder von der Scheibe auf. Der IR-Sensor (Lichtschranke) sendet pro Umdrehung ein Aufnahmesignal an die CCD-Kamera, woraufhin diese eine Bildsequenz aufnimmt. Das Videosignal geht über eine Framegrabber-Karte an den PC und wird dort weiterverarbeitet. Der Zylinder wird von einem computergesteuerten Schrittmotor angetrieben. 
ist dieser Vorgang sehr unvorteilhaft. Das abzubildende Objekt bzw. die Strömungsstruktur hätte sich in der Zeit, die der Rechner zum Auslesen des ersten Halbbildes benötigt, bereits wieder verändert, und man erhielte ein unscharfes Bild. Beim verwendeten non-interlace Verfahren entfällt dieser Nachteil. 


\section{Kapitel 5}

\section{Versuchsablauf}

\subsection{Vorbereitungen zu einem Experiment}

Die wässrige Ammoniumchlorid-Lösung $\left(\mathrm{NH}_{4} \mathrm{Cl}-\mathrm{H}_{2} \mathrm{O}\right)$ mit der gewünschten Konzentration $C_{\circ}$ wird zu Beginn des Experiments in den Zylinder gegeben. Sie kann mit einer Genauigkeit $\square$ von $3 \cdot 10^{-3}$ gew\% hergestellt werden. Anschließend werden Luftblasen, die sich an den Wänden und am Boden gebildet haben, vorsichtig entfernt, da sie andernfalls später bei der Visualisierung stören würden. Auf dem Zylinder wird der Deckel angebracht und festgeschraubt. Dann können die Temperaturbäder befüllt werden. Die Temperatur der Bäder sollte dabei den Liquidus der aktuellen Konzentration nicht unterschreiten. Auch hier müssen alle Luftblasen entfernt werden. Abschließend wird der Deckel des äußeren Kastens geschlossen.

Die Temperatur im $T_{3}$ äußeren Bad wird bis auf $0,5 \mathrm{~K}$ auf die Liquidustemperatur $T_{L}\left(C_{\circ}\right)$ eingestellt. Dann wird das Temperaturgleichgewicht zwischen dem äußeren Bad mit $T_{3}$ und der Lösung im Zylinder mit $T_{2}$ abgewartet. $\theta$ ist die Differenz zwischen $T_{L}\left(C_{0}\right)$ und $T_{2}$ nach Erreichen des thermischen Gleichgewichtes. Zur Einstellung des thermischen Gleichgewichtes muss die thermische Diffusionszeit $t_{D}$ abgewartet werden. Sie beschreibt die Zeitspanne, in der ein Temperaturgradienten allein durch Wärmeleitung über eine Distanz $L$ abgebaut wird:

$$
t_{D}=\frac{L^{2}}{\kappa_{T}}
$$

Für den Radius des Zylinders $(L=4,5 \mathrm{~cm})$ und den thermischen Diffusionskoeffizienten der $\mathrm{NH}_{4} \mathrm{Cl}$-Lösung $\kappa_{T}=1,336 \cdot 10^{-3} \frac{\mathrm{cm}^{2}}{\mathrm{~s}}$ beträgt $t_{D}$ etwa 4,2 Stunden. Die thermische

\footnotetext{
${ }^{1}$ Meßgenauigkeit der Waage: 0,01 g, Abwiegefehler: 0,02 g
} 
Trägheit² für die Plexiglaswände ist dagegen mit ca. 3,8 min vernachlässigbar. Man kann also davon ausgehen, dass nach einer Wartezeit von 4,5 Stunden ein thermisches Gleichgewicht zwischen dem äußeren Bad und der Flüssigkeit im Zylinder erreicht wird. In dieser Zeit wird der Kryostat für die Kühlung des unteren Bades auf die gewünschte Abkühltemperatur $T_{1}$ vorgekühlt. Die Unterkühlung $\Delta T$ ergibt sich aus der Differenz zwischen $T_{L}\left(C_{0}\right)$ und $T_{1}$.

Das stationäre Anfangsstadium für ein Experiment bzw. ein thermisches Gleichgewicht wird als erreicht angesehen, wenn die Temperaturen in den drei Kammern bis auf $\pm 0,5 \mathrm{~K}$ übereinstimmen $\left(T_{1}=T_{2}=T_{3}=T_{L}\left(C_{o}\right)\right)$. Bei Experimenten unter dem Einfluss von Corioliskräften wird der Zylinder mindestens eine Stunde vor dem Start des Experiments mit einer konstanten Winkelgeschwindigkeit $\Omega$ gedreht. Damit hat man als Anfangsbedingung einen rotierenden starren Körper, d. h. in der Flüssigkeit sind keine Scherschichten mehr vorhanden.

\subsection{Ablauf eines Experiments}

Das Experiment startet, wenn der Kühlkreislauf geöffnet wird. In Abbildung 5.1 erkennt man, dass die Kühlplattentemperatur $T_{1}$ nach dem Start sehr schnell abfällt. Nach typischerweise 10 min stellt sich die gewünschte Solltemperatur ein. Sie bleibt während des Experiments bis auf $\pm 0,4 \mathrm{~K}$ konstant.

Während des Experiments werden die Kristallschichthöhe $H$, die Bodentemperatur der Kühlplatte $T_{1}$, die Temperatur in der flüssigen Phase $T_{2}$ und die Temperatur im äußeren Temperaturbad $T_{3}$ gemessen. In Rotationsexperimenten kann die Bodentemperatur während des Experimentes nicht gemessen werden, hier werden Werte aus Vergleichsexperimenten ohne Rotation herangezogen. Da der zeitliche Verlauf der Bodentemperatur $T_{1}$ für gleiche Kühltemperaturen in verschiedenen Experimenten ohne Rotation gut übereinstimmt, scheint dieses Vorgehen gerechtfertigt. Das Kristallwachstum und die Entwicklung der Konvektion werden mit Videoaufnahmen und Fotografien dokumentiert.

$T_{3}$ wird ständig mit $T_{2}$ verglichen und nachgeregelt. Dabei muß die Temperatur im äußeren Bad der Temperatur innerhalb des Zylinders stets um 0,5 bis 1,0 ${ }^{\circ} \mathrm{C}$ vorauseilen (Abb. 5.2). Das ist notwendig, um die Zeitspanne zu kompensieren, die das Temperaturbad benötigt, um sich auf eine neue Solltemperatur einzustellen. Ein Indiz für

\footnotetext{
${ }^{2}$ Plexiglas: $\kappa_{T}=1,09 \cdot 10^{-3} \frac{\mathrm{cm}^{2}}{\mathrm{~s}}, L=5 \mathrm{~mm}$
} 


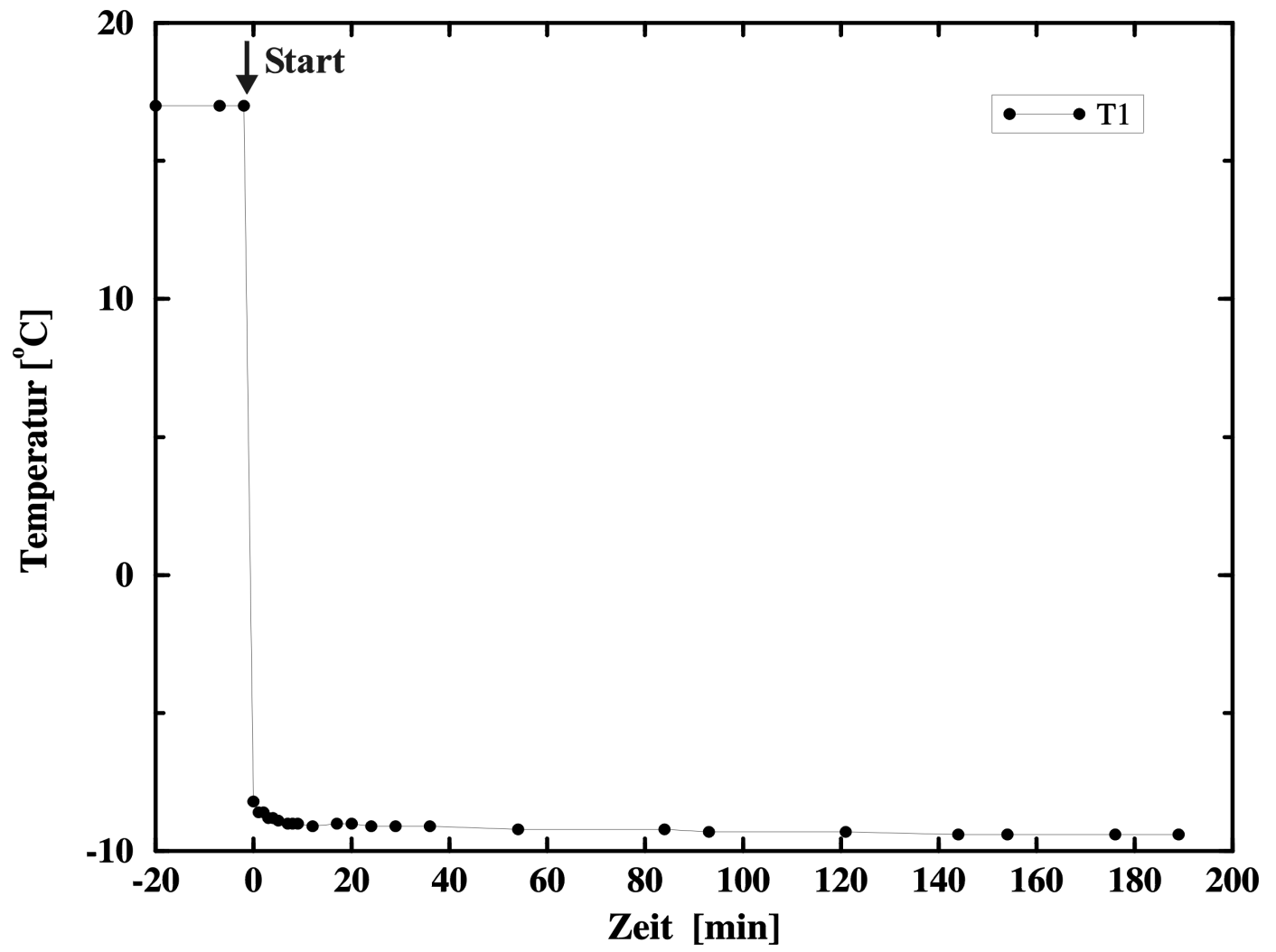

\section{Abbildung 5.1: Bodentemperatur}

Abfall der Bodentemperatur $T_{1}$ während eines Experiments nach dem Öffnen (Start) des Kühlkreislaufs. Die gewünschte Solltemperatur zur Kühlung wird nach etwa 10 min erreicht.

eine ausreichende Regelung der Außentemperatur ist das Kristallisationsverhalten am inneren Rand der Zylinderwand. Wäre $T_{3}$ zu niedrig eingestellt, käme es zu verstärktem Wachstum. Bei einer zu hoch eingestellten Temperatur würde das Wachstum am Rand verringert. Bei einer unkorrekten Regelung würde es dort in extremen Fällen zu vollständigem Kristallisationsstillstand kommen. Das Wachstum beschränkte sich dann auf den inneren Bereich des Zylinders und ein „Kristallberg“ würde entsteht. In den durchgeführten Experimenten mit Temperaturnachregelung findet das Kristallwachstum homogen verteilt über die gesamte Fläche der Bodenplatte statt.

Im größten Teil der Experimente wird die Temperatur von Hand nachgeführt. In den späteren Versuchen wird ein Temperaturbad mit externem Temperaturfühler benutzt, über den die Regelung automatisch erfolgt. Der externe Fühler wird dabei durch das Rohr im Deckel eingeführt und muss für eine optimale Temperaturregelung darin verbleiben. Für Rotationsexperimente ist das nicht möglich, da der Fühler, der nicht genau mittig untergebracht werden kann, starke Wirbel in der Flüssigkeit verursacht. In diesen Fällen wird die Temperatur auch weiterhin manuell geregelt. 


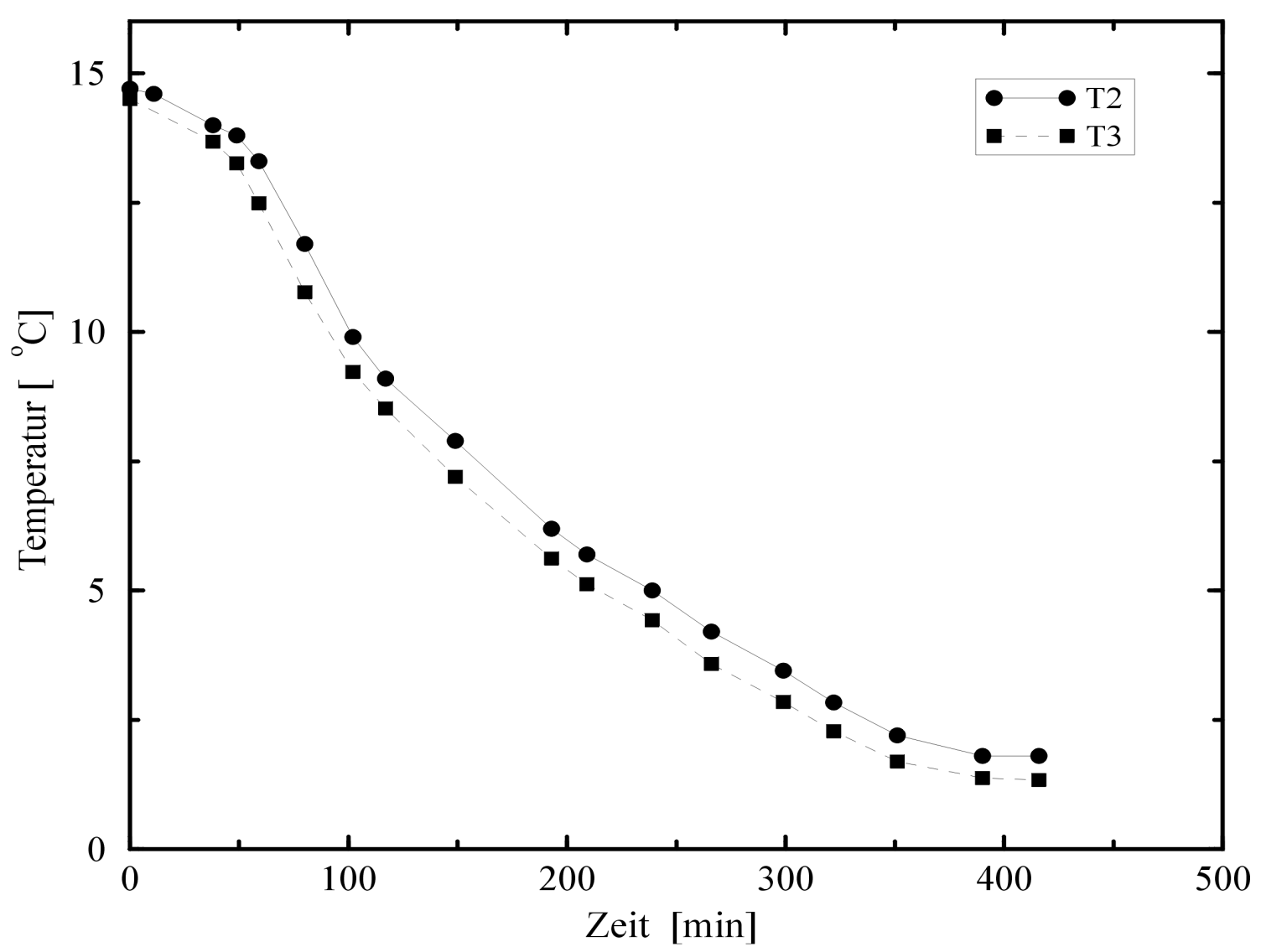

Abbildung 5.2: Regulierung der äußeren Wasserbadtemperatur

Zeitliche Entwicklung der Temperatur innerhalb des Zylinders $T_{2}$ und der Temperatur im äußeren Temperaturbad $T_{3}$ während eines Experimentes. $T_{3}$ liegt um etwa $0,5 \mathrm{~K}$ unterhalb von $T_{2}$. 


\section{Kapitel 6}

\section{Messgrößen}

Im Folgenden werden die verwendeten Messverfahren vorgestellt und die Messgenauigkeiten der einzelnen Werte diskutiert.

\subsection{Höhe der porösen Dendritenschicht}

Die Höhe $H$ der porösen Dendritenschicht wird während des Experimentes direkt anhand eines Maßstabes gemessen, der an der Außenseite des Zylinders angebracht ist. Abhängig von der Geschwindigkeit der Kristallisation auf Grund der Kühltemperatur und des Experimentierverlaufs wird $H$ in Intervallen von 2 min bis 30 min gemessen. Bei Rotationsexperimenten mit hohen Rotationsraten wird $H$ über den Schattenriss z. T. während des Experimentes und anhand der Schattenbildaufnahmen nach dem Experiment bestimmt.

Durch die Verwendung des zentralprojizierten Schattenverfahrens kommt es zu einer Aufweitung des Lichtstrahls. Sie beträgt maximal 0,3 mm und liegt damit unterhalb der Messgenauigkeit. Die Schichthöhe ist jedoch zu keinem Zeitpunkt überall konstant. Vor allem im Anfangsstadium der Kristallisation, bevor sich eine homogene Schicht ausgebildet hat und ab dem Einsatz der Plumekonvektion, kommt es lokal zu Höhendifferenzen von bis zu $3 \mathrm{~mm}$, in extremen Fällen von bis zu $5 \mathrm{~mm}$. Die Höhe wird dann über die gesamte Fläche gemittelt. Man erreicht insgesamt eine Genauigkeit von $\pm 2 \mathrm{~mm}$. Ist die Schicht plan und homogen, beobachtet man Variationen in der Höhen von nur noch $1 \mathrm{~mm}$. 


\subsection{Plume-Anzahl und -Durchmesser}

Die Anzahl der sichtbaren Kamine in der Kristallschicht sowie die Anzahl der Plumes im Schattenbild wird in den gleichen Zeitintervallen notiert, in denen auch die Schichthöhe bestimmt wird.

In den Bildern wird ebenfalls durch den scharfe Kontrast zwischen Plume und Umgebungsflüssigkeit der Durchmesser der Plumestämme bestimmt. Die Aufnahmen können soweit vergrößert werden, dass eine Auflösung von 0,2 mm erreicht werden kann.

Die Schattenprojektion zeigt hauptsächlich den Konzentrationsgradienten zwischen Plume und Flüssigkeit, da der Plume sich zum einen thermisch schnell an seine Umgebung anpasst $\left(t_{D}=7,5 \mathrm{~s}\right.$, vgl. Kap. 5.1) und zum anderen der thermische Einfluss auf den Brechungsindex mit einem Brechungsindexkoeffizienten $n_{T}=1,7 \cdot 10^{-4} \mathrm{~K}^{-1} \mathrm{um}$ eine Größenordnung kleiner ist als der chemische Einfluss mit $n_{C}=1,8 \cdot 10^{-3}$ gew\% ${ }^{-1}$. Das Geschwindigkeitsprofil des Plumes dürfte jedoch über die scharfe Begrenzung hinausreichen. Dieser Unterschied wird in Kapitel 8.3 noch genauer diskutiert.

\subsection{Dendritengröße und Morphologie}

Die Anzahl der Kamine in der Dendritenschicht wird zum Teil während des Experiments oder anhand der aufgenommenen Bilder bestimmt. Der Durchmesser der Kaminausgänge an der Oberfläche und ihr Abstand zueinander können aus den Aufnahmen mit einer Genauigkeit von $0,5 \mathrm{~mm}$ bestimmt werden.

Die innere Struktur der Dendritenschicht wird ebenfalls für einige Experimente untersucht. Dazu wird nach Abschluss der Experimente die Dendritenschicht vorsichtig vom Boden gelöst und komplett aus dem Gefäß entfernt. Die Schichten werden dann in etwa 2-3 mm dicke Scheiben zerteilt. Geringere Scheibenmächtigkeiten sind mechanisch nicht stabil genug. Die Morphologie wird unter dem Mikroskop mit einer Auflösung von 0,1 mm oder mit der CCD-Kamera genauer untersucht.

\subsection{Strömungsgeschwindigkeit}

Die Messung der Strömungsgeschwindigkeit erfolgt über verschiedene Verfahren. Die Geschwindigkeit der Finger während der doppelt-diffusiven Konvektion und die der Tröpfcheninstabilität wird in den Videoaufnahmen der Schattenbilder bestimmt. 
Schwieriger ist die Ermittelung der Aufstiegsgeschwindigkeit der Plumes, da sie keine charakteristischen Punkte aufweisen, die sich zur Messung verfolgen lassen. Zudem schlägt ein Plume ab einer Höhe von wenigen Zentimetern einen dreidimensionalen Weg ein. Plumes und Finger führen jedoch öfter Dendritenfragmente aus der Kristallschicht mit sich, die als Tracerpartikel verwendet werden. Diese können in erster Näherung als kugelförmig angenommen werden. Festes Ammoniumchlorid hat zwar eine um etwa $35 \%$ höhere Dichte als die Lösung, der Anteil an festem Material in dem Partikel ist jedoch so gering (Hellawell et al., 1993), dass sich das Teilchen passiv mit der Strömung bewegt. Die Geschwindigkeit lässt sich mit einer Messgenauigkeit von $0,5 \mathrm{~mm} / \mathrm{s}$ über die Tracerpartikeln bestimmen.

\subsection{Konzentrationsmessung}

Die Schichthöhe allein ist nur ein unzureichendes Maß zur Bestimmung der Menge an auskristallisiertem Material. Die Kristallisation findet nicht nur an der oberen Grenzfläche zwischen der porösen Schicht und der flüssigen Phase statt, sondern auch innerhalb des Dendritennetzwerkes. Auch wenn die Schicht eine konstante Mächtigkeit erreicht hat, schreitet die Plumekonvektion weiter fort (siehe Abb. 7.8), d. h. es wird weiterhin Material mit geringerer Dichte nach oben transportiert. Dies bedeutet gleichzeitig, dass die Porosität abnimmt und der Anteil $\phi$ an festem Material in der Schicht im Laufe des Experiments zunimmt.

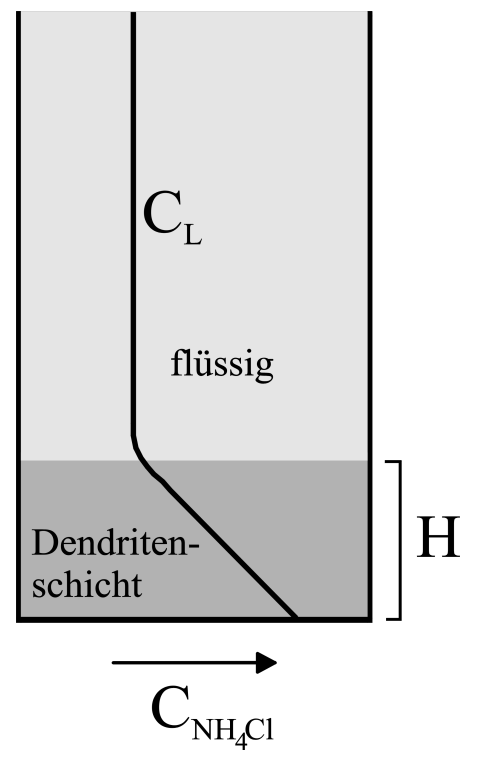
Abbildung 6.1: Konzentrationsprofil
Die mittlere Konzentration $\mathrm{C}$ von $\mathrm{NH}_{4} \mathrm{Cl}$
steigt linear innerhalb der porösen Schicht
an (vgl. Abb. 3.1). Bis auf einen kleinen
Übergangsbereich an der oberen Kristalli- sationsfront (poröse Schicht-Lösung) ist die Konzentration $C_{L}$ in der Flüssigkeit konstant. 
Die Bestimmung der Porosität $\chi$ (bzw. des Anteils der festen Phase $\phi=1-\chi$ ) während des Versuchs, beispielsweise durch Messung des typischen Dendritenabstands in unterschiedlichen Höhen zu verschiedenen Zeitpunkten, ist ohne Zerstörung des sensiblen Netzwerks nicht durchführbar. Chen \& Chen (1991) bestimmen die Porosität mit Hilfe von Computer-Tomografie. Diese Methode ist jedoch sehr aufwendig. Eine einfachere Möglichkeit der in situ Messung wird von Shirtcliffe et al. (1991) vorgestellt. Sie bestimmen den prozentualen Anteil $\phi$ an Kristallen während des Kristallisationsprozesses in einer bestimmten Höhe durch die Widerstandsänderung zwischen zwei durch den Tank gespannte Drähte. Eine weitere Methode besteht darin, $\phi$ über Temperatur- und Konzentrationsmessungen zu bestimmen. Dies gelingt jedoch nur, wenn innerhalb der porösen Schicht keine vertikale Advektion (keine Konvektion) stattfindet (z. B. Shirtcliffe \& Kerr, 1992). In den hier durchgeführten Experimenten trifft das jedoch nur bis zum Einsatz der Plumekonvektion zu. Da eine Störung der Strömungsstrukturen durch diese aufwendigen Messmethoden in dem rotierenden Tank nicht ausgeschlossen werden kann, wird in dieser Arbeit die im Folgenden beschriebene nachträgliche Bestimmung der Konzentration verwendet.

Zur Bestimmung des gesamten auskristallisierten Materials wird in dieser Arbeit die Änderung der Konzentration in der flüssigen Phase betrachtet. Dazu geht man von einer konstanten Konzentration in der gesamten flüssigen Phase aus, was bis auf einen kleinen Übergangsbereich zur Dendritenschicht gut erfüllt ist (Abb. 6.5). Diese Annahme wird durch Ergebnisse von Chen et al. (1994) bestätigt. Während des Experiments werden geringe Mengen an $\mathrm{NH}_{4} \mathrm{Cl}-\mathrm{H}_{2} \mathrm{O}$ (ca. $1 \mathrm{ml}$ ) mit einer $150 \mathrm{~mm}$ langen und $1 \mathrm{~mm}$ dicken Injektionsnadel aus dem Zylinder entnommen. Die Proben werden in dicht verschlossene Behälter gegeben und die Konzentrationen über die Änderung des Brechungsindexes bestimmt. Der Brechungsindex wird mit einem ABEE-Refraktometer gemessen, dessen Probenbühne temperierbar ist, so dass Temperatureinflüsse auf den Brechungsindex ausgeschlossen werden können.

Über eine Kalibrierung (Abb. 6.2) kann dem gemessenen Index $n_{D}$ eindeutig eine Konzentration zugeordnet werden. Der Brechungsindex wird für zehn angesetzte Konzentrationen im Bereich zwischen 0 und 25 gew\% bestimmt. Er ändert sich um einen Faktor von $2 \cdot 10^{-3}$ linear pro gew $\%$. Das verwendete Refraktometer hat eine relative Messgenauigkeit des Brechungsindexes von $2 \cdot 10^{-4}$ (Herstellerangabe). Es ist also möglich, die Konzentration auf $\pm 0,2$ gew\% genau zu bestimmen. Dies ist ausreichend, da die typische Konzentrationsänderung während eines Experiments 4-6 gew\% beträgt.

Der prozentuale Anteil an festem Material $\phi$ in der porösen Schicht ist definiert als 


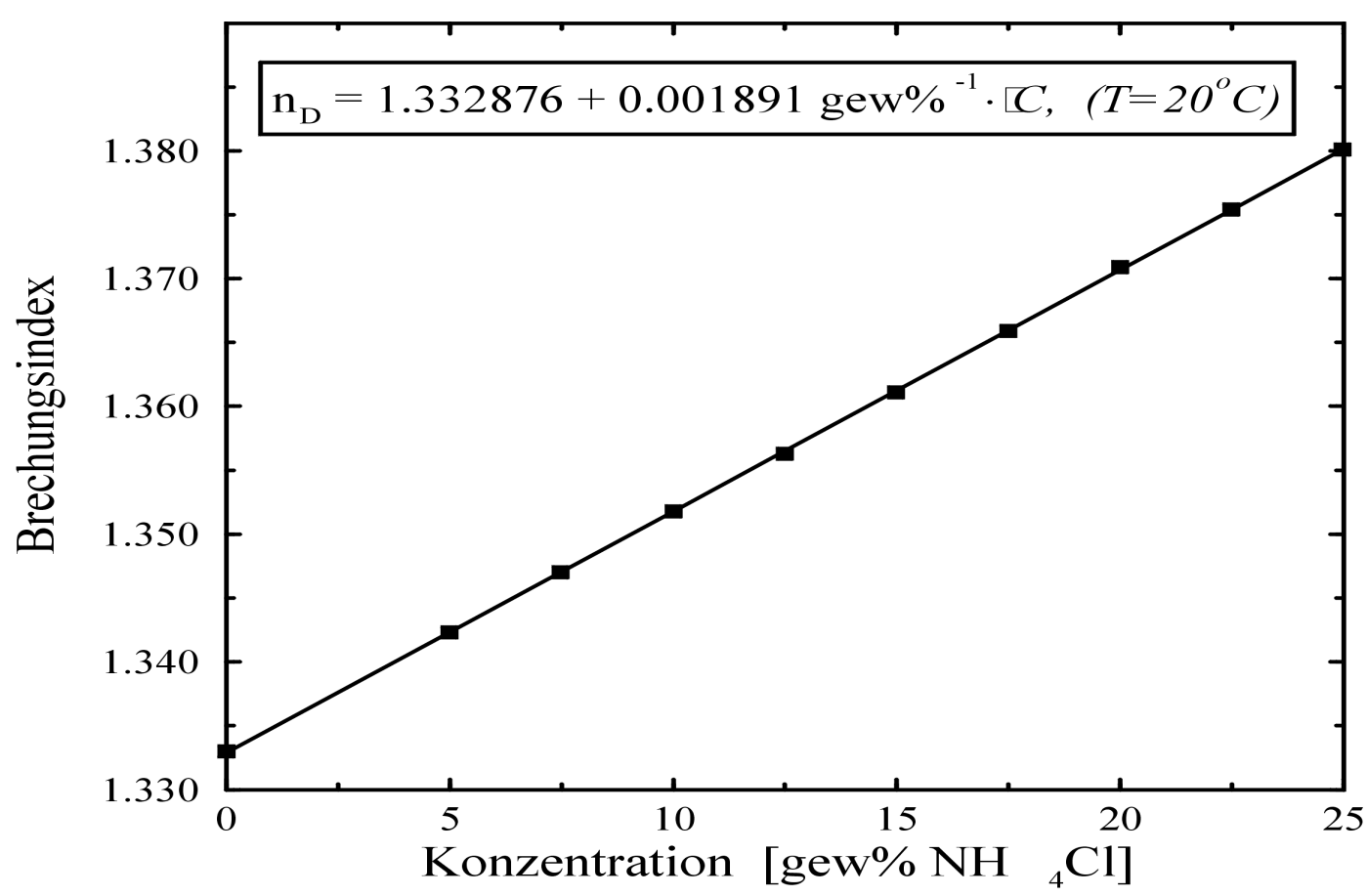

Abbildung 6.2: Kalibrierungskurve

Für zehn angesetzte Konzentrationen wird der Brechungsindex anhand von mindestens drei Proben für jede Konzentration mehrmals bestimmt. Die Abweichungen der Konzentrationen der einzelnen Proben sowie der einzelnen Konzentrationsmessungen sind so gering, dass die Fehlerbalken in der Größe der Quadrate liegen.

das Verhältnis von festem Volumen $V_{S}$ zum gesamten Volumen $V_{\text {ges }}$, das durch den Zylinderradius $r$ und die Höhe $H$ der Dendritenschicht definiert ist:

$$
\phi=\frac{V_{S}}{V_{\text {ges }}}, \quad V_{\text {ges }}=\pi r^{2} H
$$

Unter der Annahme einer homogenen Konzentration kann über die Messung der zeitlichen Änderung der Konzentration in der flüssigen Phase $C_{L}(t)$ und der Schichthöhe $H(t)$ auf Grund der Massenerhaltung direkt die Masse $M_{S}$ an auskristallisiertem Ammoniumchlorid und damit $\phi$ berechnet werden:

$$
\phi=\frac{M_{S}}{\rho_{S} \cdot V_{\text {ges }}}
$$

Die Dichte $\rho_{S}$ kann dabei als konstant angesehen werden. Diese Methode liefert jedoch keine Konzentrationsprofile durch die Schicht, sondern nur die zeitliche Entwicklung von $\phi$ gemittelt über die gesamte Schichthöhe. 


\section{Kapitel 7}

\section{Ergebnisse: Dendritisches Wachstum}

Im Folgenden werden die Ergebnisse des Kristallisationsprozesses dargestellt. Der erste Teil beschreibt die Beobachtungen, die allgemein während des Experiments sowohl mit, als auch ohne Rotation gemacht werden. In Abhängigkeit von der Unterkühlung entstehen z. B. auffällige Strukturen (Protokamine) in der Schicht, die in engem Zusammenhang mit den sich später ausbildenen Kaminen gebracht werden können. Im zweiten Teil wird untersucht, ob die Corioliskräfte einen Einfluss auf das Wachstum der Dendriten in der porösen Schicht sowie auf die Wachstumsrate dieser Schicht hat.

\subsection{Globale Beobachtungen des Wachstums}

Nach dem Einschalten der Kühlung bilden sich nach kurzer Zeit (bei $\Delta T>10 \mathrm{~K}$ und $\Theta<3 \mathrm{~K}$ in weniger als $1 \mathrm{~min}$ ) homogen verteilte Kristallkeime auf der Bodenplatte, die in Form von Dendriten weiter anwachsen. Liegt die Temperatur der Lösung $T_{2}$ um mehr als $3 \mathrm{~K}$ oberhalb der Liquidustemperatur, so kann sich die Keimbildung verzögern. So kam es in einem Versuch mit $\Delta T=12 \mathrm{~K}$ und $\Theta=11,3 \mathrm{~K}$ erst nach etwa 9 min zur Keimbildung. Die Keime entstehen bevorzugt an den Rändern des Gefäßes oder an Kanten bzw. Rillen auf der Platte, an diesen Stellen findet die durch die verringerte Grenzflächenenergie begünstigte heterogene Keimbildung statt (siehe z. B. Haasen, 1994, S. 54). Die Anzahl und Größe der Keime sowie ihre Wachstumsgeschwindigkeit hängen ebenfalls von $\Delta T$ und $\Theta$ ab und bestimmen, wie im folgenden beschrieben wird, wesentlich den weiteren Verlauf der Kristallisation. 


\subsubsection{Starke Unterkühlung}

Ist die Unterkühlung $\Delta T>15 \mathrm{~K}$ und $\Theta \leq 1 \mathrm{~K}$, dann bilden sich zahlreiche kleine Keime homogen über die gesamte Kühlplatte verteilt (Abb. 7.1 A). Die Platte ist nach typischerweise 5-7 min vollständig bedeckt und es ist eine nahezu gleichmäßige Oberfläche mit einer Rauhigkeit von weniger als $1 \mathrm{~mm}$ entstanden (Abb. 7.1 B). Die Schichtmächtigkeit beträgt zu diesem Zeitpunkt etwa 2-3 mm. Die Wachstumsfront fest/flüssig steigt dann nahezu planar weiter nach oben.

Die Wachstumsgeschwindigkeit ist für $\Delta T>15 \mathrm{~K}$ sehr groß und damit die Strömung entsprechend stark. Auf Grund der starken Strömung können aus der Wachstumsfront kleine Dendritenfragmente (Abb. 7.2) mit einem Durchmesser von 0,02 $\mathrm{mm}$ bis $0,1 \mathrm{~mm}$ herausgerissen werden, die in der flüssigen Phase mitschwimmen. Diese können zur Abschätzung der Strömungsgeschwindigkeit verwandt werden (s. Kap. 6.4). Sie steigen typischerweise bis in Höhen von 10 bis $20 \mathrm{~mm}$, einzelne Fragmente erreichen bis zu $40 \mathrm{~mm}$, bevor sie wieder nach unten sinken. Die ersten Fragmente werden abgerissen, wenn die Kristallschicht eine Mächtigkeit von etwa $3 \mathrm{~mm}$ hat. Oberhalb einer planaren Wachstumsfront sind die Fragmente gleichmässig verteilt. Ist die Kristallschicht jedoch noch nicht geschlossen, treten sie bevorzugt oberhalb der höchsten Erhebungen auf (Abb. 7.2). Da im Laufe der Zeit weniger Material erstarrt (vgl. Kap. 7.8), nimmt auch die Stärke der Strömung ab, so dass keine Dendriten mehr abgerissen werden.

\subsubsection{Geringe Unterkühlung}

Bei geringer Unterkühlung $\Delta T<15 \mathrm{~K}$ und $\Theta>2 \mathrm{~K}$ entstehen weniger, jedoch größere Keime, wie man auch nach der klassischen Keimbildungstheorie erwartet (Haasen, 1994, S. $52 \mathrm{ff}$ ). Sie wachsen langsamer als bei starker Unterkühlung, da die treibende Kraft für die Kristallisation geringer ist. $\mathrm{NH}_{4} \mathrm{Cl}$ ist ein kubisches System der Vorzugswachstumsrichtung $<100>$, somit entstehen kubische Kristallstrukturen aus einer Vielzahl Dendriten, deren äußeres Erscheinungsbild (Habitus) von den $\{111\}$ Ebenen gebildet wird. Der Habitus stellt somit eine Pyramide mit einer quadratischen Grundfläche dar (Abb. 7.3).

In der Mitte dieser Pyramide kann man tiefergehende Hohlräume erkennen (Abb. 7.3), ähnlich den später zu beobachtenden Kaminen. Im folgenden Abschnitt werden diese „Protokamine“ genauer untersucht. 

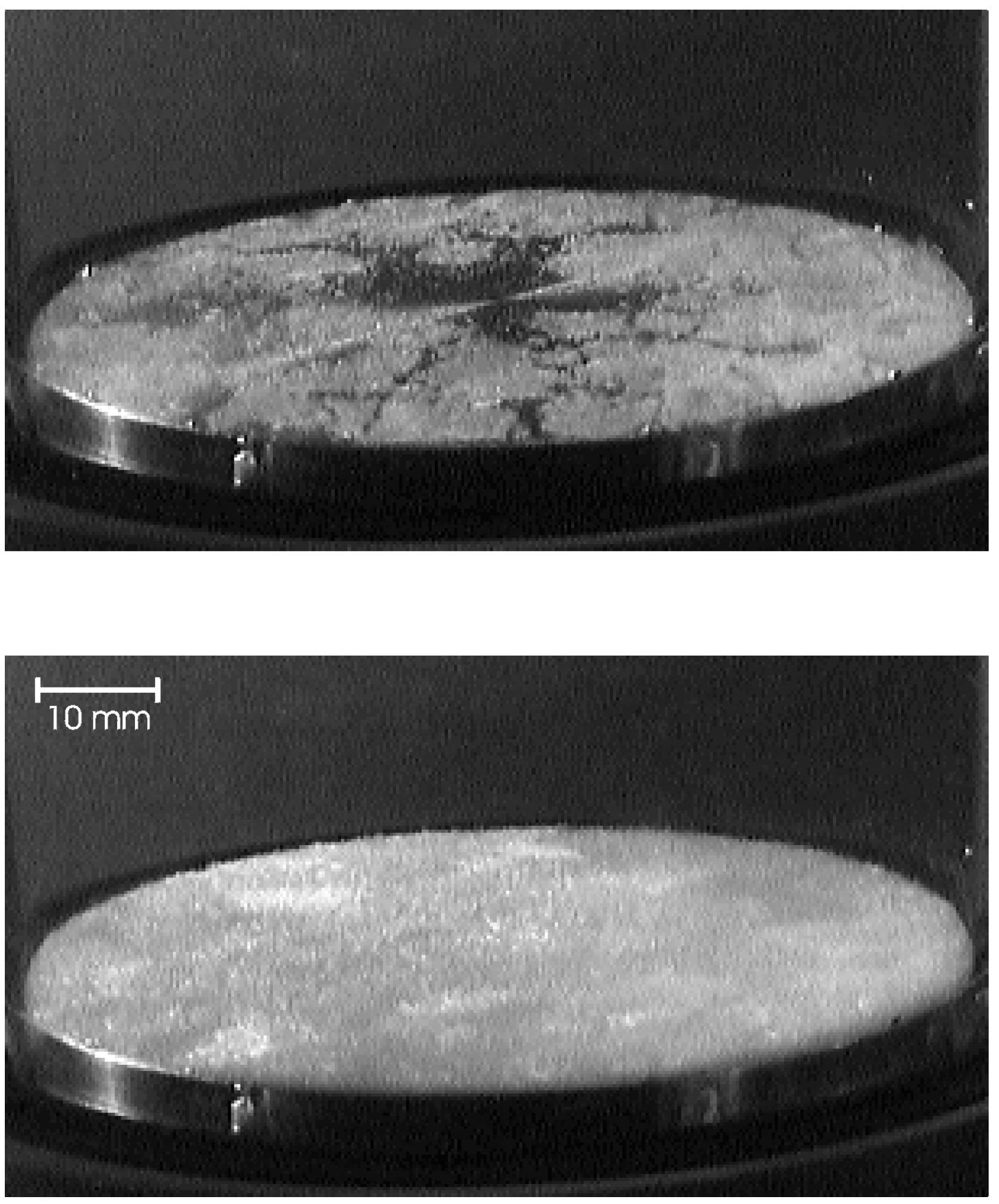

Abbildung 7.1: Keime

A: Die Kühlplatte ist bereits 30 s, nachdem die Kühlung begonnen hat, mit einer Vielzahl kleiner, kristalliner Dendriten bedeckt.

B: Nach 9 min ist die Platte gleichmäßig bedeckt und die Schicht hat eine Höhe von etwa $4 \mathrm{~mm}$ erreicht. $\left(\Delta T=16,5 \mathrm{~K}, C_{\circ}=26 \mathrm{gew} \%, \theta=3,4 \mathrm{~K}, \Omega=0 \frac{\mathrm{rad}}{\mathrm{s}}\right)$ 


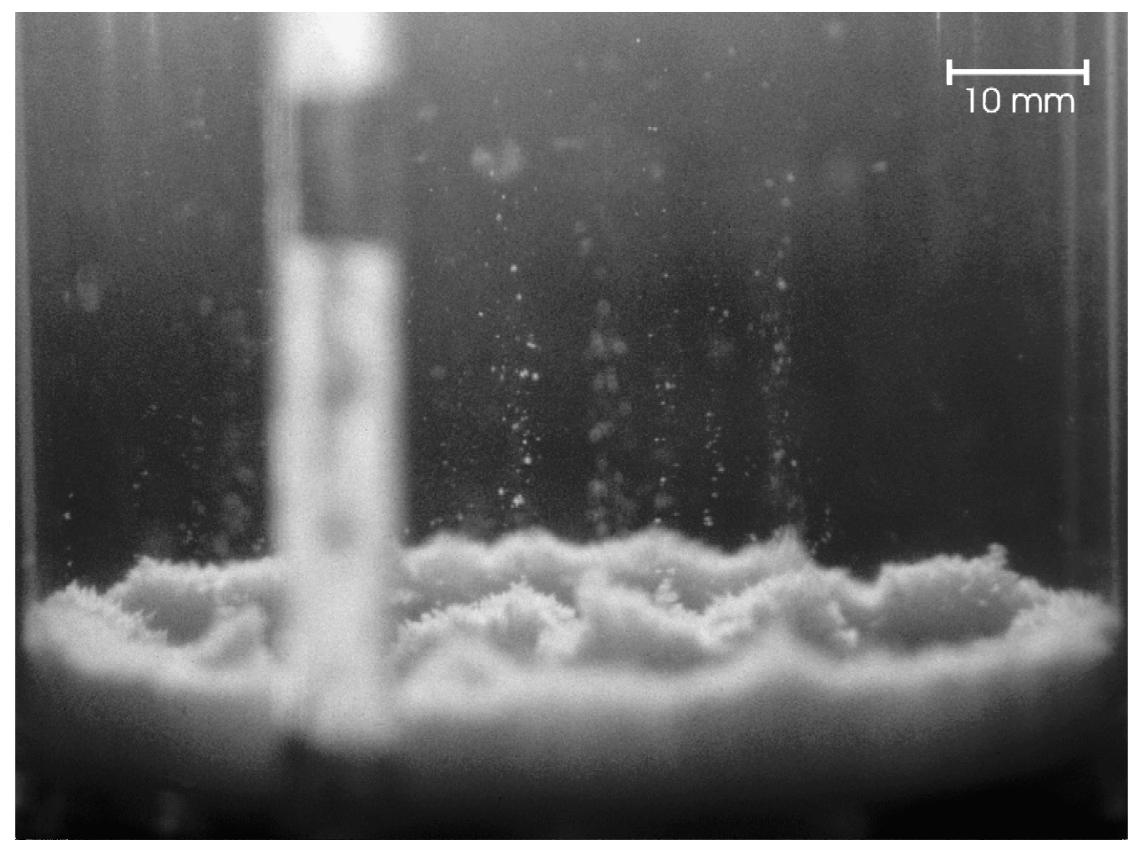

Abbildung 7.2: Kristallfragmente

Die Aufströme führen kleine Dendritenfragmente mit sich, die ab einer Höhe von 20 bis $30 \mathrm{~mm}$ wieder nach unten sinken. Sie werden an der Wachstumsfront oder an den Kaminwänden abgerissen. $\left(\Delta T=15,5 \mathrm{~K}, C_{\circ}=26 \mathrm{gew} \%, \theta=0,9 \mathrm{~K}, \Omega=0 \frac{\mathrm{rad}}{\mathrm{s}}\right)$

\subsection{Protokamine}

In der Anfangsphase des Kristallwachstums können oft Defekte bzw. Bereiche mit erhöhter Porosität in den Kristallen beobachtet werden. Um dieses Phänomen mit möglichst geringen Randeffekten genauer untersuchen zu können, wird eine Reihe von Experimenten in einem größeren, quadratischen Plexiglas-Tank mit einer Grundfläche von $200 \mathrm{~mm} \times 200 \mathrm{~mm}$ durchgeführt. Die Anfangskonzentration $C_{\circ}$ in diesen Versuchen beträgt 26 gew $\%$ mit drei verschiedenen Unterkühlungen $\Delta \mathrm{T}=5 \mathrm{~K}, 7 \mathrm{~K}$ und 9 K. Die Kristallisation wird von oben mit Hilfe einer CCD-Kamera beobachtet. Die zeitliche Entwicklung des Kristallisationsprozesses wird in Abbildung 7.4 für $\Delta T=5 \mathrm{~K}$ exemplarisch erläutert.

Sobald die Lösung auf einige Grad unterhalb ihrer Liquidustemperatur abgekühlt ist, entstehen auf der Kühlplatte unregelmäßig verteilt Kristallkeime. Sie wachsen zu den in Kapitel 7.1.2 beschriebenen quadratischen Pyramiden an und bereits nach wenigen Minuten (Bild A, t=7 min) sind deutlich „Protokamine“ in der Mitte der größeren Kristallverbände entstanden. In den Diagonalen des Kristalls ist die Dichte der Dendriten ebenfalls geringer. Die Singularitäten der Dendriten entstehen dabei in den $<100>$ 

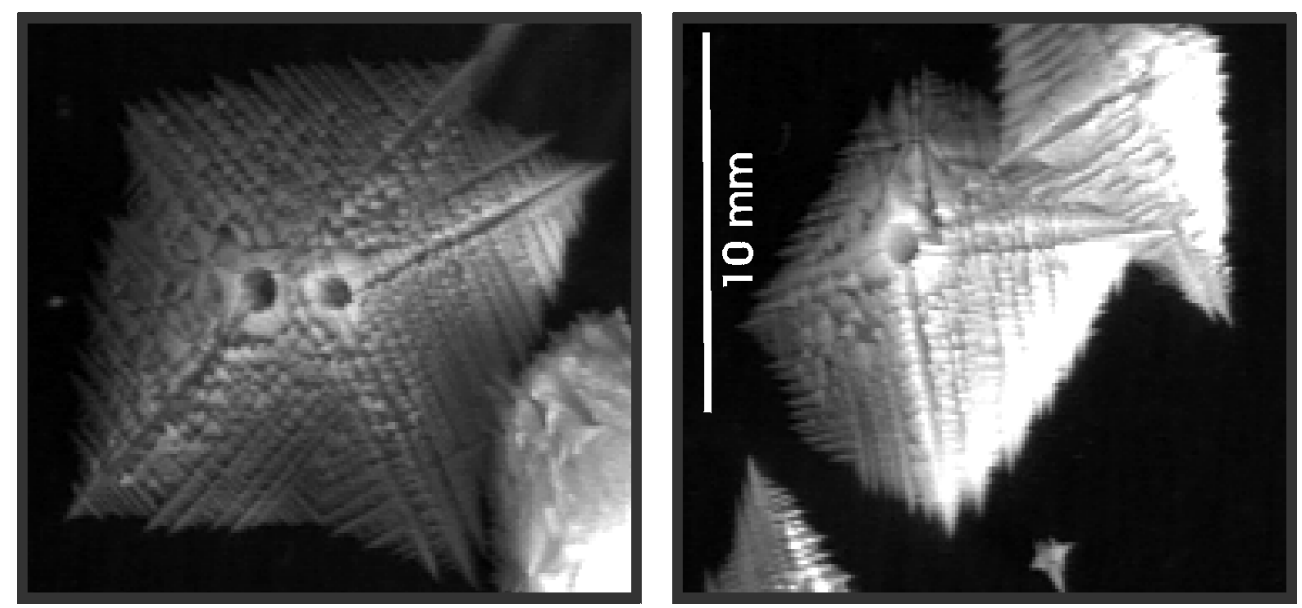

\section{Abbildung 7.3: Kristalle von oben mit Protokaminen}

In der Mitte der Kristallpyramide und in den Diagonalen bildet sich während des Wachstums ein hochporöser Bereich aus, der die Bildung von kaminähnlichen Strukturen (Protokamine) induzieren kann. $\left(\Delta T=5 \mathrm{~K}, C_{\circ}=26 \mathrm{gew} \%, \theta \approx 5 \mathrm{~K}, \Omega=0 \frac{\mathrm{rad}}{\mathrm{s}}\right.$ )

Richtungen. Mit der Zeit wachsen die einzelnen Pyramiden weiter zusammen (Abb. 7.4 B-D) und bilden nach etwa 50 min eine geschlossene Kristallschicht. Diese Schicht ist mit einem Muster von „Wegen" und Kaminen durchsetzt (Abb. 7.4 E, F), wobei die Wege häufig in den Kaminen münden. Im Laufe des Kristallisationsprozesses füllen sich die hochporösen bzw. kristallfreien Regionen weiter mit Dendriten, und ein unregelmäßiges Muster von Kaminen bleibt zurück. .

Im Folgenden wird der Zusammenhang zwischen den Protokaminen und den vollentwickelten Kaminen untersucht. Hierzu sind in den Einzelbildern der Abbildung 7.4 die Entstehungsorte der Keime (und damit der Protokamine), die hochporösen Diagonalen der Kristalle und die Orte der späteren Kamine einzeln markiert. Anschließend werden die Markierungen zu den verschiedenen Zeitpunkten übereinander projiziert (Abb. 7.5). Der Vergleich ergibt, dass die Markierungen jeweils nur leicht gegeneinander verschoben sind. In den meisten Fällen fällt die Position eines Kamins mit dem Ort eines Protokamins zusammen oder seltener in den Bereich einer Kristalldiagonalen. Die Wege innerhalb der Schicht können gut mit den Diagonalen korrelliert werden.

Man beachte, dass aus Gründen der Übersichtlichkeit nur die ganz zu Anfang (Abb. 7.4 A) entstandenen Protokamine eingezeichnet sind. Nimmt man die wenigen später gebildeten hinzu, so stellt man fest, dass fast alle Kamine über Protokaminen oder Diagonalen entstanden sind.

In Abbildung 7.5 erkennt man, dass die Position eines Protokamins/Kamins nicht 

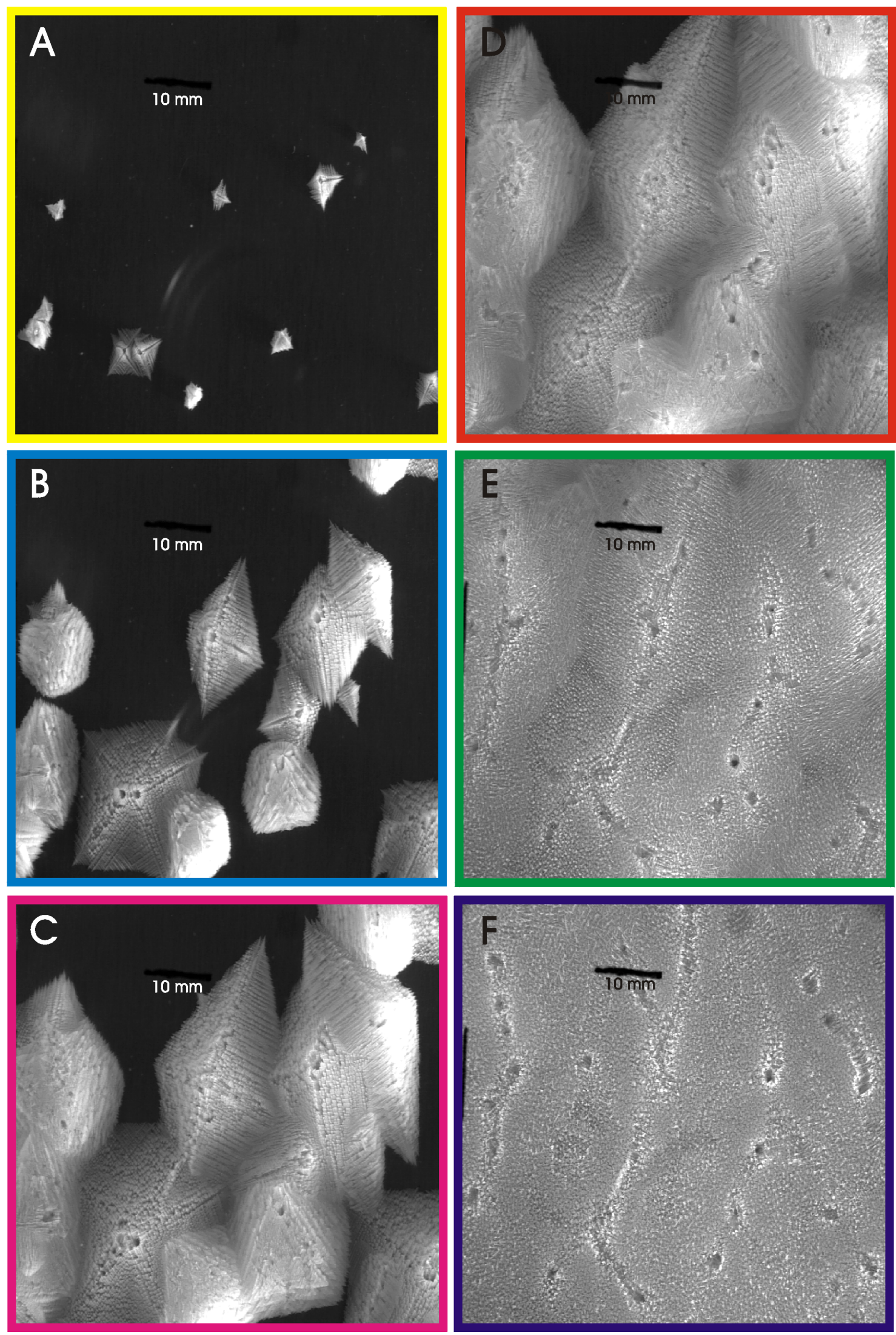

Abbildung 7.4: Dendritenwachstum

Zeitliche Entwicklung der Kristallschicht. $\left(\Delta T=5 \mathrm{~K}, C_{\circ}=26 \mathrm{gew} \%, \theta \approx 5 \mathrm{~K}, \Omega=0 \frac{\mathrm{rad}}{\mathrm{s}}\right)$ $A: t=7 \min , B: t=17 \min , C: t=27 \min , D: t=37$ min, $E: t=67$ min, $F: t=97$ min 

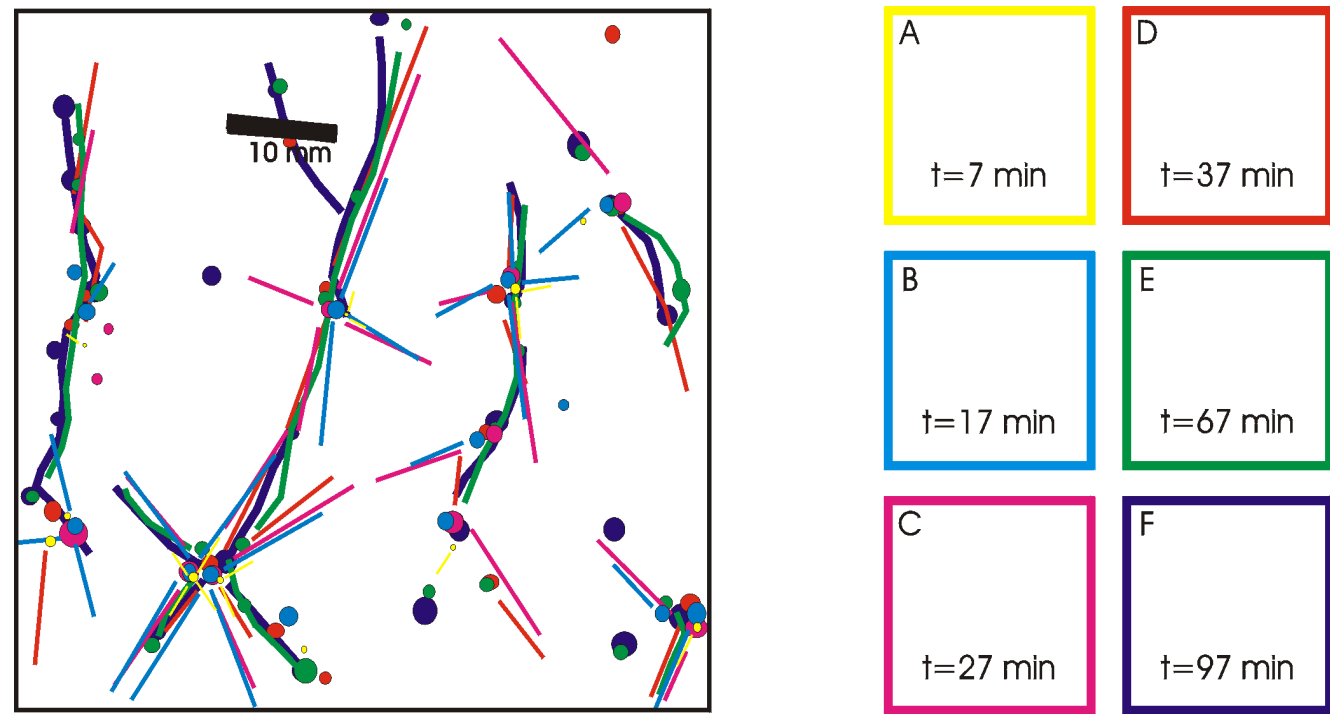

Abbildung 7.5: Markierung der Pfade und (Proto-) Kamine in der Kristallschicht (aus Abb. 7.4) zu verschiedenen Zeitpunkten

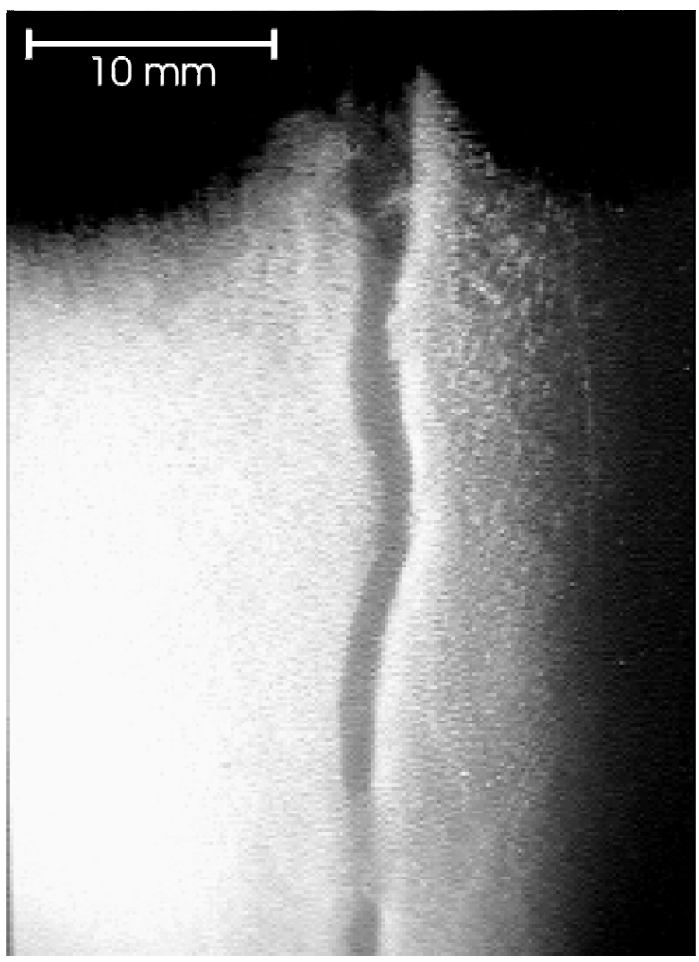

Abbildung 7.6: Kaminquerschnitt

Schnitt durch einen tief in die Dendritenschicht reichenden Kamin Der Durchmesser beträgt etwa 2,5 mm. Der vertikale Verlauf ist gewunden. An der Schichtoberfläche sind einzelne Dendrite mit einem mittleren Abstand von 0,41 $\mathrm{mm} z u$ erkennen. $\left(\Delta T=24,5 \mathrm{~K}, C_{0}=26\right.$ gew $\%$, $\left.\theta=0,5 K, \Omega=2,1 \frac{\mathrm{rad}}{\mathrm{s}}, E=3,8 \cdot 10^{-5}\right)$. 


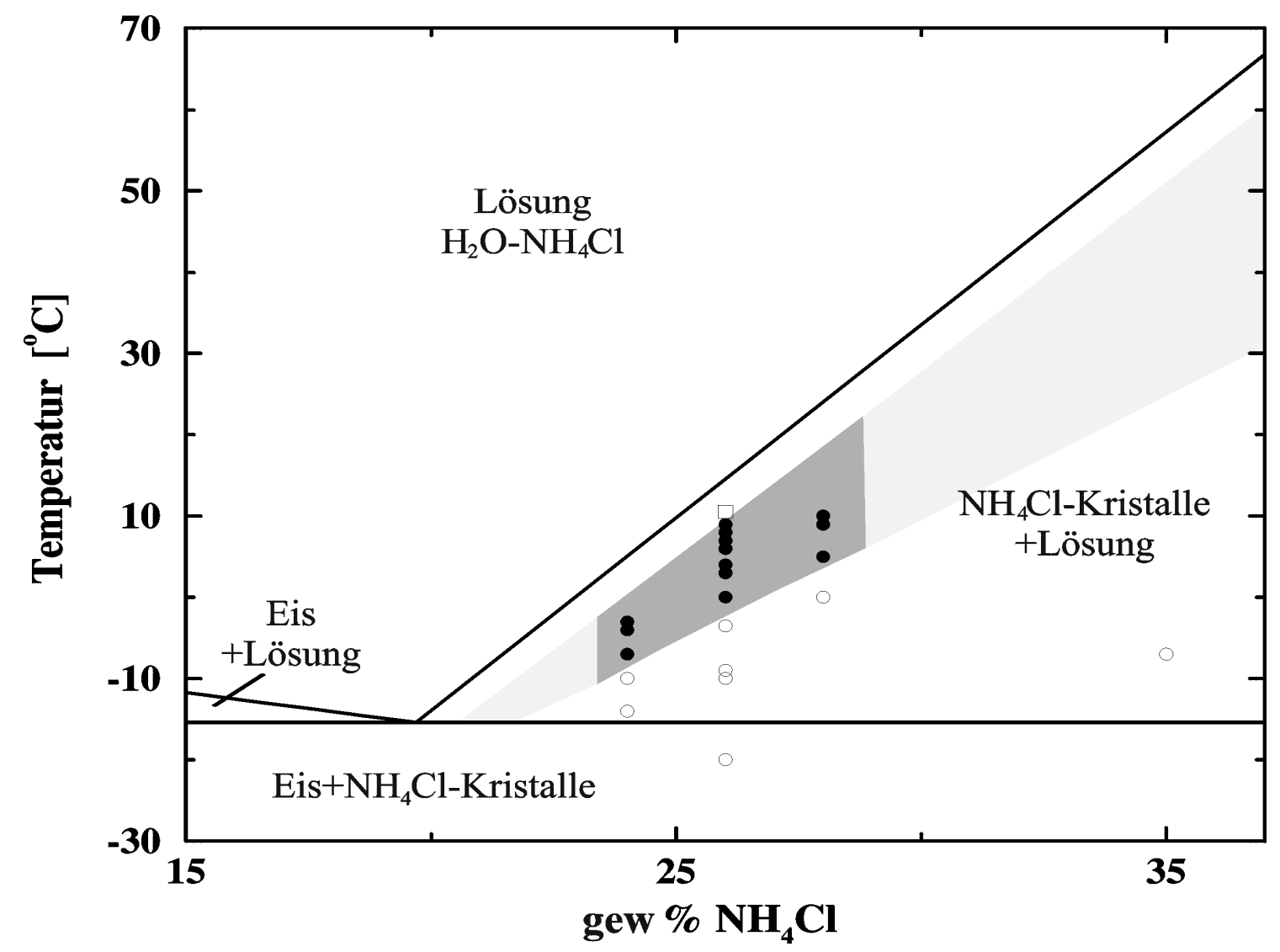

Abbildung 7.7: Bereich, in dem Protokamine entstehen

In das Phasendiagramm der wässrigen Ammoniumchloridlösung sind alle durchgeführten Experimente mit $C_{o}$ und $T_{1}$ eingetragen. Mit gefüllten Kreisen sind diejenigen Versuche gekennzeichnet, bei denen Protokamine entstanden sind. In Versuchen, die mit offenen Kreisen markiert sind, konnten keine Protokamine beobachtet werden (offenes Quadrat: keine Plumekonvektion). Die grau gekennzeichnete Region gibt den Bereich an, in dem es zum Entstehen von Protokaminen kommen könnte.

genau fixiert ist, sondern im Laufe der Zeit etwas variiert. Dieses wird deutlich, wenn man den Querschnitt eines Kamins (Abb. 7.6) betrachtet. Der Kamingang ist nicht vollkommen gerade, sondern windet sich nach oben. Die horizontalen Variationen betragen etwa 1-2 mm, dies entspricht den Schwankungen in den Markierungen.

Durch dieses Experiment wird deutlich, dass bereits zu Anfang der Kristallisation die Positionen der Kamine bei der späteren Konvektion festgelegt werden.

In Abbildung 7.7 sind die durchgeführten Experimente mit ihrer jeweiligen Kühltemperatur $T_{1}$ und Anfangskonzentration $C_{\circ}$ in das $\mathrm{NH}_{4} \mathrm{Cl}-\mathrm{H}_{2} \mathrm{O}$ Phasendiagramm eingetragen worden. Die unterschiedlichen Symbole kennzeichnen: Entstehung von Proto- 
kaminen (gefüllte Kreise), keine Beobachtung von Protokaminen (offene Kreise) und keine Plumekonvektion (offenes Quadrat). Der hellgrau schattierte Bereich markiert wo die Entstehung von Protokaminen und damit eine frühe Form der Plumekonvektion (vgl. Kap. 8) zu erwarten ist. In dem hier untersuchten Parameterbereich (dunkelgrau) werden sie ab einer Unterkühlung von $\Delta T<12 \mathrm{~K}$ (bei $C_{\circ}=24$ gew\%) beobachtet; der Existenzbereich vergrössert sich auf $\Delta T<20 \mathrm{~K}$ bei $C_{\circ}=28$ gew\%. Das Auftreten von Protokaminen ist unabhängig von der Rotation, im gesamten untersuchten Bereich von $\Omega=0-10,5 \frac{\mathrm{rad}}{\mathrm{s}}$ werden Protokamine beobachtet.

\subsection{Einfluss der Rotation auf die Kristallschicht}

Im Einzelnen wird nun der Einfluss der Rotation auf das Wachstum der Dendritenschicht und der Kamine untersucht. Betrachtet werden die Wachstumsrichtung der Dendrite, die Porosität bzw. Permeabilität der Schicht und die Wachstumsgeschwindigkeit.

\subsubsection{Wachstumskinetik}

In diesem Abschnitt soll das Wachstum der porösen Dendritenschicht genauer untersucht werden. Abbildung 7.8 zeigt das Wachstum der Dendritenschicht für vier unterschiedliche Winkelgeschwindigkeiten $\left(\Omega=0,20,40,80 \frac{\mathrm{rad}}{\mathrm{s}}\right)$, zusätzlich ist die Plumeanzahl während des Experiments eingetragen, auf die im Kapitel 8 eingegangen wird.

Das Wachstum der Dendritenschichthöhe lässt sich in drei unterschiedliche Bereiche einteilen, die zunächst ohne Rotation betrachtet werden sollen.

Im Frühstadium des Wachstums, direkt nach Beginn der Kristallisation, bestimmt die Unterkühlung $\Delta T$ das Wachstum. Nach dem Standardmodell für die Wachstumsgeschwindigkeit $v_{\circ}$ von Dendriten $v_{\circ} \propto \Delta T^{2}$ (Haasen, 1994, S. 56) wird das Wachstum durch die Diffusion unmittelbar vor der Dendritenspitze bestimmt. An die Wachstumskuven wird daher eine Tangente in $t=0$ angelegt und somit $v_{\circ}$ bestimmt. In Abbildung 7.9 sind die gemessenen Wachstumsgeschwindigkeiten über die Unterkühlung aufgetragen, im Rahmen der Messgenauigkeit trifft dieses einfache Modell zu.

Im weiteren Verlauf wird das Wachstum der Dendritenschicht durch die Wärmediffusion, durch die schon vorhandene Schicht, bestimmt. Theoretische Analysen von Huppert \& Worster (1985) und Worster (1986) zeigen, dass die Schichtdicke $H$ eine Funktion 


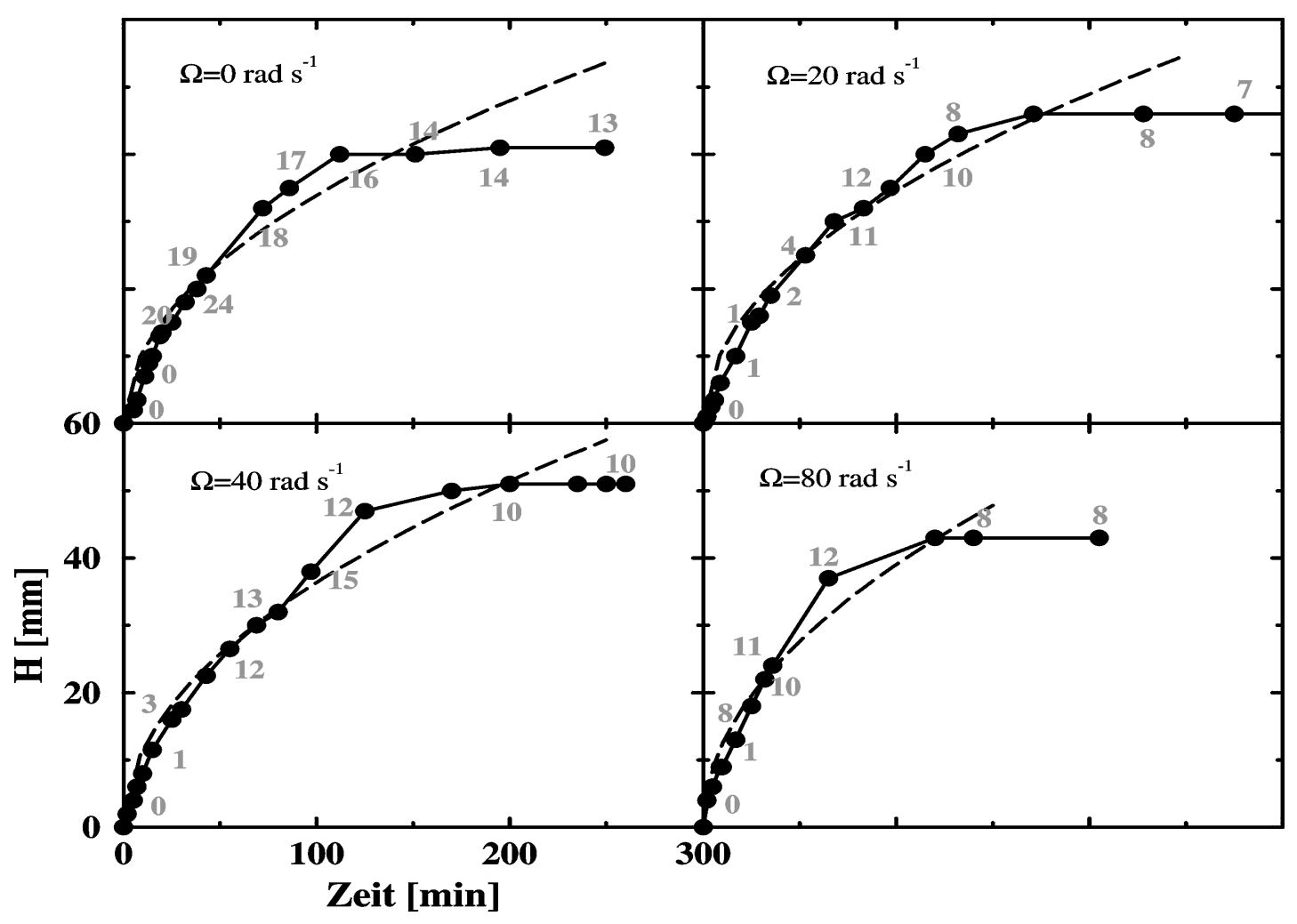

Abbildung 7.8: Schichtwachstum bei unterschiedlichen Winkelgeschwindigkeiten

Punkte repräsentieren die Schichthöhe aus den Experimenten und Zahlen geben die Anzahl der Plumes zum entsprechenden Zeitpunkt wieder. An die Messwerte wird eine Modellkurve (gestrichelte Linie) nach Gleichung 7.1 angepaßt. $\left(\Delta T=24 \mathrm{~K}, \mathrm{C}_{\mathrm{0}}=28 \mathrm{gew} \%\right.$, $\theta<2 K)$

von $\sqrt{t}$ ist, falls keine Konvektion innerhalb der Dendritenschicht stattfindet und das Wachstum somit diffusionskontrolliert ist:

$$
H=2 \cdot \lambda \cdot \sqrt{\kappa_{c} \cdot t}
$$

$\lambda$ ist der hier interesssierende Wachstumskoeffizient. Er ist von der Liquidustemperatur, der latenten Wärme, den thermischen und chemischen Diffusionskoeffizienten und der Unterkühlung abhängig. $\kappa_{c}$ bezeichnet die chemische Diffusivität. Tait \& Jaupart (1989) und Chen et al. (1993) zeigen, dass diese Gleichung auch für Schichten mit Konvektion Gültigkeit besitzt. Bis etwa 100 bis 150 min kann das Schichtwachstum akzeptabel durch die Gleichung 7.1$]$ beschreiben werden.

Für größere Zeiten dagegen wächst die Schichtdicke nicht weiter an. In diesem Spätsta- 


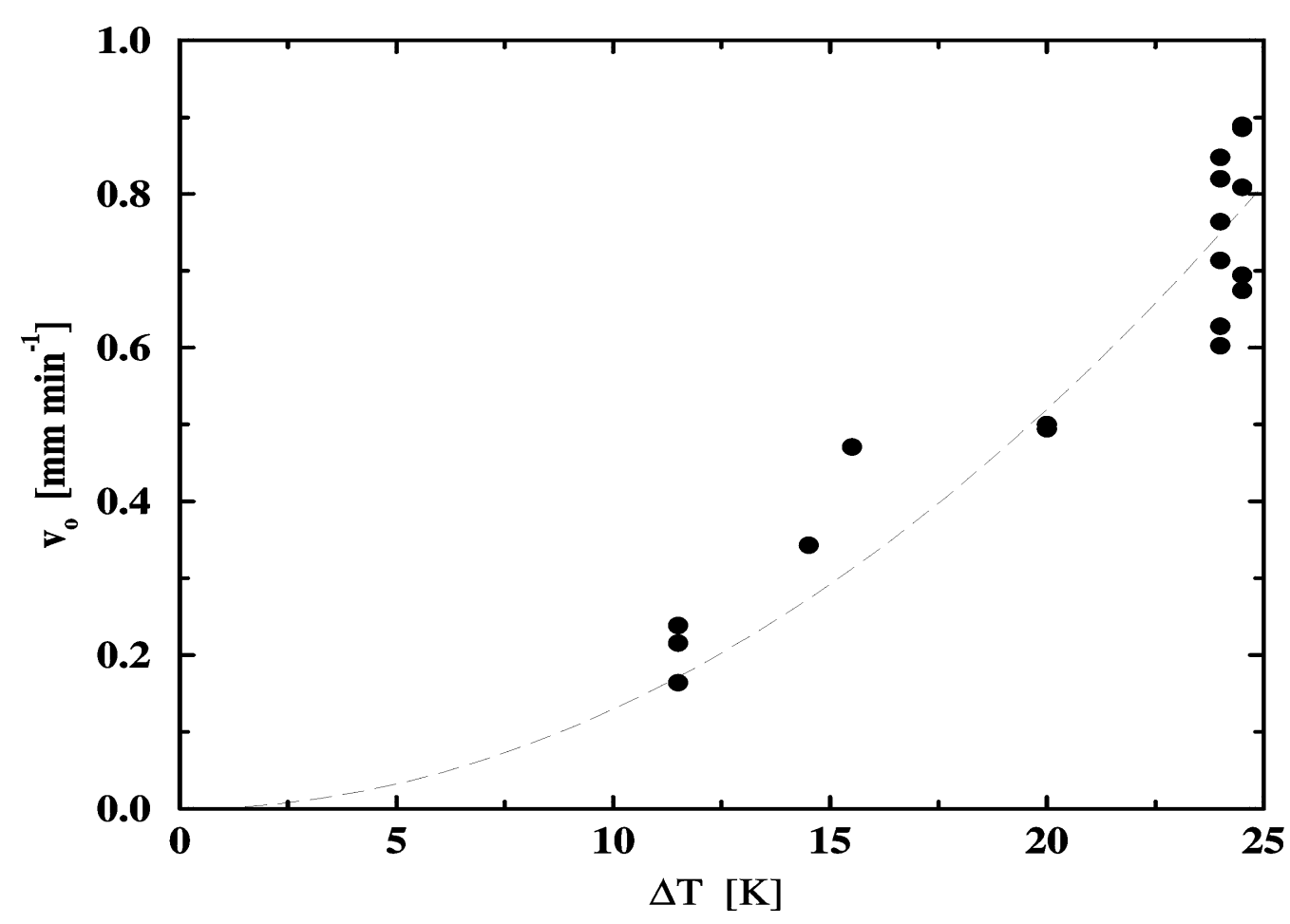

\section{Abbildung 7.9: Wachtumsgeschwindigkeit}

Die Wachstumsgeschwindigkeit kann durch einem quadratisch Anstieg mit der Unterkühlung beschrieben werden. $(\theta<2 K)$

dium wird das Wachstum dadurch begrenzt, dass die Konzentration von $\mathrm{NH}_{4} \mathrm{Cl}$ im restlichen Volumen weit reduziert ist und somit fast kein Material zum Kristallisieren übrig bleibt.

Um den Einfluss der Rotation auf das Wachstum zu untersuchen, wird $\lambda$ für verschiedene Winkelgeschwindigkeiten bestimmt. Abbildung 7.10 zeigt $\lambda$ in Abhängigkeit von der Winkelgeschwindigkeit $\Omega$ für zwei unterschiedliche Unterkühlungen. Für $\Delta T=24 \mathrm{~K}$ variiert $\lambda$ zwischen 4,0 und 6,0. Die Streuung der Messwerte ist jedoch irregulär und wahrscheinlich auf nicht kontrollierte Unterschiede in den Experimenten sowie Messungenauigkeiten zurückzuführen. Für $\Delta T=12 \mathrm{~K}$ variiert $\lambda$ weniger. Der Mittelwert ist mit etwa 1,8 niedriger, dies ist auf die geringere Wachstumsrate bei niedrigerer Unterkühlung zurückzuführen. Auch hier kann kein Einfluss der Rotation nachgewiesen werden. Diese Beobachtungen sind in Übereinstimmung mit den vorher vorgestellten Konzepten, dass die rotationsunabhängige Diffusion und nicht die Konvektion das Wachstum der Dendritenschicht limitiert. 


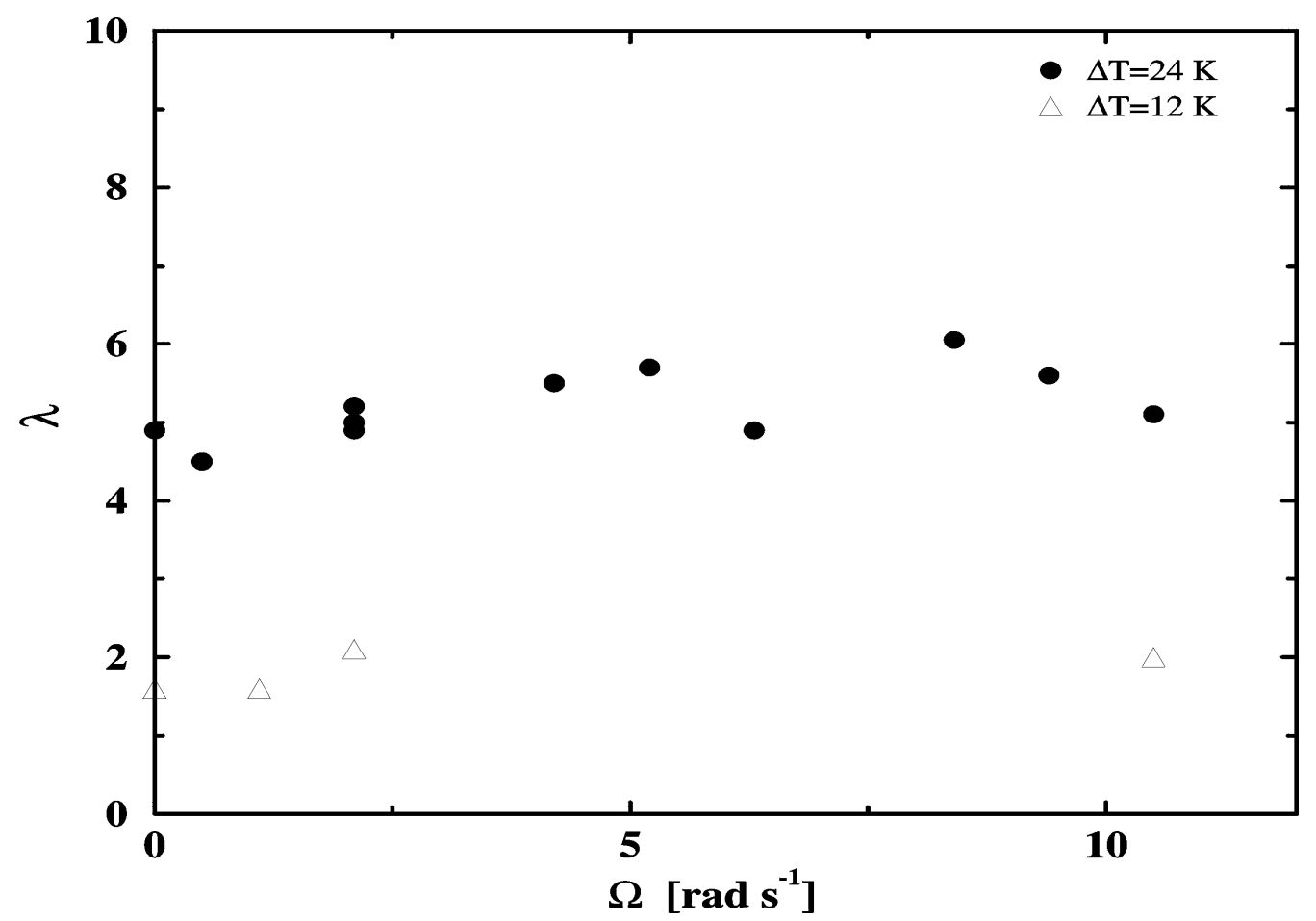

\section{Abbildung 7.10: Wachtumskoeffizient in Abhängigkeit von der Winkelge- schwindigkeit}

Der Wachstumskoeffizient $\lambda$ (für eine Unterkühlung $\Delta T$ ) zeigt keine signifikante Abhängigkeit von der Winkelgeschwindigkeit $\Omega$.

\subsubsection{Morphologie}

Der Einfluss der Rotation auf die inneren Schichteigenschaften wird exemplarisch an drei Experimenten mit den Winkelgeschwindigkeiten 0, 2,1 und 6,3 $\frac{\mathrm{rad}}{\mathrm{s}}\left(\mathrm{E}=3,8 \cdot 10^{-5}\right.$ bzw. 1,3 $10^{-5}$ ) untersucht. Abbildung 7.11 zeigt einen vertikalen Schnitt durch eine Kristallschicht aus dem mittlerer Bereich zwischen Rotationsachse und Gefäßwandung (bei $\Omega=6,3 \frac{\mathrm{rad}}{\mathrm{s}}$ ). Die Dendriten sind parallel zu g und damit zur Richtung des Wärmeflusses ausgerichtet. In der hier durchgeführten Untersuchung wird kein Einfluss durch die Rotation beobachtet. Da die Rotationsachse in Richtung der Gravitation zeigt, ist dieses auch nicht zu erwarten. In Tabelle 7.1 werden die Ergebnisse des Vergleichs zusammengefasst. Der Dendritendurchmesser $d$ stimmt in allen Experimenten nahezu überein. Die gemittelten Werte für den Dendritenabstand liegen in den unterschiedlichen Proben weiter auseinander. Der Messfehler liegt jedoch mit 0,1 mm in der gleichen Größenordnung wie die Streuung der Messwerte. 


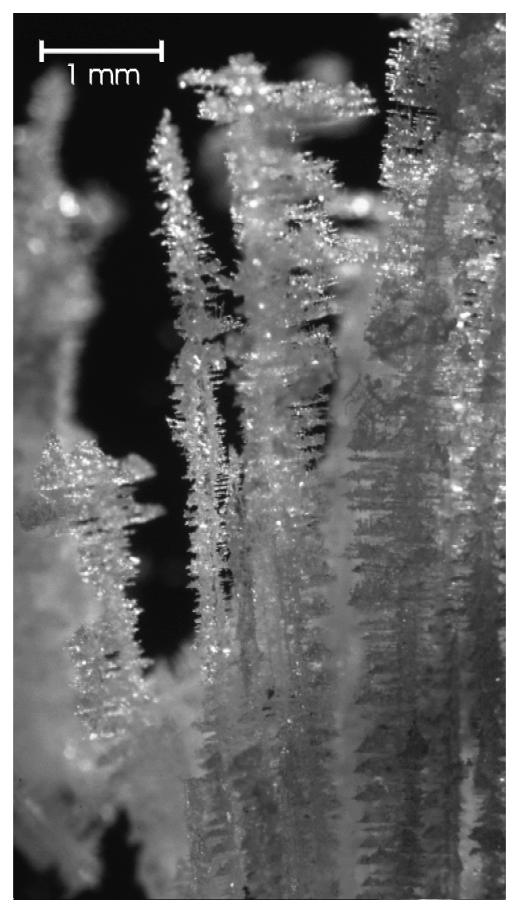
Abbildung 7.11: Dendrite bei hoher Rotati- onsrate
Vertikaler Schnitt aus dem mittleren Bereich der Kristallschicht zwischen Rotationsachse und Gefäßrand. Dendrite stehen parallel nebeneinan- der mit einem mittleren Abstand von 0,35 $\mathrm{mm}$. $\left(\Delta T=24,5 \mathrm{~K}, C_{\circ}=26 \mathrm{gew} \%, \theta=0,5 \mathrm{~K}, \Omega=6,3 \frac{\mathrm{rad}}{\mathrm{s}}\right.$, $\left.E=1,3 \cdot 10^{-5}\right)$

\begin{tabular}{|c|c|c|c|c|c|}
\hline$\Delta \mathrm{T}[\mathrm{K}]$ & $\mathrm{C}_{\circ}[$ gew $\%]$ & $\theta[\mathrm{K}]$ & $\Omega\left[\frac{\mathrm{rad}}{\mathrm{s}}\right]$ & $\mathrm{d}[\mathrm{mm}]$ & $\mathrm{PDA}[\mathrm{mm}]$ \\
\hline \hline 24,5 & 26 & 1,4 & 0 & 0,18 & 0,37 \\
\hline 24,5 & 26 & 0,5 & 2,1 & 0,17 & 0,41 \\
\hline 24,5 & 26 & 1,8 & 6,3 & 0,19 & 0,35 \\
\hline
\end{tabular}

Tabelle 7.1: Dendritenabstand

Gemittelter primärer Dendritenabstand PDA, gemessen nach Versuchsende

\subsubsection{Abschätzung der Permeabilität und Porosität}

Um die Permeabilität $\Pi$ abschätzen zu können, wird die Kristallschicht als ideales poröses Medium mit folgenden idealisierten Eigenschaften angenommen: Die Dendriten werden als Zylinder (oder sehr schlanke Kegel) angenähert, die parallel nebeneinander mit konstantem Abstand $P D A$ angeordnet sind. Sie werden von Flüssigkeit umströmt. Eine Einheitszelle der Schicht besteht aus einem festen Zylinder mit Radius $a=\frac{1}{2} \cdot d$, der sich zentral in einem flüssigen Zylinder mit Radius $b=\frac{1}{2} \cdot P D A$ befindet. Der feste Anteil $\phi$ der Zelle ist

$$
\phi=\frac{a^{2}}{b^{2}}
$$


Die Porosität $\chi$ kann im vorliegenden Fall als der flüssige Anteil der Zelle angenommen werden (Tait \& Jaupart, 1992). Sie ergibt sich damit zu:

$$
\chi=1-\phi=1-\frac{a^{2}}{b^{2}}
$$

Für die Permeabilität nach der Kozeny-Carman Gleichung (Bear, 1972), in der die Dendriten als sehr schlanke Kegel mit einem Basisdurchmesser $d \approx 2 \cdot a$ angenommen werden, ergibt sich:

$$
\Pi=\frac{\chi^{3} d^{2}}{180(1-\chi)^{2}}
$$

Experimentell kann die Porosität bzw. $\phi$ mit unterschiedlichen Methoden ermittelt werden:

In Kapitel 6.5 wird die Methode beschrieben, wie man die zeitliche Entwicklung von $\phi$ während des Experiments mit Hilfe von Konzentrationsmessungen in der flüssigen Phase bestimmen kann. Diese Methode kann jedoch erst angewandt werden, wenn die gesamte Flüssigkeit mit dem aufsteigenden Material geringerer Konzentration vermischt wird. Die Fingerkonvektion erreicht nur geringe Höhen, so dass die oberen Schichten nicht durchmischt werden. Im Frühstadium der Plumekonvektion sowie im Stadium der Protoplumes (siehe Kap. 8.1) ist die Durchmischung der aufsteigenden Flüssigkeit mit der Umgebung ebenfalls gering. In Abbildung 7.12 erkennt man, dass sich die Konzentration in der flüssigen Phase erst mit vollentwickelter Plumekonvektion deutlich ändert und kontinuierlich sinkt. Ab diesem Zeitpunkt kann die oben beschriebene Methode zur Abschätzung von $\phi$ eingesetzt werden. Abbildung 7.13 zeigt die kontinuierliche Abnahme von $\Pi$ während des Experiments in Abbildung 7.12 .

Eine weitere Möglichkeit, während des Versuchs $\phi$ zu erhalten, ist über den Abstand zweier benachbarter primärer Dendriten $(P D A)$ oder durch die Anzahl der Dendriten pro Fläche. Dabei wird wieder vorausgesetzt, dass die Dendriten einen Verband von parallelen Zylindern der Dicke $d$ mit gleichem Abstand voneinander bilden. Diese Annahme ist gut mit den Resultaten aus Kapitel 7.3.2 zu vereinbaren. In Tabelle 7.2 sind die Ergebnisse für $\phi, \chi$ und $\Pi$ eingetragen. Sie wird mit den Werten aus Tabelle 7.1 ermittelt. Die Ungenauigkeiten in der Bestimmung der Dendritenabstände und ihrer Durchmesser verursachen einen großen Fehler in $\Pi$.

Während des Experiments werden Aufnahmen von der Dendritenschicht (von oben und von der Seite) gemacht. Anhand dieser Bilder werden $d$ und PMA zum Zeitpunkt des Plumeeinsatzes ermittelt. Da die Werte große Messungenauigkeiten aufweisen, werden sie über mehrere Experimente gemittelt. Man erhält $\chi=0,3$ für den Einsatz 


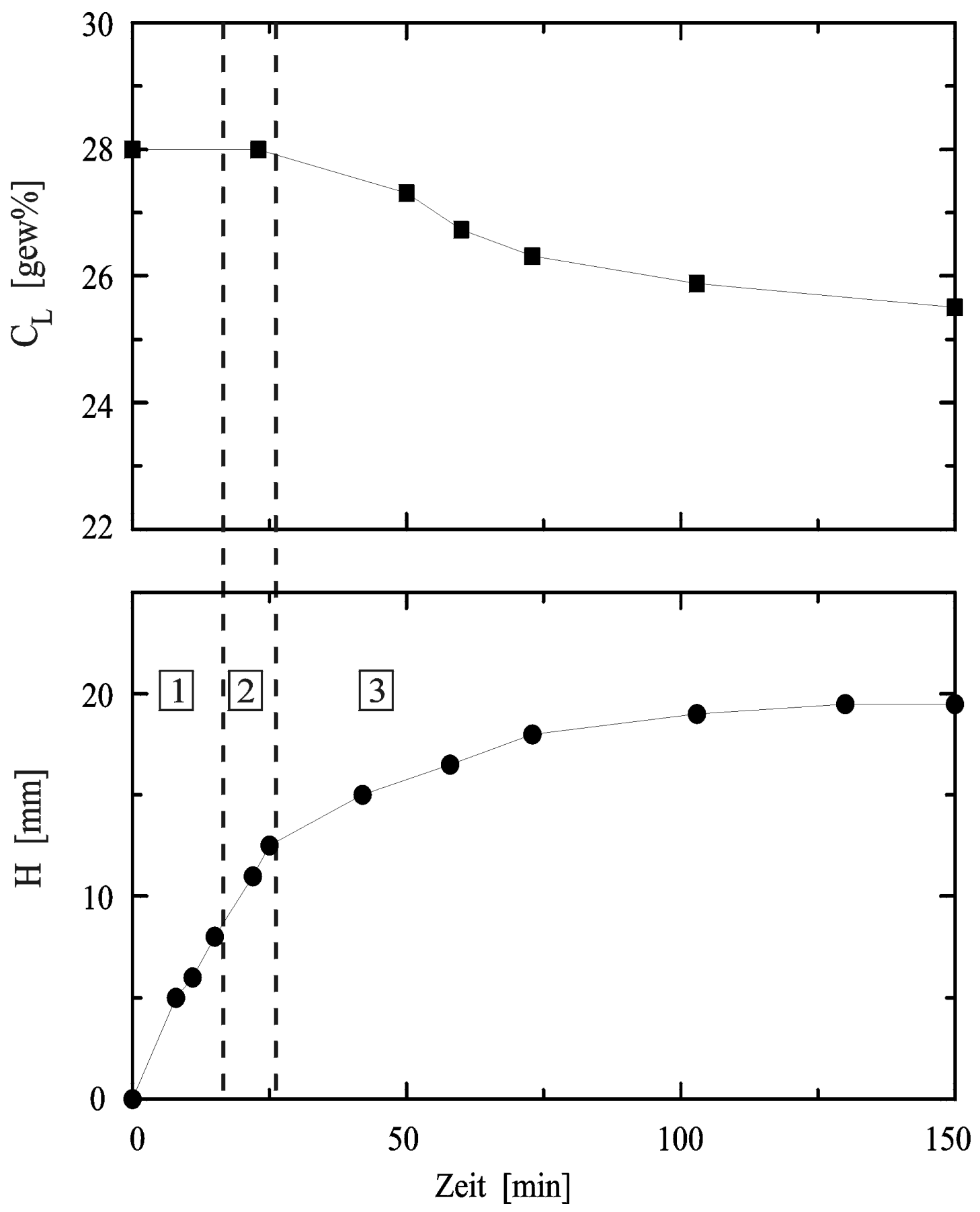

Abbildung 7.12: Konzentration

Gegenübergestellt werden die zeitliche Entwicklung der mittleren Kristallschichthöhe $H$ und der Konzentration $C_{L}$ in der flüssigen Phase. In die Abbildungen sind die unterschiedlichen Konvektionsmoden in den Bereichen 1-3 eingetragen. Im ersten Bereich kommt es zu Protoplumes und Fingerkonvektion, in Bereich 2 koexistieren Fingerkonvektion und Plumekonvektion und im Bereich 3 dominiert die vollentwickelte Plumekonvektion. $\left(\Delta T=20 \mathrm{~K}, C_{\circ}=28 \mathrm{gew} \%, \theta=3 \mathrm{~K}, \Omega=0 \frac{\mathrm{rad}}{\mathrm{s}}\right)$ 


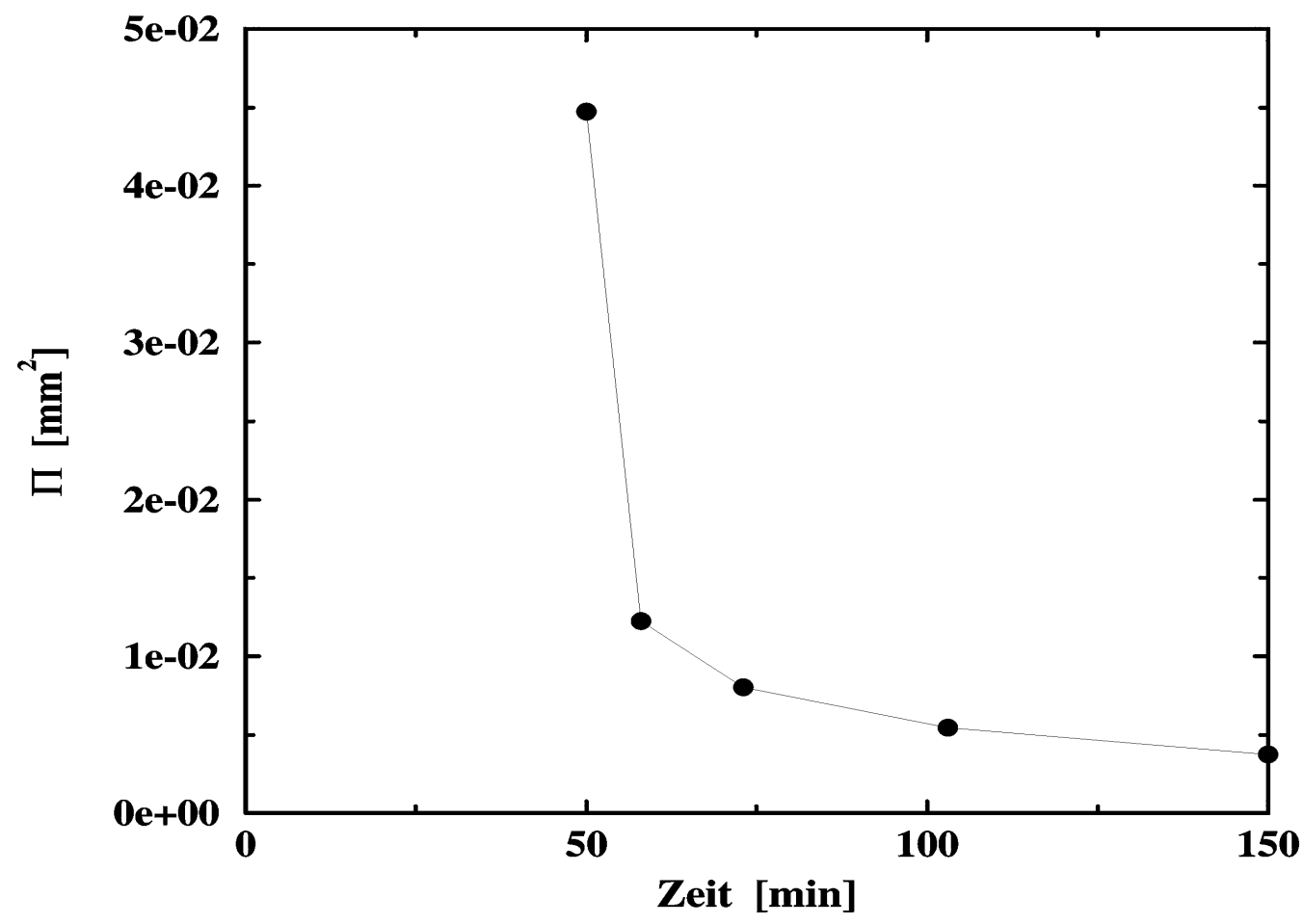

Abbildung 7.13: Permeabilität aus Konzentrationsmessung

Die Permeabilität $\Pi$ der Dendritenschicht wird mit den Konzentrationsmessungen aus Abbildung 7.19 bestimmt.

der Plumekonvektion. Der mittlere Dendritendurchmesser ist $d=0,4 \mathrm{~mm}$. Die Permeabilität der Dendritenschicht für den Einsatz der Plumekonvektion beträgt somit $\Pi=2,5 \cdot 10^{-3} \mathrm{~mm}^{2}$.

\subsubsection{Kamine}

Im Fall konstanter Rotation und gut abgestimmter Außentemperatur ist die Verteilung der Kamine in der Kristallschicht unregelmäßig, sie lassen kein signifikantes Muster er-

\begin{tabular}{|c|c|c|c|c|c|}
\hline$\Omega\left[\frac{\mathrm{rad}}{\mathrm{s}}\right]$ & $\mathrm{a}[\mathrm{mm}]$ & $\mathrm{b}[\mathrm{mm}]$ & $\phi$ & $\chi$ & $\Pi\left[\mathrm{mm}^{2}\right]$ \\
\hline \hline 0 & 0,09 & 0,185 & 0,237 & 0,763 & $1,42 \cdot 10^{-3}$ \\
\hline 2,1 & 0,085 & 0,205 & 0,175 & 0,828 & $3,08 \cdot 10^{-3}$ \\
\hline 6,3 & 0,105 & 0,175 & 0,360 & 0,640 & $0,40 \cdot 10^{-3}$ \\
\hline
\end{tabular}

Tabelle 7.2: Permeabilität und Porosität $\left(\Delta=24,5 \mathrm{~K}, C_{\circ}=26\right.$ gew $\left.\%\right)$ 
kennen. Vergleicht man Verteilung, Anzahl und Größe der Kamine in Experimenten gleicher Anfangskonzentration und vergleichbarer Bodentemperatur aber mit unterschiedlichen, konstanten Rotationsraten miteinander, findet man keine signifikanten Unterschiede. Abbildung 7.14 zeigt die Kaminverteilung eines Experimentes ohne Rotation (oben) und eines mit Rotation (unten, $\Omega=1,1 \frac{\mathrm{rad}}{\mathrm{s}}, \mathrm{E}=7,5 \cdot 10^{-5}$ ) bei gleichen Anfangsbedingungen. Die Kamine sind in beiden Fällen unregelmäßig ohne signifikantes Muster über die gesamte Fläche verteilt. Die Anzahl beträgt für den Versuch ohne Rotation 24 Stück und für das Vergleichsexperiment 29 Stück. Sie liegen damit in guter Übereinstimmung. Auch der Durchmesser ist mit etwa 2 mm gleich. In Kapitel 8.6 werden weiterführend Anzahl und Größe der Kamine beim Einsatz der Plumekonvektion sowie die zeitliche Entwicklung untersucht. Dies bestätigt die Ergebnisse der Experimente mit $\mathrm{NH}_{4} \mathrm{Cl}-\mathrm{H}_{2} \mathrm{O}$ von Neilson \& Incropera (1993b) und Sample \& Hellawell (1984), die ebenfalls keine Beeinflusssung der Kaminentstehung feststellen können, wenn der Tank um seine vertikale Achse mit einer konstanten Winkelgeschwindigkeit rotiert. 

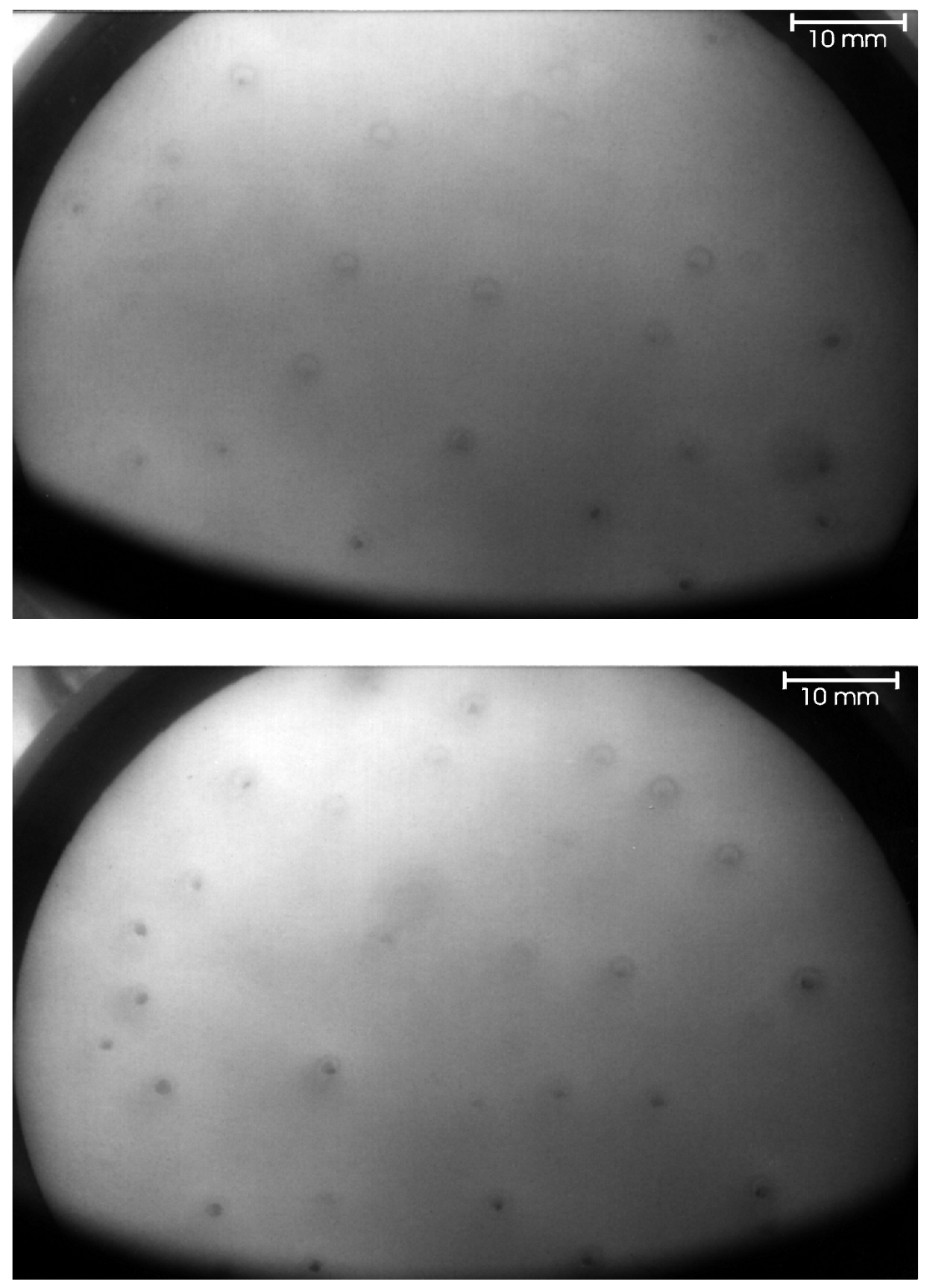

Abbildung 7.14: Kaminverteilung ohne Rotation (oben) und mit Rotation (unten)

Blick von oben auf die Kaminverteilung im zeitlich quasistabilen Zustand nach Einsatz der Plumekonvektion (180 min (oben) bzw. 205 min (unten)). Die Verteilung der Kamine über die Schicht ist in beiden Fällen unregelmäßig. Der Durchmesser der Kamine und ihre Anzahl ist ebenfalls vergleichbar.

(oben: $\Delta T=12 \mathrm{~K}, C_{\circ}=24 \mathrm{gew} \%, \theta=12,7 \mathrm{~K}, \Omega=0 \frac{\mathrm{rad}}{\mathrm{s}}$ )

(unten: $\Delta T=12 \mathrm{~K}, C_{\circ}=24 \mathrm{gew} \%, \theta=11,3 \mathrm{~K}, \Omega=1,1 \frac{\mathrm{rad}}{\mathrm{s}}, E=7,5 \cdot 10^{-5}$ ) 


\section{Kapitel 8}

\section{Ergebnisse: Konvektion}

Im folgenden Kapitel sollen die unterschiedlichen beobachteten Konvektionsmoden und deren Ursache beschrieben werden. Zunächst werden die verschiedenen Stadien der Konvektion des nicht rotierenden Systems dargestellt und der Einfluss der äußeren Parameter untersucht. Im zweiten Teil wird dann der Einfluss der Rotation untersucht, insbesondere ob neue Strömungsinstabilitäten entstehen.

\subsection{Protoplumes - Frühstadium der Konvektion}

Die Konvektion setzt zeitgleich mit der Bildung von Kristallen auf der Bodenplatte ein. Die Anfangsphase der Konvektion wird in der Literatur (z. B. Chen \& Chen, 1991 ( $\Delta T$ : 4-45 K), Tait \& Jaupart, $1992(\Delta T \sim 33 \mathrm{~K}))$ als Fingerkonvektion oder doppeltdiffusive Konvektion (Abb. 8.1) beschrieben, bei der die Bewegung in fingerförmigen kleinskaligen Auf- und Abströmen stattfindet. Dieses wird auch in den hier durchgeführten Experimenten bei einer Unterkühlung im Bereich von etwa $15 \mathrm{~K}$ bis $36 \mathrm{~K}$ beobachtet.

Bei kleiner Unterkühlung $(\Delta T<15 \mathrm{~K})$ tritt jedoch vor der Fingerkonvektion eine andere Strömungsinstabilität auf, die sich durch dünne, hoch aufsteigende Fäden äußert, die den später entstehenden Plumes sehr ähneln. Auf Grund dieser Analogie werden sie im Folgenden als „Protoplumes“ bezeichnet.

Abbildung 8.2 zeigt die Strömungsstruktur 11 min bzw. 21 min nach der Keimbildung für $\Delta T=12 \mathrm{~K}$. In diesem Bereich ist die Entstehung von Protokaminen (vgl. Kap. 7.2) sehr ausgeprägt, die Bodenplatte jedoch noch nicht vollständig bedeckt. Es existieren nur vereinzelte Kristallhäufchen (Pyramiden) mit einer maximalen Höhe von $2 \mathrm{~mm}$. 


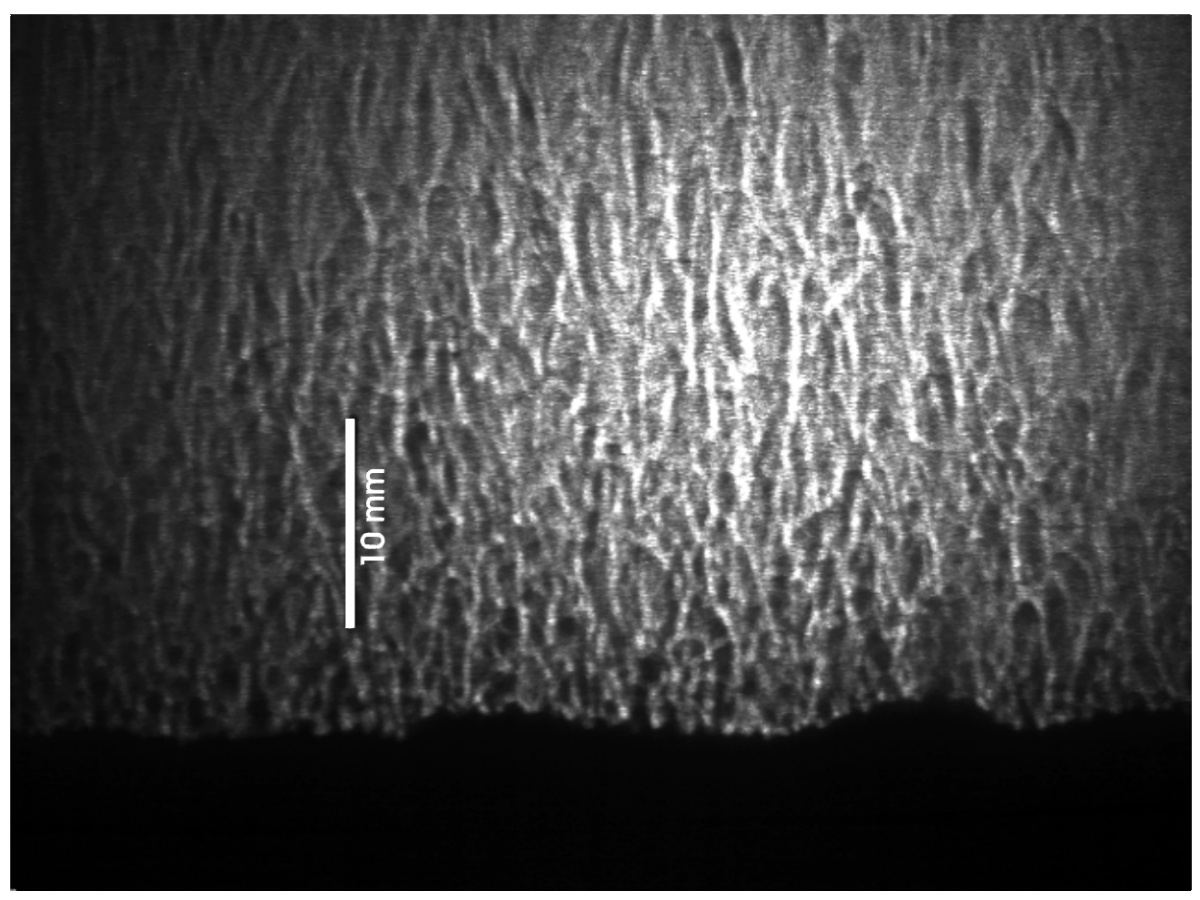

Abbildung 8.1: Fingerkonvektion

$\left(\Delta T=15 \mathrm{~K}, C_{\circ}=26 \mathrm{gew} \%, \theta=1,3 \mathrm{~K}, \Omega=4,2 \frac{\mathrm{rad}}{\mathrm{s}}\right)$
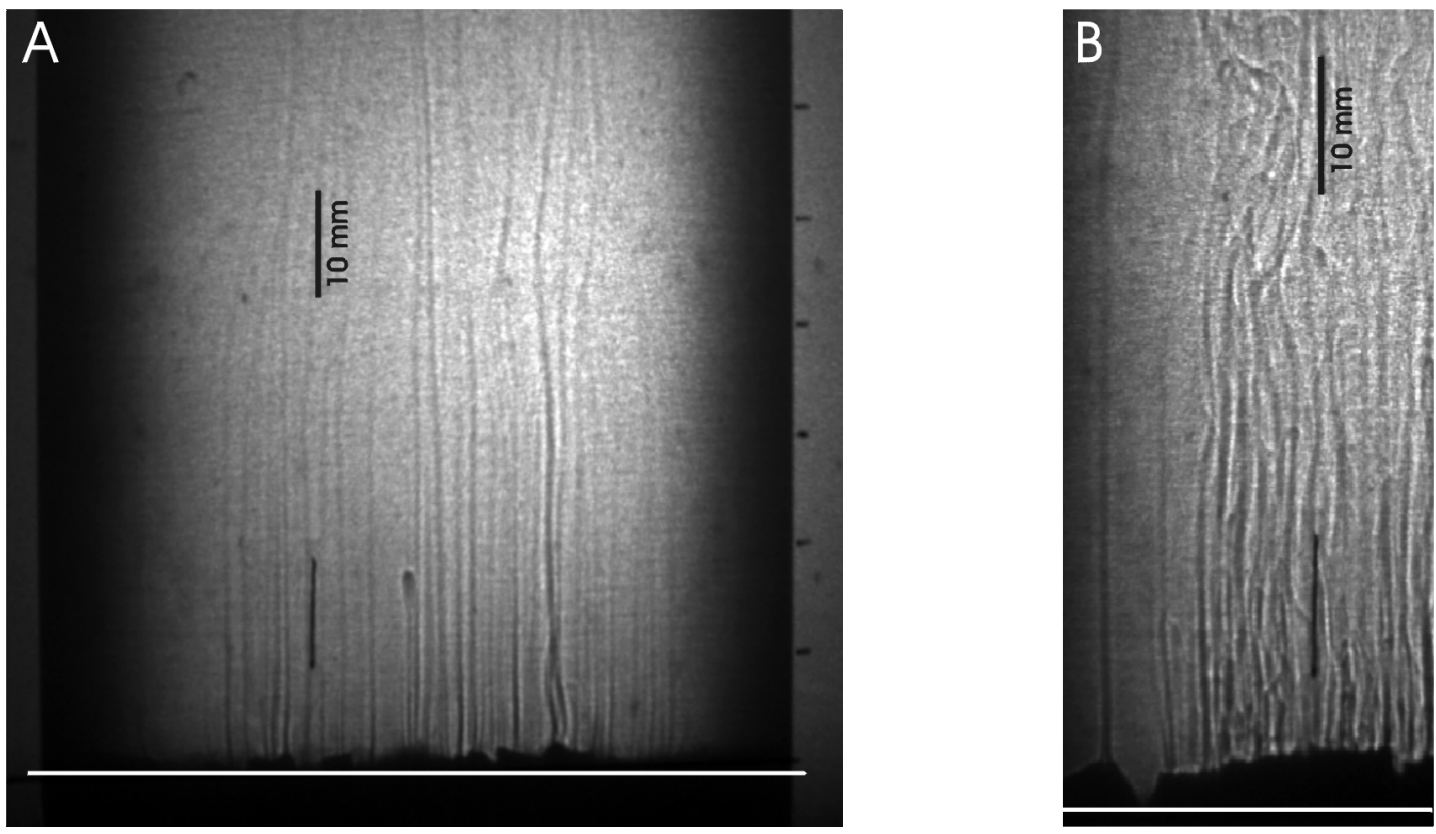

Abbildung 8.2: Protoplumes

Aus der Mitte der Kristallpyramiden, in denen sich Protokamine gebildet haben, steigen Protoplumes auf. Mit weißen Linien ist $H=0 \mathrm{~mm}$ gekennzeichnet. $A$ : $t=11 \mathrm{~min}$; $B: t=21 \min \left(C_{\circ}=26 \mathrm{gew} \%, \Delta T=12 \mathrm{~K}, \theta=0,9 \mathrm{~K}, \Omega=0 \frac{\mathrm{rad}}{\mathrm{s}}\right)$ 
Vergleicht man die Lage der späteren Protoplumes mit dieser frühen Kristallstruktur, so fälltt auf, dass die Protoplumes in der Mitte der Kristallkeime beginnen. An diesen Stellen kann deutlich ein Kamin beobachten werden. Nach weiteren 20 min hat sich eine geschlossene Fläche von Kristalliten gebildet, die Kamine sind mit Dendriten zugewachsen, und statt der Protoplumes kann im Schattenbild nur noch die kleinskalige Fingerkonvektion beobachtet werden.

Abbildung 8.2 B zeigt den Übergang zwischen den beschriebenen Stadien. In der Mitte und in der rechten Hälfte des Bildes hat sich die Schicht bereits weitestgehend geschlossen und die Protoplumes werden von stärker werdender Fingerkonvektion überlagert. Am äußeren linken Rand existiert noch ein vereinzelter Kristallberg, aus dessen Mitte ein Plume aufsteigt, doch im übrigen Bereich des Kristalls ist keine Konvektion zu beobachten. Die gesamte residuale Flüssigkeit wird also in diesem Fall durch den Protokamin in Form eines einzigen Protoplumes abgeführt.

\subsection{Fingerkonvektion}

In Experimenten mit höherer Unterkühlung $(\Delta T>15 \mathrm{~K})$ kann das Stadium der Protoplumes gar nicht oder nur schlecht beobachtet werden. Die Kristalle wachsen so schnell, dass die Bodenplatte schon nach wenigen Minuten (ca. 5-7 min) vollständig bedeckt ist. Die erste hier zu beobachtende Strömungsinstabilität ist die Fingerkonvektion. Abbildung 8.3 demonstriert den typischen zeitlichen Verlauf der Konvektion für $\Omega=0 \frac{\mathrm{rad}}{\mathrm{s}}$. Sie setzt ein, sobald die ersten Kristalle entstehen (Abb. 8.3 A) und hat eine vertikale Ausdehnung von 20 bis $30 \mathrm{~mm}$ oberhalb der Wachstumsfront. Die Bewegungen sind kleinskalig (Abb. 8.1) und turbulent. Als charakteristische Längenskala der Stömungsstruktur kann der Abstand zwischen den auf- und absteigenden Fingern mit etwa 0,5 bis $1 \mathrm{~mm}$ gelten. Die Strömungsgeschwindigkeit wird mit Hilfe von Videoaufnahmen auf 5-10 $\frac{\mathrm{mm}}{\mathrm{s}}$ abgeschätzt. Die Stärke der Konvektion nimmt mit dem Wachstum der Kristallschicht ab, bis die Fingerkonvektion schließlich von der Plumekonvektion abgelöst wird. Finger- und Plumekonvektion koexistieren für etwa 10 bis 20 min. Bei deren Einsatz (Abb. 8.3 B) beschränkt sich die Fingerkonvektion auf eine Höhe von etwa $10 \mathrm{~mm}$. 

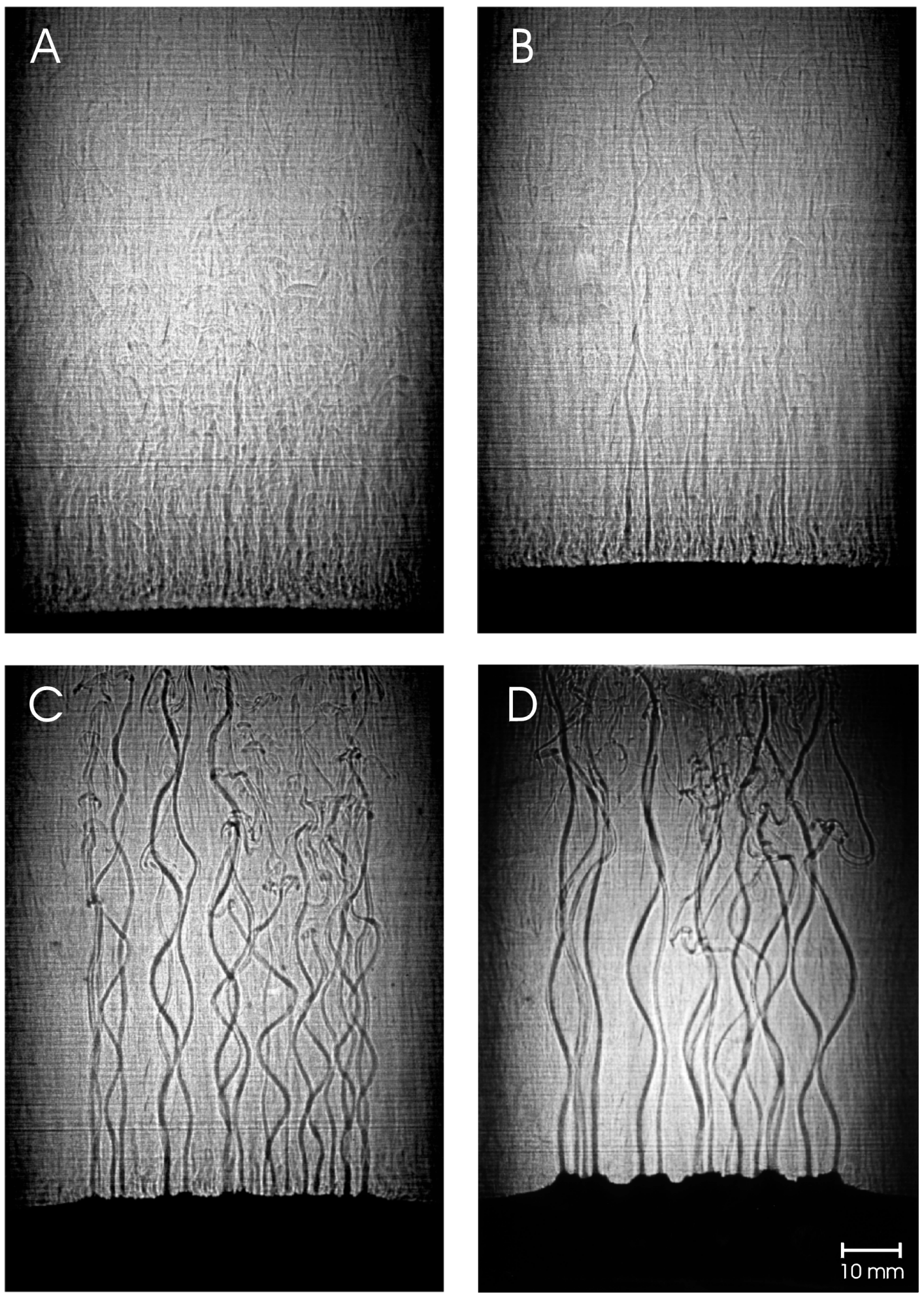

Abbildung 8.3: Schattenbilder der Konvektionsentwicklung ( $\left.\Omega=0 \frac{\mathrm{rad}}{\mathrm{s}}\right)$

A: Fingerkonvektion 9 min nach Keimbildung, B: Einsatz der Plumekonvektion bei einer Schichthöhe von $10 \mathrm{~mm}$ ( $t=37 \mathrm{~min})$, Koexistenz von Finger- und Plumekonvektion, $C$ : Plumekonvektion ( $t=65$ min), D: vollentwickelte Plumekonvektion ( $t=107$ min), $\left(C_{o}=26\right.$ gew $\%, \Delta T=35 \mathrm{~K}$ 


\subsection{Plumes}

Die dritte zu beobachtende Strömungsinstabilität ist die Plumekonvektion. Sie setzt nach der Fingerkonvektion ein, wenn sich in der porösen Schicht Kamine (siehe Abb. (7.6) ausgebildet haben. Plumes sind stark fokussierte, langgestreckte Aufströme, die aus den Kaminen in die flüssige Phase aufsteigen. Der Rückfluss findet über eine diffuse Strömung statt, die im Schattenbild nicht mehr aufgelöst werden kann.

In Abbildung $8.3 \mathrm{C}$ ist die Plumekonvektion voll entwickelt. Die Plumes haben bei Austritt aus dem Kamin eine Geschwindigkeit von 6-8 $\frac{\mathrm{mm}}{\mathrm{s}}$ und brechen z. T. bei 3/4 der Tankhöhe in kleine Flüssigkeitspakete auf. Im späteren zeitlichen Verlauf (Abb. 8.3 D) sinkt die Aufstiegsgeschwindigkeit auf 3-4 $\frac{\mathrm{mm}}{\mathrm{s}}$ und die Bewegung der Plumeschläuche wird ruhiger, so dass es seltener zu den kleinskaligen Instabilitäten kommt.

Ein Plume steigt zunächst geradlinig auf (Abb. 8.4). Nach etwa 10-20 mm wird er horizontal instabil und geht im zweidimensionalen Schattenbild in eine sinusförmige Welle über. Betrachtet man den Plume jedoch direkt von oben wird deutlich, dass der Plumeschlauch in einer langgestreckten Helix aufsteigt. Der Querschnitt der Helix schwankt

Abbildung 8.4: Plume-Größen

$H_{U}$ : Aufstiegshöhe pro Umlauf

$H_{V}$ : vertikale Aufstiegshöhe

R: Radius der Helix

$\alpha$ : Aufstiegswinkel

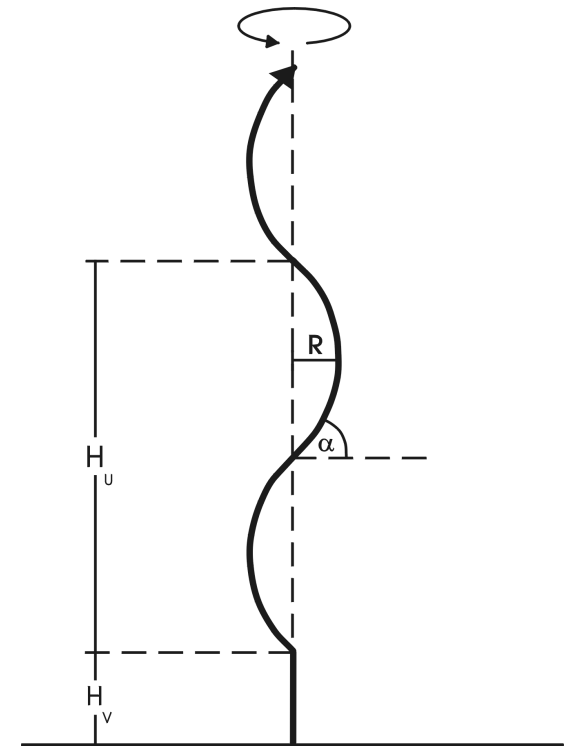

dabei zwischen kreisförmig und linear, so dass sowohl dreidimensionale als auch zweidimensionale Bewegungen auftreten, wobei die letzteren eher selten vorkommen. Mit dem Aufstieg vergrößert sich der Radius $R$ der Helix, während der Aufstiegswinkel $\alpha$, den der Plume mit der Horizontalen bildet, abnimmt. In der Nähe des Zylinderdeckels kann man oft beobachten, dass aus dem Plumestamm kleine Flüssigkeitströpfchen aus- 


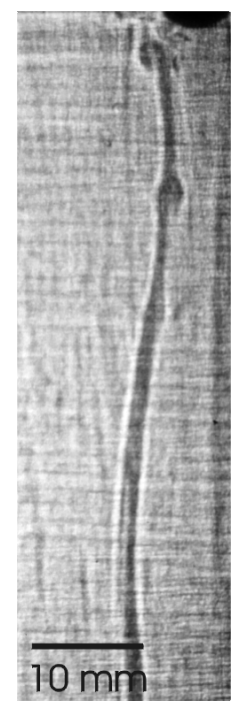

Abbildung 8.5: Lokale Ver-
dickungen im Plumestamm
Im oberen Drittel des Zylinders
kann es zu lokalen Verdickungen
im Plume kommen. Diese wan-
dern innerhalb des Plumes nach
oben.

brechen. Eine weitere Instabilität manifestiert sich in lokalen Verdickungen, die im Plume nach oben strömen (Abb. 8.5).

Hellawell et al. (1993) haben das horizontale Konzentrationsprofil durch den Plume durch eine Stufenfunktion angenähert (Abb. 8.6). Wie bereits in Kapitel 6 diskutiert, resultiert der Kontrast im Schattebild zum größten Teil aus der Konzentrationsdifferenz von Plume und Umgebungsflüssigkeit und nur zu einem geringen Anteil aus den thermischen Unterschieden. Somit lässt sich anhand des scharfen Helligkeitskontrasts der Plumedurchmesser ermitteln. Er schwankt zwischen 0,5 mm beim Einsatz der Konvektion und maximal 1,2 mm bei stärkerer Strömung. Hellawell et al. (1993) haben gezeigt, dass das Geschwindigkeitsprofil weit über diese engen Grenzen hinausreicht. Innerhalb des chemischen Plumes kann das Profil als parabolisch beschrieben werden, ausserhalb fällt es exponentiell ab. Das Einzugsgebiet eines Plumes ist durch die viskose Ankopplung mit der Umgebung etwa doppelt so breit wie der sichtbare Durchmesser im Schattenbild.

\subsection{Einsatz der Plumekonvektion}

Um den Einsatz der Plumekonvektion genau bestimmen zu können, sind eine Reihe modifizierter Experimente durchgeführt worden. Zu Beginn dieser Experimente wird, im Unterschied zu den bisherigen Versuchen, die Temperatur $T_{3}$ im äußeren Tank um 5-10 K über der Liquidustemperatur eingestellt $(\theta=5-10 \mathrm{~K})$. Im Verlauf des Experiments wächst dann die Kristallschicht nicht mehr ungestört bis in große Höhen an, sondern wird im Wachstum gebremst, sobald wärmere Regionen in der Flüssigkeit 


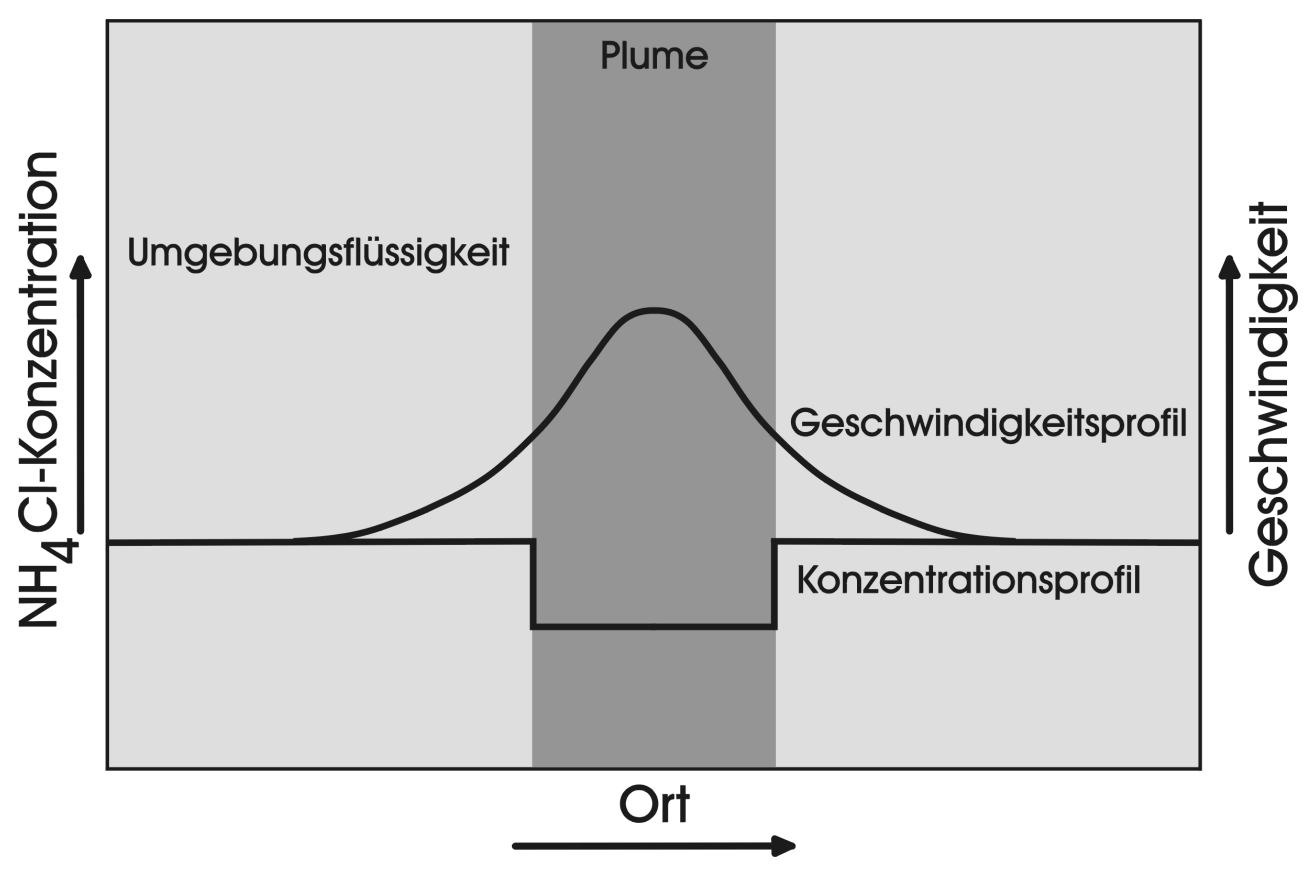

Abbildung 8.6: Konzentrations- und Geschwindigkeitsprofil eines Plumes

Das Konzentrationsprofil wird als eine Stufenfunktion mit steilen Flanken angenommen, deren Gradient den Helligkeitskontrast im Schattenbild bewirkt. Das Geschwindigkeitsprofil innerhalb des chemischen Plumes wird als parabolisch angenommen und fällt nach außen hin exponentiell ab.

erreicht werden. So lässt sich die Höhe der porösen Schicht, in der die ersten Kamine bzw. Plumes entstehen, genauer beobachten. Als Einsatz der Plumekonvektion wird hier der Zeitpunkt $t_{P}$ definiert, in dem der erste konzentrierte Aufstrom oberhalb der Fingerkonvektionszone im Schattenbild zu erkennen ist.

Es sind insgesamt 11 Versuche mit verschiedenen Anfangskonzentrationen $\left(C_{\circ}=24\right.$ gew\% und 26 gew\%), Temperaturdifferenzen $(\Delta T=4,5-24,5 \mathrm{~K})$ 円 und Winkelgeschwindigkeiten $\left(\Omega=0-6,3 \frac{\mathrm{rad}}{\mathrm{s}}\right)$ durchgeführt worden. Die maximale Schichthöhe in diesen Versuchen reicht von 7 bis maximal $25 \mathrm{~mm}$. In Experimenten, bei denen die Temperatur im Zylinder am Anfang nahe der Liquidustemperatur von $C_{\circ}$ gehalten wird $(\theta=0,2-2 \mathrm{~K})$, kann die Kristallschicht Höhen von bis zu $60 \mathrm{~mm}$ erreichen.

Abbildung 8.7 gibt eine Zusammenfassung der Ergebnisse, sowohl der speziell durchgeführten als auch aller anderen Versuche. Aufgetragen ist die Höhe $H_{P}=H\left(t=t_{P}\right)$, die die Kristallschicht bei Einsatz der Plumekonvektion erreicht hat in Abhängigkeit

\footnotetext{
${ }^{1}$ Bei $\Delta T=4,5 \mathrm{~K}$ kann mit einer maximalen Schichthöhe $H=7 \mathrm{~mm}$ kein Einsatz der Plumekonvektion beobachtet werden.
} 


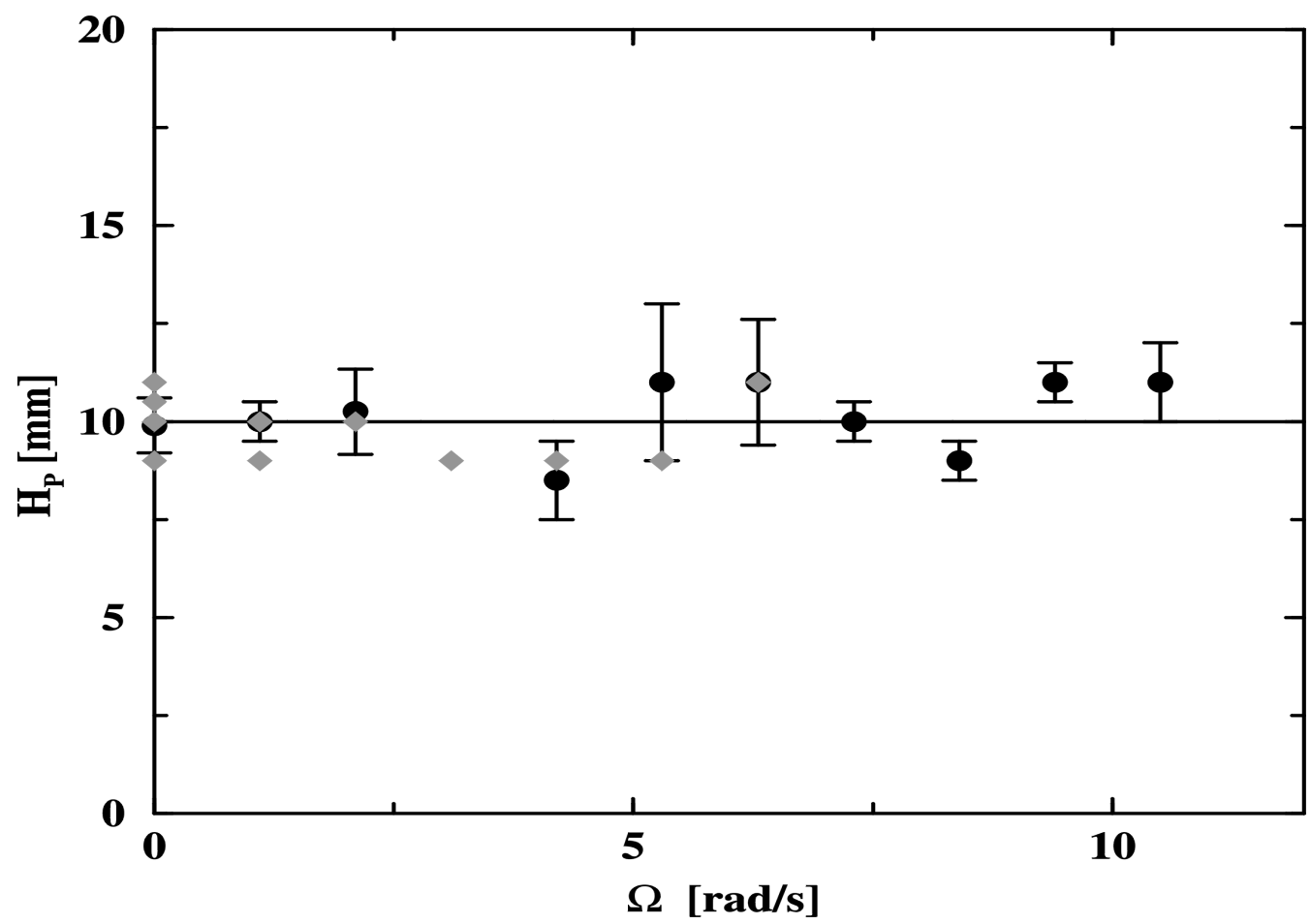

Abbildung 8.7: Höhe der Kristallschicht bei Einsatz der Plumeinstabilität Aus allen Experimenten mit gleicher Winkelgeschwindigkeit sind die Mittelwerte der Einsatzhöhen gebildet worden (gefüllte Kreise). Mit Rauten sind die Einzelergebnisse der speziellen Versuche für den Plumeeinsatz markiert.

von der Winkelgeschwindigkeit. Rauten kennzeichnen die Einzelergebnisse aus den oben beschriebenen Experimenten. Aus allen Versuchen mit gleichen Rotationsraten wurden die Mittelwerte der Einsatzhöhen gebildet. Sie sind mit gefüllten Punkten dargestellt. Die Fehlerbalken setzen sich aus den Standardabweichungen und der Messgenauigkeit von $1 \mathrm{~mm}$ zusammen. Die kritische Höhe der Dendritenschicht bei Einsatz der Plumekonvektion $H_{P}$ hat einen mittleren Wert von $H_{P}=10 \pm 1,3 \mathrm{~mm}$ und ist unabhängig von allen Versuchsparametern $\left(\Delta T, \theta, C_{\circ}\right.$ und $\left.\Omega\right)$.

Der Einsatz der Plumekonvektion hängt von der Strömung innerhalb der porösen Dendritenschicht ab. Aus Geichung 3.6 kann die kritische Rayleighzahl für den Einsatz bestimmt werden. Die Permeabilität beträgt $\Pi=2,5 \cdot 10^{-3} \mathrm{~mm}^{2}$ (s. Kap. 7.3.3) und $\Delta C \sim 0,5$ gew\% wird über den Brechungsindex von kleinen Proben bestimmt, die am Boden und kurz oberhalb der Schicht entnommen werden (vgl. Kap. 6.5). Setzt man die Werte für den Einsatz der Plumekonvektion ein, erhält man eine kritische Rayleighzahl $R_{m_{c}} \simeq 200$. Dieses Ergebnis stimmt mit Arbeiten von Chen \& Chen (1991) und Chen 
et al. (1994) überein, die jedoch den Aspekt der Rotation nicht berücksichtigt haben. Die Konvektion innerhalb der Schicht scheint also von der Rotation unbeeinflusst zu bleiben.

Um den Einfluss der Rotation abzuschätzen, kann eine modifizierte Ekmanzahl $E_{m}$ betrachtet werden. Sie gibt das relative Verhältnis von viskoser Reibung in der porösen Dendritenschicht (Darcy-Reibung) zur Corioliskraft an:

$$
E_{m}=\frac{\nu}{2 \Omega \Pi}
$$

Die Winkelgeschwindigkeit liegt in einem Bereich von $\Omega=0,5-10,5 \frac{\mathrm{rad}}{\mathrm{s}}$. Man erhält somit $E_{m}=24-500$. Daraus resultiert, dass der Einfluss der viskosen Kräfte um Größenordnungen grösser ist als der der Corioliskräfte. Es ist also, in Übereinstimmung mit dem Experiment, kein signifikanter Einfluss der Rotation auf die Konvektion innerhalb der porösen Schicht zu erwarten.

\subsection{Plumeanzahl beim Einsatz der Konvektion}

Die Anzahl der aktiven Kamine in der Schicht bzw. die Anzahl der Plumes $N_{P_{\circ}}$ bei Einsatz der Plumekonvektion variiert mit $\Delta T, \theta$ und $\Omega$. In nicht rotierenden Versuchen liegt $N_{P_{\circ}}$ typischerweise zwischen 10 und 20, kann jedoch bis auf 45 ansteigen. Tendentiell steigt die Anzahl bei konstanter Unterkühlung näherungsweise linear mit $\theta$ an (Abb. $8.8 \mathrm{~A}$ ). Wird die Unterkühlung $\Delta T$ erhöht, erhält man ebenfalls eine Zunahme von Kaminen.

Corioliskräfte beeinträchtigen die Anzahl deutlich. Abbildung 8.8 B zeigt die Abhängigkeit der Kaminanzahl von der Rotationsrate $(\Delta T \sim 24 \mathrm{~K} ; 0,2 \leq \theta \leq 1,9 \mathrm{~K})$. Ohne Rotation entstehen beim Einsatz 20 aktive Kamine. Bei $\Omega \neq 0 \frac{\mathrm{rad}}{\mathrm{s}}$ sinkt die Anzahl exponentiell mit steigendem $\Omega$ und hat für $\Omega=2.1 \frac{\mathrm{rad}}{\mathrm{s}}$ auf nur noch 2 bis 4 Plumes abgenommen. Mit zunehmendem $\Omega$ bleibt $N_{P_{\circ}}$ weiterhin sehr gering und schwankt zwischen 1 und 3.

\subsection{Zeitliche Entwicklung der Plumekonvektion}

Zu Beginn der Konvektion hängt die Plumeanzahl $N_{P}$, wie im vorherigen Kapitel 8.5 gezeigt, unter anderem von der Unterkühlung des Experimentes ab. Dieser Zusammenhang zeigt sich noch deutlicher im weiteren zeitlichen Verlauf. 

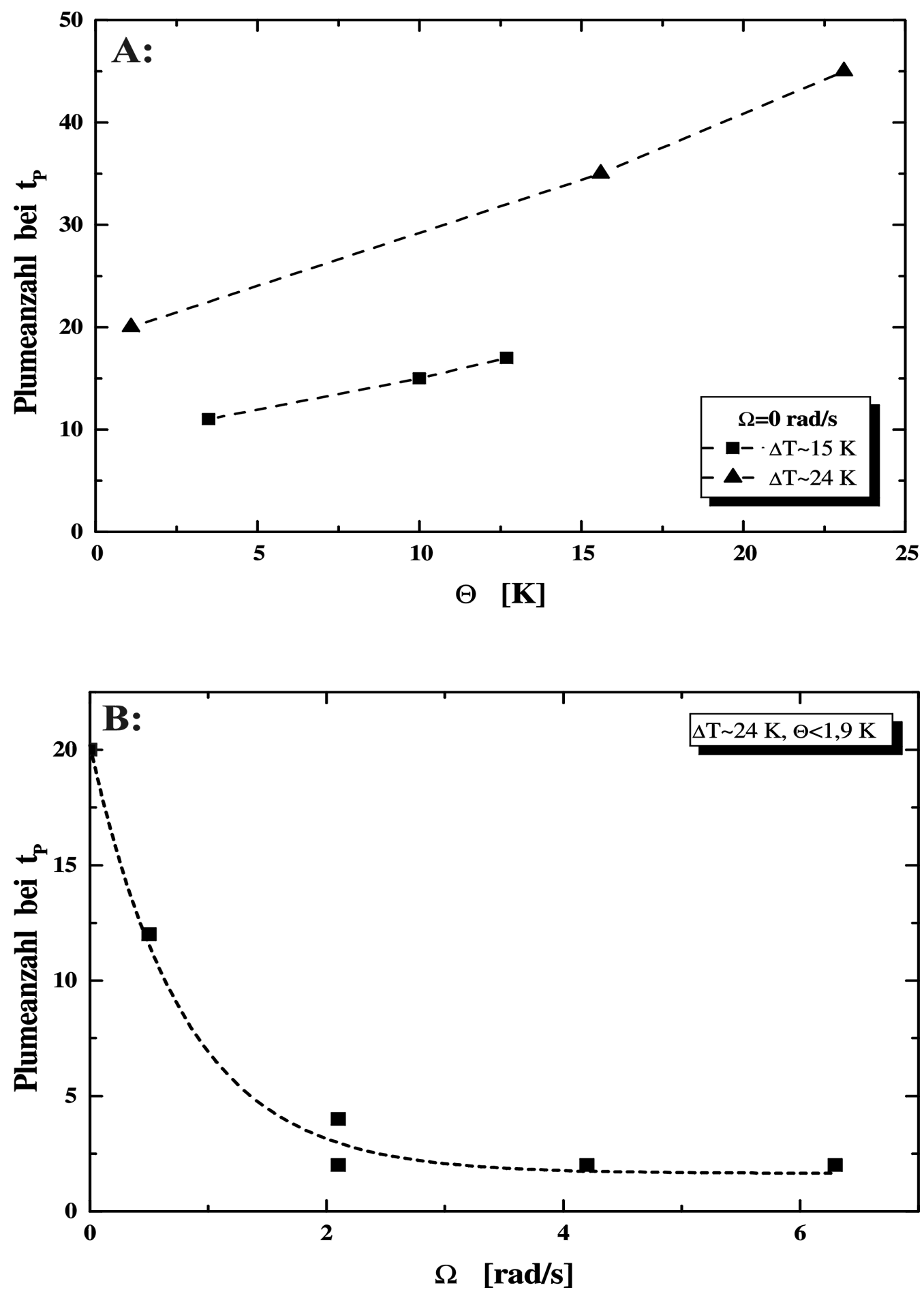

Abbildung 8.8: Plumeanzahl bei Konvektionseinsatz

A: Plumeanzahl steigt mit Erhöhung von $\Delta T$ und $\theta$.

B: Exponentieller Abfall der Plumeanzahl mit steigender Winkelgeschwindigkeit $\Omega$. 

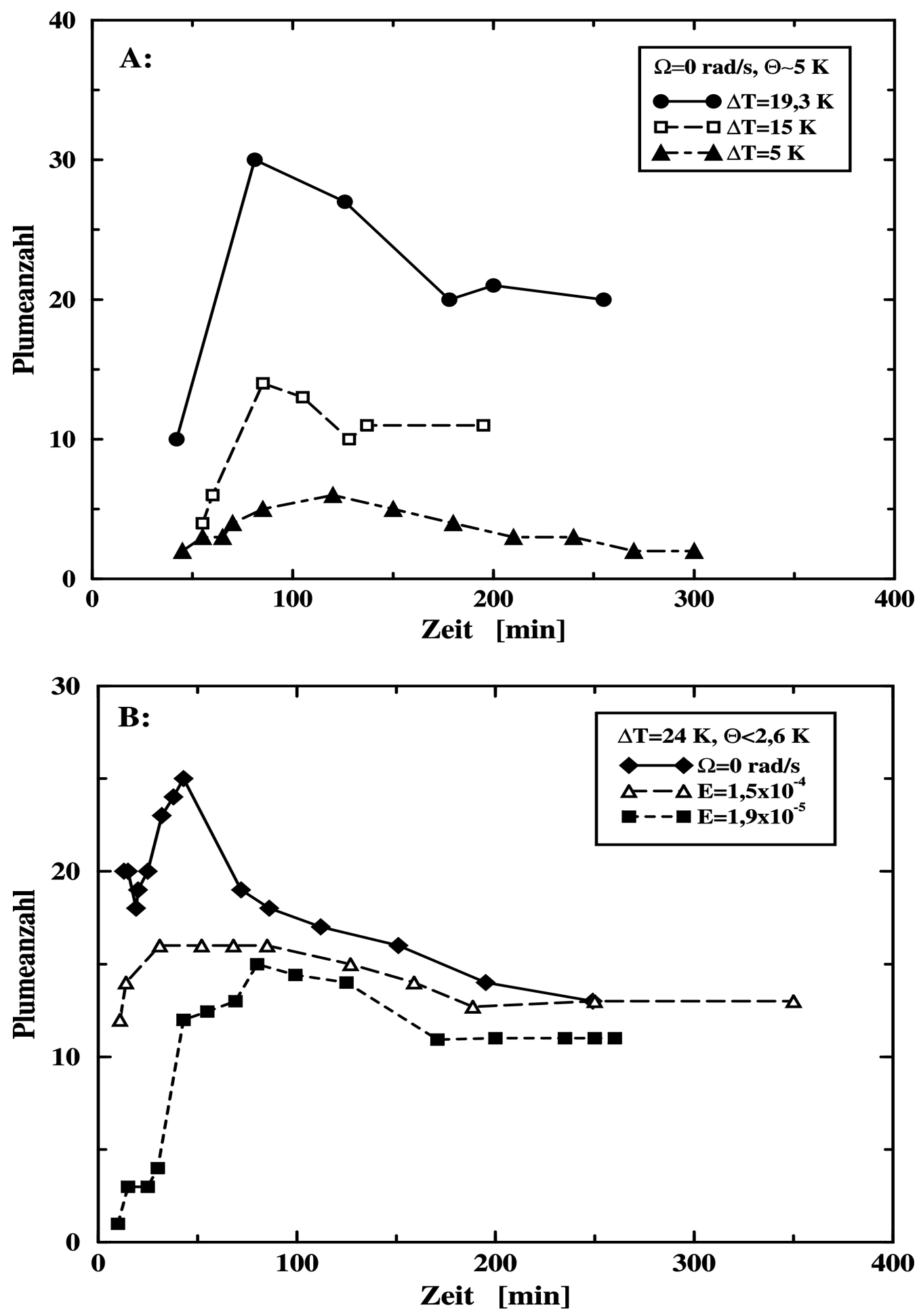

Abbildung 8.9: Zeitliche Entwicklung der Plumekonvektion

A: Plumeanzahl in Abhängigkeit der Unterkühlung $\Delta T$.

B: Plumeanzahl in Abhängigkeit der Rotationsrate bzw. der Ekmanzahl E. 
In Abbildung $8.9 \mathrm{~A}$ ist die zeitliche Entwicklung der Plumeanzahl für $\Omega=0, \theta \sim 5 \mathrm{~K}$ und $\Delta T: 5 \mathrm{~K}, 15 \mathrm{~K}$ und 19,3 K dargestellt. Die Plumekonvektion setzt bei allen Versuchen etwa zum gleichen Zeitpunkt ein, jedoch mit einer unterschiedlichen Anzahl $N_{P}$, wobei der Unterschied zwischen $\Delta T=5 \mathrm{~K}$ und $15 \mathrm{~K}$ nur minimal ist. Nach dem Einsatz steigt $N_{P}$ steil an, bis eine maximale Plumeanzahl erreicht wird. Die Steigung des Anstiegs nimmt dabei deutlich mit der Unterkühlung zu. Bei $\Delta T=15 \mathrm{~K}$ und 19,3 $\mathrm{K}$ sinkt $N_{P}$ mit etwa der gleichen Steigung wieder relativ schnell ab, bis ein etwa konstanter Wert für mehrere Stunden erreicht wird.

Die Entwicklung bei $\Delta T=5 \mathrm{~K}$ ist gleichförmiger, mit einem leichten anfänglichen Anstieg und einem sehr langsamen Abfall. Die Werte für $N_{P}$ steigen mit Erhöhung der Unterkühlung, da eine hohe Unterkühlung einen schnellen Kristallisationsprozess zur Folge hat und damit einen erhöhten Massenfluss.

Der oben beschriebene prinzipielle Verlauf der Plumeentwicklung — rascher Anstieg und sanfter Abfall — bleibt auch unter dem Einfluss der Rotation erhalten. Die Tendenz, dass weniger aktive Kamine beim Einsatz der Konvektion beteiligt sind (Kap. 8.5), setzt sich in der zeitlichen Entwicklung fort. Abbildung 8.9 B zeigt dies für drei unterschiedliche Winkelgeschwindigkeiten $\left(\Omega\right.$ : $0 ; 0,5$ und $4,2 \frac{\mathrm{rad}}{\mathrm{s}}$; bzw. $E: \infty ; 1,5 \cdot 10^{-4}$ und $\left.1,9 \cdot 10^{-5}\right)$

Die Konvektion setzt bei allen Versuchen etwa zum gleichen Zeitpunkt ein. Die Steigungen während des Anstiegs unterscheiden sich stark voneinander, zeigen aber keinen Zusammenhang mit der Rotationsrate. Nach einem kurzzeitigen Maximum sinkt in allen Fällen die Anzahl wieder ab, bis sich ein quasi-stationärer Zustand eingestellt hat, bei dem $N_{P}$ nur noch wenig variiert und für alle $\Omega$ etwa gleich ist. Die Rotation scheint also einen starken Einfluss auf das Anfangsstadium der Plumekonvektion zu haben, wohingegen die Dynamik des vollentwickelten Systems eher von der Unterkühlung beeinflusst wird.

\subsection{Einfluss der Rotation auf die Strömung}

In den vorangegangenen Kapiteln wurde gezeigt, dass die Rotation einen zu vernachlässigenden Einfluss auf das Kristallwachstum hat, wohingegen die Stärke der Konvektion bzw. die Plumeanzahl beeinträchtigt wird. Im Folgenden soll nun die Strömungsstruktur für $\Omega \neq 0 \frac{\mathrm{rad}}{\mathrm{s}}$ genauer untersucht werden. 


\subsubsection{Zeitliche Entwicklung der Konvektion}

Der prinzipielle zeitliche Verlauf der Konvektion, wie er in Kapitel 8.2 und 8.3 beschrieben ist, bleibt im rotierenden System unverändert. In Abbildung 8.10 wird dies exemplarisch dargestellt. Sofort nach der Keimbildung setzt die Fingerkonvektion (Abb. 8.10 A) ein. Beim Erreichen der kritischen Schichthöhe (hier: $H=9,5 \mathrm{~mm}$ ) kann im Schattenbild ein vereinzelter Plume beobachtet werden (Abb. 8.10 B, linker Bildrand). Die Plumeanzahl nimmt danach stark zu, wie bereits in Kapitel 8.6 erläutert. Nach etwa zwei Stunden ist in diesem Experiment eine maximale Anzahl von $N_{P}=13$ erreicht (Abb. $8.10 \mathrm{C}$ ). In Abbildung $8.10 \mathrm{D}$ ist die Plumekonvektion bereits weit fortgeschritten im quasi-stationären Zustand.

Man sieht, dass die Fingerkonvektion von der Rotation nicht beeinträchtigt wird, die Plumekonvektion jedoch stark beeinflusst wird. Im nächsten Kapitel wird dies qualitativ untersucht.

\subsubsection{Tröpfcheninstabilität}

Im Regime der Plumekonvektion tritt eine neue Instabilität auf. In Abbildung 8.11] sieht man den Aufstieg eines Plumes bei $\Omega=2,1 \frac{\mathrm{rad}}{\mathrm{s}}$. Er steigt nur eine kurze Strecke vertikal nach oben und wird, wie im Fall $\Omega=0 \frac{\mathrm{rad}}{\mathrm{s}}$, helikal instabil. Der Aufstiegswinkel wird jedoch wesentlich flacher und erreicht mit etwa $65^{\circ}$ einen überkritischen Wert. Der Plume ist dann instabil in seiner Umgebungsflüssigkeit geschichtet, da er eine geringere Dichte $\rho_{P}$ als die Umgebung mit $\rho_{L}$ hat. Er bricht in eine Vielzahl kleiner Flüssigkeitströpfchen auf, die dann meist vollständig losgelöst vom Plumestamm aufsteigen. Dieser Prozess wird in Skizze 8.12 verdeutlicht.

Die äussere Form der Tröpfcheninstabilität erinnert an die pilzkopfartigen Diapire bzw. Ringwirbel, die sich beim Aufstieg von leichterem Material in einem dichteren Medium ausbilden (siehe z. B. Batchelor (1994), Tafel 20). In einigen der hier durchgeführten Versuche erkennt man nur den Kopf des Tropfens, in anderen Fällen wird noch ein kleiner Schwanz nachgezogen. Die Tropfen steigen nahezu senkrecht auf, wobei ihr Schattenbild zunehmend verblasst und schließlich nicht mehr auszumachen ist. Dieses Verschwinden ist auf chemische Diffusion zurückzuführen, da, wie bereits erwähnt, die thermische Diffusivität sehr groß ist und der Plume darum bei Ablösung der Tröpfchen bereits Umgebungstemperatur haben dürfte. Analog zur thermischen Diffusions- 

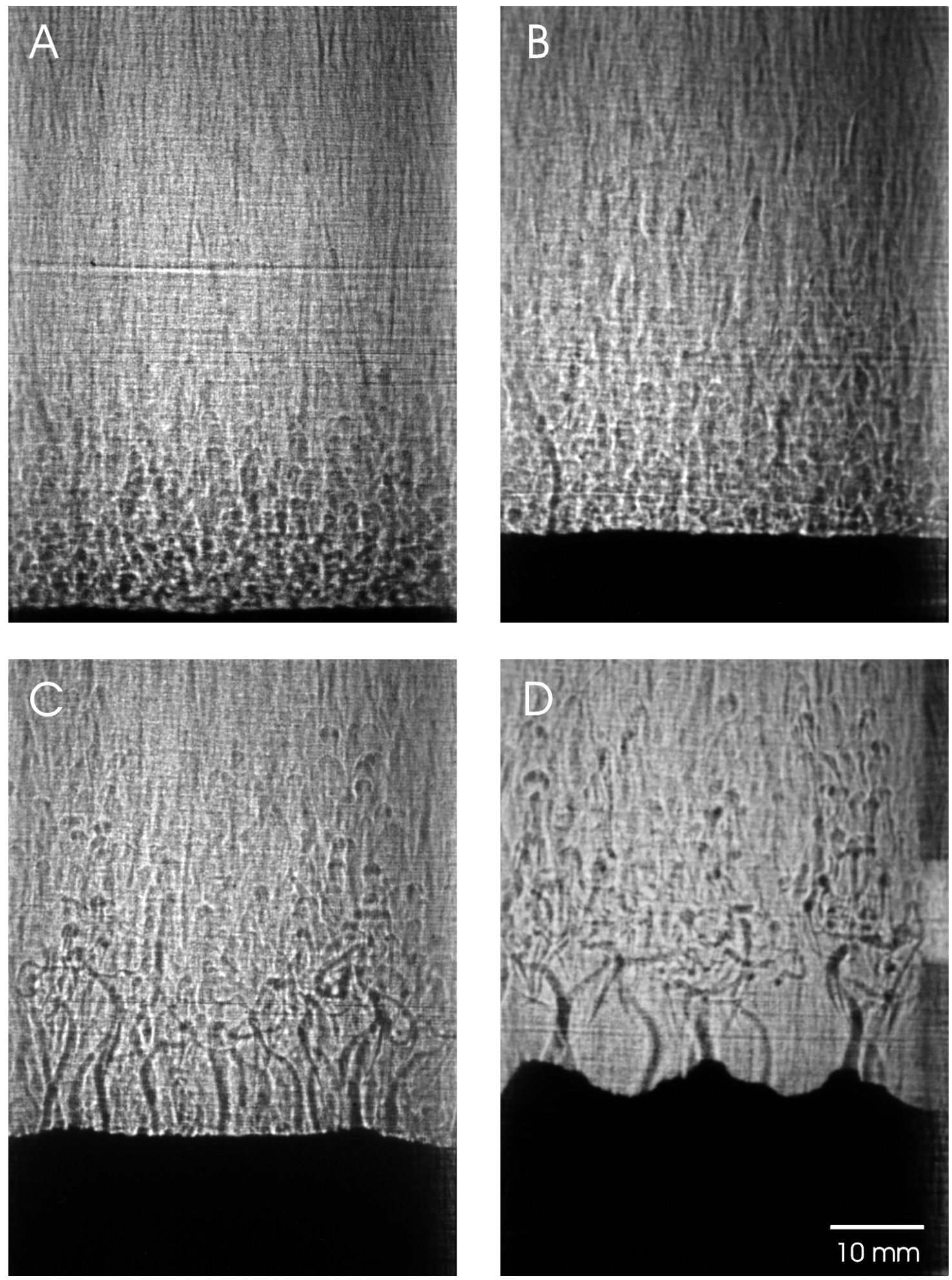

Abbildung 8.10: Schattenbilder der Konvektionsentwicklung $\left(\Omega=5,2 \frac{\mathrm{rad}}{\mathrm{s}}\right)$ A: Fingerkonvektion 9 min nach Keimbildung, B: Einsatz der Plumekonvektion bei einer Schichthöhe von 9,5 mm mit einem Plume ( $t=54$ min), Koexistenz von Fingerund Plumekonvektion, $C$ : Plumekonvektion ( $t=118$ min, $\left.N_{P}=13\right)$, D: vollentwickelte Plumekonvektion ( $\left.t=220 \mathrm{~min}, N_{P}=8\right),\left(C_{o}=24\right.$ gew $\left.\%, \Delta T=15 \mathrm{~K}, \theta=10 \mathrm{~K}\right)$ 


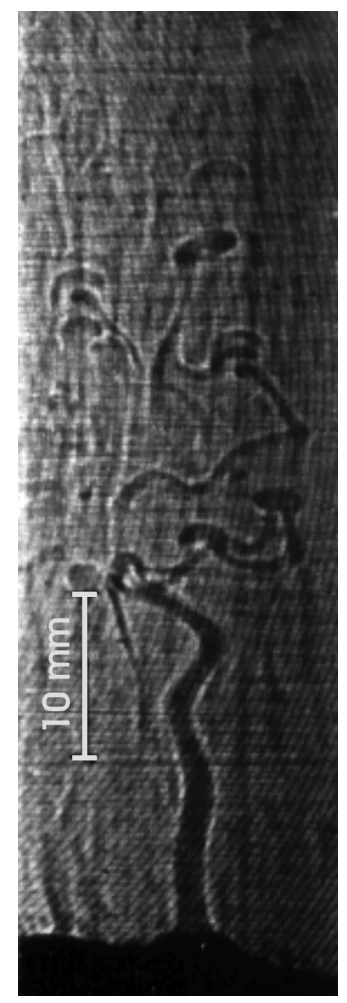

\begin{abstract}
Abbildung 8.11: Aufbrechender Plume
Der Plume steigt zunächst vertikal in der flüssigen Phase auf, wird dann horizontal instabil und beginnt in eine Helix überzugehen. Der Aufstiegswinkel der Helix neigt sich jedoch ab einer Höhe $H_{\text {Inst }}$ so stark, dass er in kleine Flüssigkeitspakete aufbricht $\left(C_{\circ}=24\right.$ gew $\%$, $\Delta T=15 K, \theta=4 K, \Omega=2,1 \frac{\mathrm{rad}}{\mathrm{s}}, E=$ $\left.3,8 \cdot 10^{-5}\right)$.
\end{abstract}

zeit (Gl. 5.1) läßt sich eine chemische Diffusionszeit $t_{C}$ definieren:

$$
t_{C}=\frac{L^{2}}{\kappa_{C}}
$$

Die chemische Diffusionskonstante $\kappa_{C}$ beträgt $1,7 \cdot 10^{-3} \frac{\mathrm{mm}^{2}}{\mathrm{~s}}$. Nimmt man als Längenskala $L$ den Radius des Tröpfchens an, der bei der Ablösung des Tropfens vom Plumestamm typischerweise $1 \mathrm{~mm}$ beträgt und auf bis zu $2 \mathrm{~mm}$ ansteigt, kurz bevor das Tröpfchen nicht mehr im Schattenbild zu erkennen ist, so ergibt sich eine Diffusionszeit von $t_{C} \approx 10 \mathrm{~min}$. Dem gegenüber steht die viel kürzere Zeit, in der Größenordnung von etwa $20 \mathrm{~s}$, in denen sich die Tröpfchen beobachten lassen. (In dieser Zeit steigt ein Tropfen mit einer mittleren Geschwindigkeit von $2 \frac{\mathrm{mm}}{\mathrm{s}}$ in typische Höhen von 30-50 mm.) Diese Diskrepanz lässt sich nicht allein damit erklären, dass in der Schattenbildprojektion nicht beliebig kleine Dichtekontraste aufgelöst werden können und auch nicht dadurch, dass der sichtbare Tropfenradius nur ungenügend den tatsächlichen Dichtegradienten wiedergibt.

Geht man dagegen davon aus, dass die Tröpfchen kleine Ringwirbel sind, wie die Beobachtungen vermuten lassen, dann bestehen sie aus „,aufgewickelten“ dünnen Schichten von ursprünglichem Plumematerial und beim Aufstieg mitgerissenem Umgebungsmaterial (Abb. 8.14). Dieser Vorgang lässt die Tröpfchen anwachsen, wobei sich jedoch die 
Abbildung 8.12: Entstehung der Tröpfcheninstabilität

Einzelne Flüssigkeitspakete lösen sich von einem überkritisch geneigten primären Plumeschlauch $a b$ und steigen nach oben.
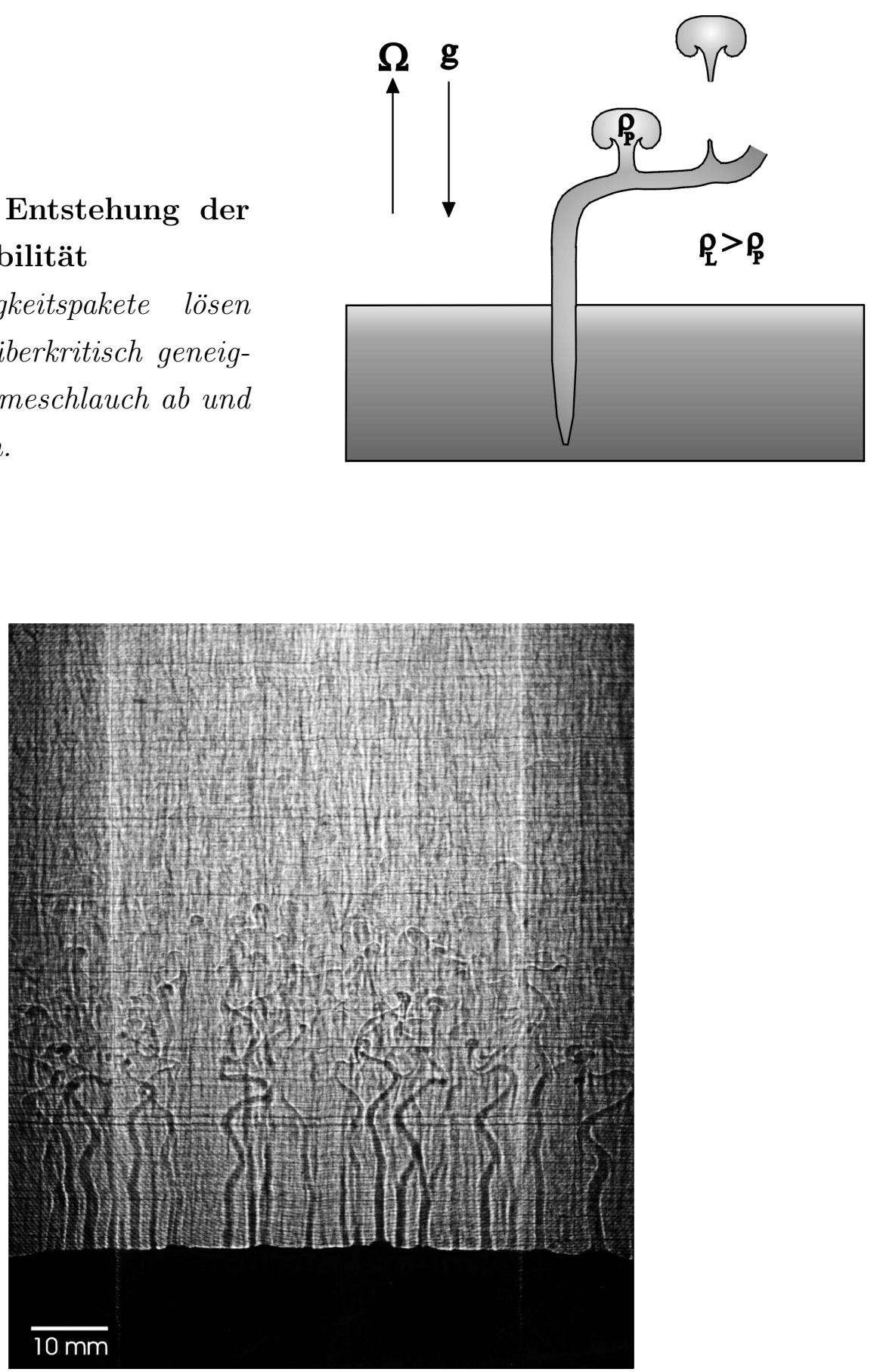

Abbildung 8.13: Tröpfcheninstabilität bei $\Omega=1,1 \frac{\mathrm{rad}}{\mathrm{s}}$

Die vom Plumestamm losgelösten Tröpfchen haben sich nach einer Höhe von 20 bis $30 \mathrm{~mm}$ weitgehend an ihre Umgebung angepasst, so dass sie im Schattenbild kaum noch zu erkennen sind $\left(C_{\circ}=24\right.$ gew $\left.\%, \Delta T=12 K, \theta=11 K, E=7,5 \cdot 10^{-5}\right)$. 


\section{Abbildung 8.14: Diapir}

Beim Aufstieg reißt das Tröpfchen Umgebungsmaterial mit. Dadurch nimmt zwar sein äußerer Durchmesser $D$ zu, die effektive Dicke $L$ der Flüssigkeitsschichten, die am Diffusionsprozess beteiligt sind, sinkt jedoch.

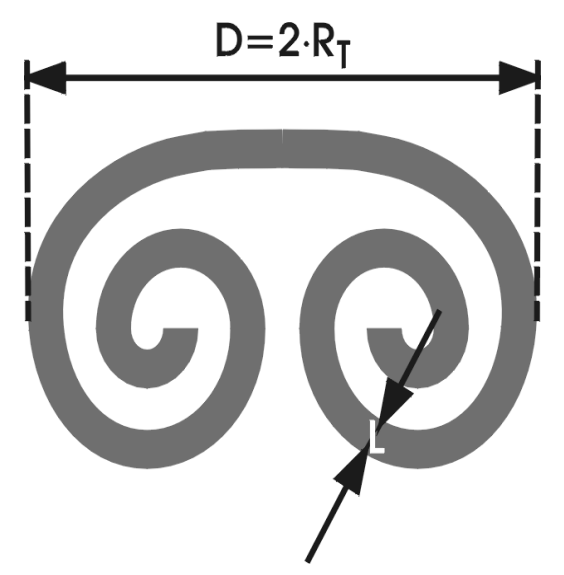

Dicke der einzelnen Schichten verringert. Diese Schichtdicke $L$ ist daher eine adäquatere Längenskala für den Diffusionsprozess als der Tröpfchenradius. Bei einer geschätzten Dicke der einzelnen Schichten von 0,5 mm, kurz nach der Loslösung vom primären Plumestamm, und 0,1 mm nach weiteren $20 \mathrm{~mm}$ Aufstiegshöhe, ergeben sich Diffusionszeiten von $5 \mathrm{~s}$ bis $150 \mathrm{~s}$, die deutlich besser mit den gemessenen Werten übereinstimmen.

\subsubsection{Die Natur der Tröpfcheninstabilität}

Diese neue Instabilität hat die Form der bekannten Rayleigh-Taylor Instabilität, wie sie in instabil geschichteten Flüssigkeitslagen auftritt. Um die Ursache der Instabilität zu prüfen, wird der Kontainer plötzlich in Rotation versetzt und die Auswirkungen auf einen ursprünglich nicht-rotierenden Plume untersucht (Abb. 8.15). Die Scherströme lenken den Plume um einem Winkel $\alpha$ von etwa $80^{\circ}$ von der Horizontalen ab. Da der Plume von geringerer Dichte ist als seine Umgebung, bricht er in eine Vielzahl kleiner Tröpfchen mit Durchmessern von etwa $2 \mathrm{~mm}$ auf. Whitehead (1982) hat in Laborexperimenten gezeigt, dass der kritische Aufstiegswinkel $\alpha_{C}$ für den Einsatz dieser Instabilität von der Viskosität und der Reynoldszahl $\left(R e=\frac{v \cdot r}{\nu}\right)$ abhängt. Bei einer Viskosität $\nu$ von $1 \cdot 10^{-2} \frac{\mathrm{cm}^{2}}{\mathrm{~s}}$ und $R e=0,3--40$ liegt $\alpha_{C}$ zwischen $20^{\circ}$ und $60^{\circ}$. Die mittlere Geschwindigkeit $v$ eines Plumes beträgt 0,7 $\frac{\mathrm{cm}}{\mathrm{s}}$ und der mittlere Plumeradius $r$ ist 0,07 cm. Die Reynoldszahl lässt sich damit auf 5 abschätzen. Für diesen Wert gibt Whitehead einen kritischen Winkel von $\alpha_{C} \approx 50^{\circ}$ an. Dieser Wert wird in allen Versuchen überschritten.

Die Bestimmung von $\alpha_{C}$ gestaltet sich in den weiter oben vorgestellten Versuchen mit einer konstanten Winkelgeschwingigkeit deutlich schwieriger. Der Plume ändert seinen Aufstiegswinkel in den meisten Fällen nicht stetig, sondern neigt sich plötzlich, sobald 


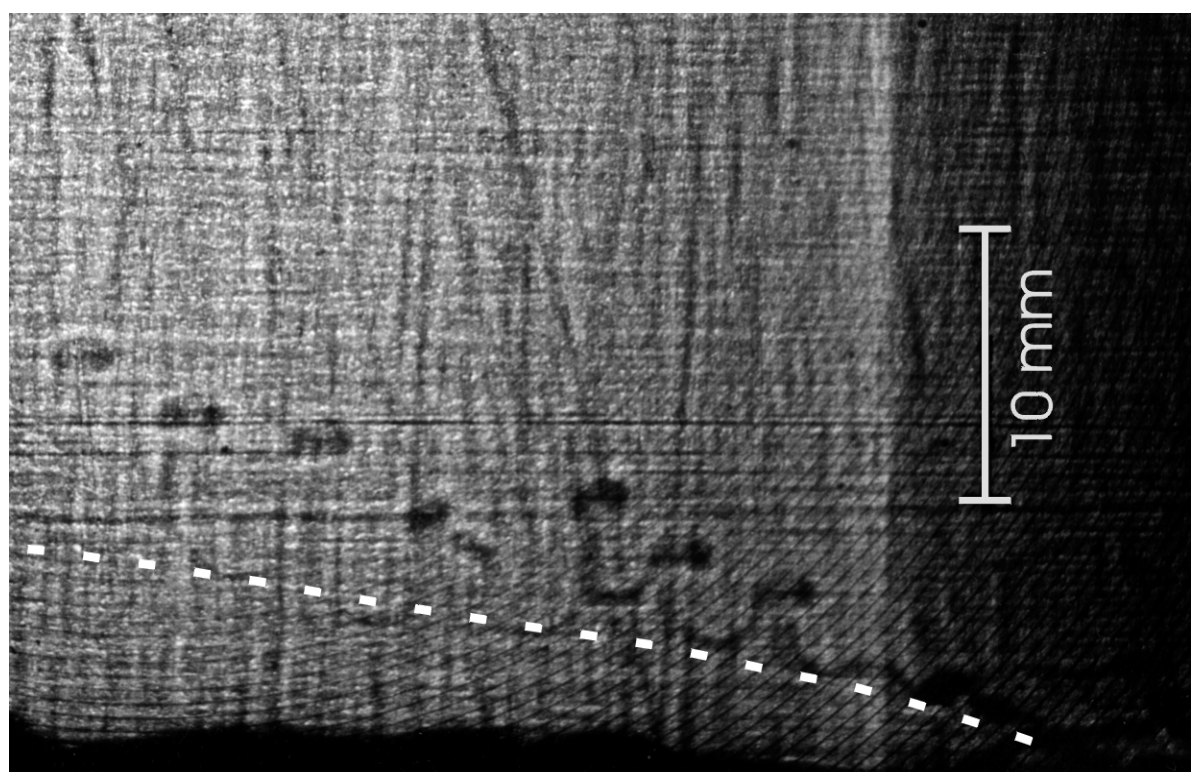

\section{Abbildung 8.15: Schattenbild eines gescherten Plumes}

Ein Plume wird plötzlich in Rotation versetzt und dadurch von seiner Umgebungsflüssigkeit geschert. Er wird in seiner gesamten Länge instabil und es lösen sich sekundäre Flüssigkeitstropfen ab. Gestrichelt eingezeichnet ist der aufgebrochene ursprüngliche Plumestamm, der an einigen Stellen noch zu erkennen ist.

er die kritische Höhe $H_{\text {Inst }}$ erreicht hat. Der Winkel nimmt dort nahezu $80^{\circ}-90^{\circ}$ mit der Horizontalen ein.

\subsubsection{Kleine Ekmanzahlen}

Der Einfluss von Rotation auf die Plumekonvektion wird in Abbildung 8.17] A-E demonstriert. Die Anfangskonzentration beträgt $C_{\circ}=26$ gew\%, die Unterkühlung $\Delta T=15 \mathrm{~K}$ und die Ekmanzahl variiert von $E=1,5 \cdot 10^{-4}$ bis $1,5 \cdot 10^{-5}$. Abbildung 8.17 A zeigt zum Vergleich nochmals die Plumekonvektion des nicht rotierenden Referenzexperiments. Die typische langgestreckte Helixstruktur des Plumeschlauchs ist deutlich zu erkennen. Die Strömungsstruktur der Fälle B-E unterscheiden sich hiervon jedoch deutlich.

In allen gezeigten Fällen kommt es zu dem oben beschriebenen Aufbrechen des Plumestamms in kleinere Flüssigkeitströpfchen. Mit zunehmender Winkelgeschwindigkeit $\Omega$ von (B nach E) wird der Plume in immer geringeren Höhen instabil. Die Höhendifferenz zwischen dem Austrittsort des Plumes und der Höhe, in der diese Instabilität auftritt, wird mit $H_{\text {Inst }}$ bezeichnet. In Abbildung 8.16 ist $H_{\text {Inst }}$ normiert auf die Zylin- 


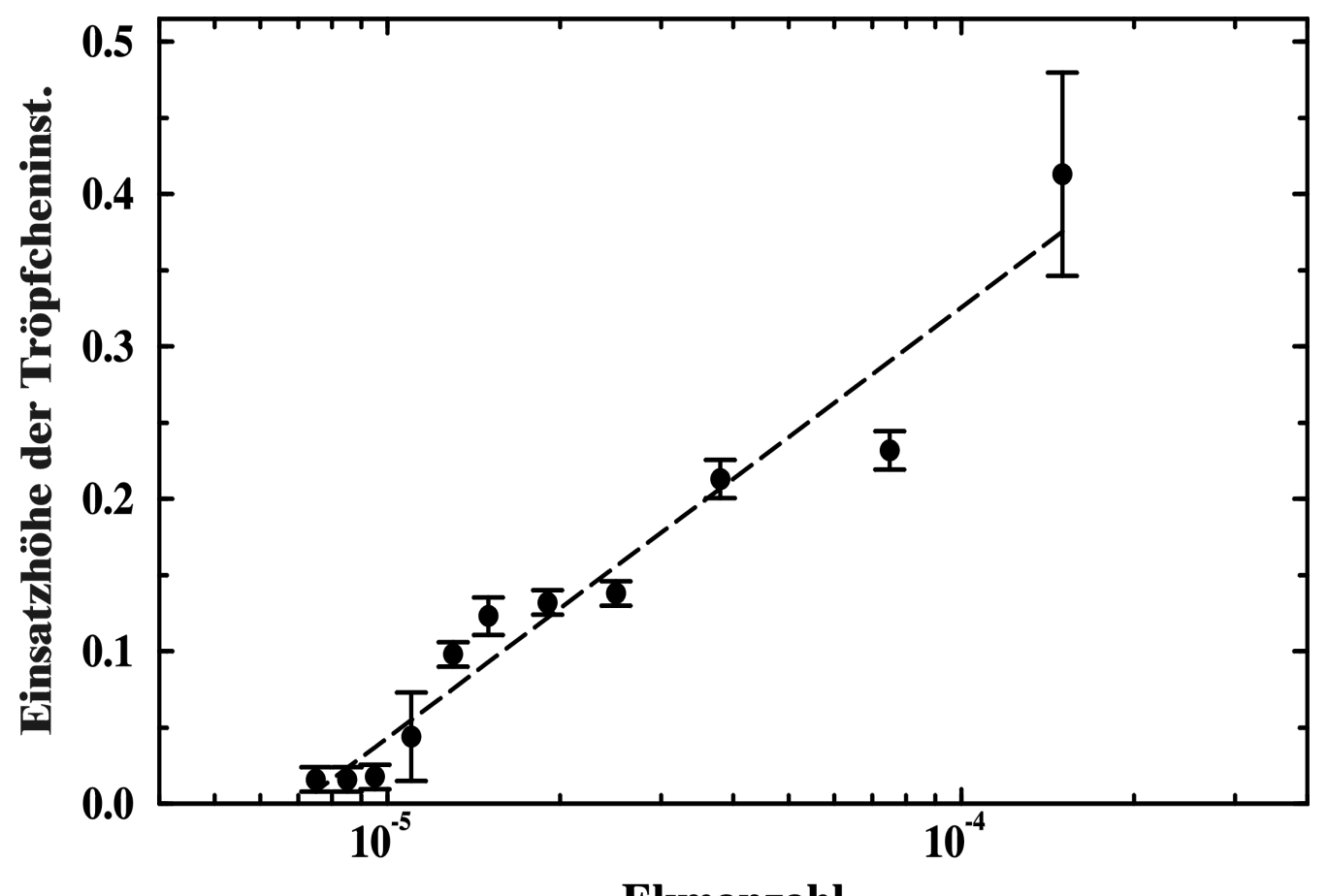

Ekmanzahl

Abbildung 8.16: Höhe, in der die Tröpfcheninstabilität einsetzt

Die Höhendifferenz zwischen dem Austrittsort des Plumes aus dem Kamin und der Höhe $H_{\text {Inst }}$, in der die erste Tröpfcheninstabilität auftritt, hier mit der Höhe des Zylinders $H_{Z}$ normiert dargestellt. Die Punkte sind aus mehreren Experimenten gebildete Mittelwerte. Fehlerbalken setzen sich aus den Standardabweichungen und der Messgenauigkeit zusammen.

derhöhe in Abhängigkeit zur Ekmanzahl dargestellt. Die Fehlerbalken setzen sich aus den Standardabweichungen der Mittelwertbildungen und den Ungenauigkeiten in der Höhenbestimmung der einzelnen Experimente zusammen. Zu $E=1,5 \cdot 10^{-4}$ wurde nur ein einzelnes Experiment durchgeführt, in dem $H_{\text {Inst }}$ der einzelnen Plumes stark schwankte (vgl. Abb. 8.17 B), so dass der Fehlerbalken für diese Rotationsrate vergleichsweise groß ist.

Mit kleiner werdender Ekmanzahl, also mit steigendem Einfluss der Corioliskraft, sinkt die Einsatzhöhe der Tröpfcheninstabilität näherungsweise logarithmisch. Bei einer Ekmanzahl von $E=9,5 \cdot 10^{-6}\left(\Omega=8,4 \frac{\mathrm{rad}}{\mathrm{s}}\right)$ beträgt die Höhe bereits nur noch etwa $0,3 \mathrm{~mm}$. Mit einer weiteren Zunahme von $\Omega$ auf $9,4 \frac{\mathrm{rad}}{\mathrm{s}}\left(E=8,5 \cdot 10^{-6}\right)$ wird der Plume, unmittelbar nachdem er aus dem Kamin tritt, instabil ( $H_{\text {Inst }} \approx 0-0,1 \mathrm{~mm}$ ). Bei diesen Rotationsraten ist die Strömungsstruktur bereits so kleinskalig, dass einzelne Tropfen nahe an $H_{\text {Inst }}$ nur noch schwer aufzulösen sind. Erst in etwas größeren Höhen sind sie wieder einzeln zu erkennen. Die Einsatzhöhe der Instabilität scheint hingegen unabhängig vom Auftriebsfluss des Plumes (Radius, Geschwindigkeit) zu sein. 

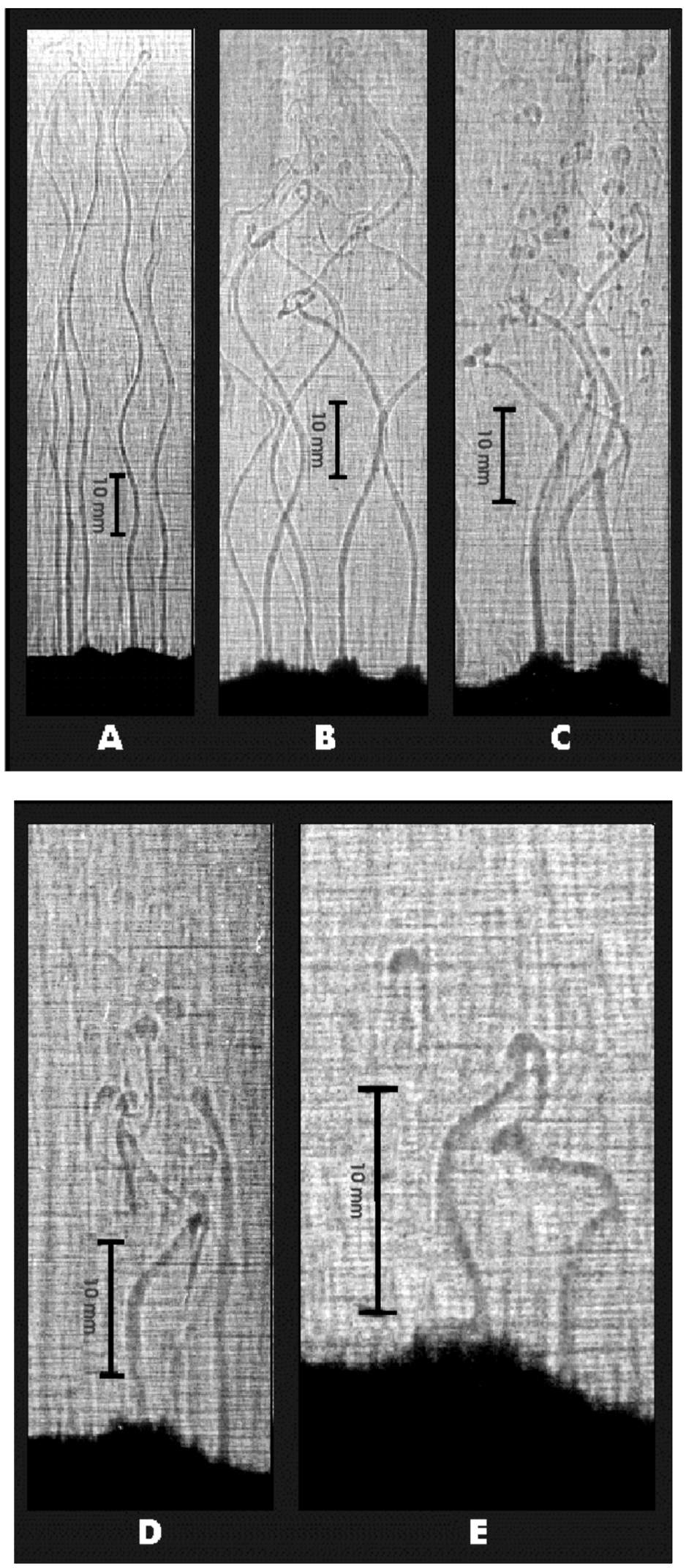

Abbildung 8.17: Schattenbilder von Plume- und Tröpfchenströmungsstrukturen bei unterschiedlichen Rotationsraten

$A: \Omega=0 \frac{\mathrm{rad}}{\mathrm{s}}, B: \Omega=0.5 \frac{\mathrm{rad}}{\mathrm{s}}\left(E=1.5 \cdot 10^{-4}\right), C: \Omega=1.1 \frac{\mathrm{rad}}{\mathrm{s}}\left(E=7.5 \cdot 10^{-5}\right), D: \Omega=3.1 \frac{\mathrm{rad}}{\mathrm{s}}$ $\left(E=2.5 \cdot 10^{-5}\right), E: \Omega=5.2 \frac{\mathrm{rad}}{\mathrm{s}}\left(E=1.5 \cdot 10^{-5}\right) ;$ alle Bilder: $C_{\circ}=26 \mathrm{gew} \%, \Delta T=15 \mathrm{~K}$, $\theta<2 K$. 


\section{Kapitel 9}

\section{Diskussion}

\section{Wechselwirkung zwischen Dendritenwachstum und Konvektion}

Stabilitätsanalysen der thermisch-chemischen Konvektion in porösen Schichten (in Analogie zu den Laborexperimenten), die auch nichtlineare Phänomene wie z. B. die Änderung der Schichtpermeabilität berücksichtigen (Anderson \& Worster, 1995 und Worster, 1997), finden folgende Stabilitätsbereiche (Bifurkationen) für die unterschiedlichen Strömungsgeometrien: Amberg \& Homsy (1993) finden stabile 2-D Rollenkonvektion und hexagonale Konvektion, bei der sich an den Rändern die Abströme und im Zentrum die Aufströme befinden. Anderson \& Worster (1995) dagegen beobachten, dass sich die inverse Form stabilisiert, mit Aufströmen an den Rändern und Abströmen in der Mitte. Eine wesentliche Beschränkung dieser Analysen ist jedoch, dass keine vertikalen Strömungen an der oberen Grenzfläche fest/flüssig zugelassen werden. Die Theorien sind auf das vorliegende Problem angepasst, aber stark vereinfacht.

In experimentellen Arbeiten von Tait et al. (1992) und Jaupart \& Tait (1995) werden hexagonale Strukturen in der porösen Dendritenschicht identifiziert. Sie verwenden eine 28 gew\%-ige $\mathrm{NH}_{4} \mathrm{Cl}-\mathrm{H}_{2} \mathrm{O}$ Lösung und kühlen die Lösung langsam ab. In der porösen Schicht bildet sich ein polygonales Muster von Kaminen und horizontalen, langgestreckten und hochporösen Verzweigungen. Die Verzweigungen füllen sich im Laufe der Zeit größtenteils mit Dendriten und in einigen Bereichen der Verzweigungen entstehen Kamine. Dieses Muster der Kamine und Wege in der Schicht wird statistisch nach der mittleren Anzahl der Verzweigungen, die von einem Kamin ausgehen, ausgewertet. Der so erhaltene mittlere Wert von 2,8 liegt nahe 3, dem Wert für ein ideales hexagonales Muster. Die Strömung in dieser hexagonalen Struktur wird von Aufströmen an den Rändern (durch die Kamine) und Abströmen im Mittelbereich (diffuse Rückströme) 
gebildet.

Ein ähnliches Muster kann in den hier durchgeführten Experimenten zur genaueren Untersuchung der Protokamine (Kap. 7.2) beobachtet werden. Die experimentellen Bedingungen sind gut vergleichbar mit den Versuchen von Tait et al. (1992), obwohl die Anfangskonzentration der Lösung etwas geringer ist und im Gegensatz zu Tait et al. (1992) gleichmäßig unterkühlt wird. Die Unterkühlung ist jedoch so gering, dass die Wachstumsraten beider Experimente vergleichbar sind.

Betrachtet man Abbildung 7.4 E und F, so findet man auch hier ein Muster von Kaminen und Verzweigungen in der Dendritenschicht. Verbindet man die Kamine entlang der Verzweigungen und deren Verlängerungen, so entsteht ein polygonales Netzwerk, in dem man drei-, vier-, fünf- oder sechseckige Strukturen finden kann, die vergleichbar mit den Mustern in den Experimenten von Tait et al. (1992), Bild 2 und 3, und Jaupart \& Tait (1995), Bild 10 sind. In den hier durchgeführten Versuchen konnte jedoch gezeigt werden, dass diese Muster durch die frühe Wachstumsgeschichte in der Dendritenschicht verursacht werden. Die Kamine und Wege (Verzweigungen) entstehen bevorzugt in den hochporösen Bereichen im Zentrum und in der Raumdiagonalen der kubischen Dendritenverbände. Dabei entsteht ein unregelmäßiges polygonales Muster und kein quadratisches, da die Keime statistisch entstehen und sich beim späteren Wachstum überschneiden. Auch bei vollentwickelter Plumekonvektion, wenn Verzweigungen bzw. Wege zum größten Teil mit Dendriten gefüllt sind, ordnen sich die Kamine nicht um. Ebensowenig konnte das Entstehen eines Kamins außerhalb der hochporösen Diagonalen beobachtet werden. Das Kaminmuster kann also nicht als das Resultat einer selbstorganisierten Konvektion angesehen werden. In Gebieten außerhalb der Protokamine dürfte die Entstehung neuer Kamine und die Bewegung vorhandener Kamine durch die geringe Permeabilität und damit große Reibung verhindert werden. Die Kristallisation als primärer Prozess bestimmt somit den weiteren Verlauf der Konvektion.

Bei den Experimenten hat sich gezeigt, dass die Unterkühlung einen großen Einfluss auf Kristallisation und Konvektion hat. Bei geringer Unterkühlung entstehen, in Übereinstimmung mit der klassischen Keimbildungstheorie, weniger, aber dafür größere Keime an homogen verteilten Positionen.

An diesen Keimen kristallisiert stark lokalisiert relativ viel Material, so dass viel niedrigkonzentrierte Lösung übrigbleibt. Der Konzentrationsgradient zu der darüberliegenden Flüssigkeit kann zu starken Aufströmen führen, so dass im Experiment (Proto-) Plumes beobachtet werden. Der Aufstrom zieht andererseits auch mehr konzentrierte Lösung von der Seite in den Dendritenverband hinein, Material, das dort kristallisieren 
kann. Durch diesen selbstverstärkenden Mechanismus wird das Wachstum des Dendriten gefördert und der Plume stabilisiert und fixiert. In den Experimenten entstand der Plume meistens im Zentrum des Dendritenverbandes, seltener in einer der porösen Diagonalen. Durch die Konvektion wird anscheinend begünstigt, dass im Zentrum des Dendriten ein offener Kanal entsteht. Die bisher in der Literatur beschriebenen Dendriten weisen dort dagegen einen Bereich größter Dichte auf. Der Grund für diesen erstaunlichen Unterschied könnte in der hier verwendeten geringen Unterkühlung zu finden sein.

Bei großer Unterkühlung entstehen viele kleine Kristallite, die schon im Anfangsstadium des Wachstums zu einer geschlossenen Schicht zusammenwachsen. Somit gibt es keine ausgezeichneten Positionen, an denen Plumes entstehen können, und damit wird die im Experiment beobachtete kleinskalige Fingerkonvektion bevorzugt.

Da die Kristallite die ideale ebene Bodenfläche zerstören, kann der Einfluss der Unterkühlung auf die Kristallitanzahl auch als von außen vorgegebene Variation der Störungswellenzahl betrachtet werden. Damit kann ein quantitativer Vergleich mit einer linearen Stabilitätsanalyse von Worster (1992) versucht werden. In Übereinstimmung mit den hier durchgeführten Experimenten wird bei geringer Störungswellenzahl (d. h. geringer Unterkühlung im Experiment) Plumekonvektion vorhergesagt und bei größerer Störungswellenzahl Fingerkonvektion. Es bleibt hier offen, inwieweit diese einfache lineare Stabilitätsanalyse qualitativ auf das verwendete komplexe Modellsystem anwendbar ist.

\section{Einfluss der Rotation auf das Kristallwachstum und den Einsatz der Plu- mekonvektion}

In den durchgeführten Experimenten konnte kein Einfluss der Rotation auf das Wachstum der Dendritenschicht beobachtet werden. So verändern sich in dem hier untersuchten Bereich der Winkelgeschwindigkeit weder die Permeabilität der Schicht, noch die Wachstumsgeschwindigkeit oder die Schichthöhe beim Einsatz der Konvektion. Die aus diesen Parametern berechnete kritische Rayleighzahl für den Konvektionseinsatz ist somit unabhängig von der Rotation. Dennoch verändert die Rotation die Form der Konvektion. So zeigt die Messung der Plumeanzahl $N_{P}$ zum Zeitpunkt des Konvektionseinsatzes, dass $N_{P}$ mit steigender Winkelgeschwindigkeit siginifikant abnimmt, ein Effekt, der bisher noch nicht beobachtet wurde. Darin liegt ein scheinbarer Widerspruch zu der Beobachtung, dass die Kristallisation von der Rotation unbeeinflusst bleibt und damit der gleiche Materialfluss stattfinden sollte. Um diese Diskrepanz zu klären, sei 
noch einmal kurz an das Taylor-Proudman Theorem (TPT) erinnert.

So besagt das TPT, dass in einem durch Corioliskräfte dominierten System, außerhalb von viskosen Grenzschichten, keine Variationen der Geschwindigkeit $\mathbf{u}=(u, v, w)$ in Richtung der Winkelgeschwindigkeit $\Omega$ auftreten dürfen. Bei Rotation um die vertikale Achse (z-Achse), wie in den hier durchgeführten Experimenten, gilt also: $\delta \mathbf{u} / \delta \mathbf{z}=0$. Liegt ein fester Rand senkrecht zu z vor (im Experiment der Deckel), verschwindet die Geschwindigkeitskomponente $w$ dort und laut TPT muß $w=0$ überall gelten. Die resultierende Strömung sollte demnach rein zwei-dimensional in einer Ebene sein. Bei Störungen entstehen parallel zur Rotationsachse sogenannte Taylorsäulen. Im vorliegenden Fall tritt jedoch Konvektion auf, die in vertikaler Richtung Wärme und Material transportieren muss und so das TPT verletzt. Die Corioliskraft kann den Einsatz der Konvektion hemmen oder sogar verhindern, setzt jedoch Konvektion ein, gilt das TPT nicht mehr oder nur noch beschränkt.

In dieser Arbeit wurde gezeigt, dass die Corioliskraft auf Grund der großen Reibung keinen Einfluss auf das Kristallwachstum und auf die Konvektion innerhalb der Dendritenschicht haben kann $\left(24 \leq E_{m} \leq 500\right)$. Demnach sollte auch die Bildung von Kaminen nicht behindert werden (im Experiment konnte dies auf Grund der hohen Rotationsrate nicht detailliert untersucht werden). Sobald jedoch der Aufstrom aus dem Kamin in die flüssige Phase aufsteigt, dominiert die Corioliskraft über die Reibung und der Aufstieg des Plumes wird unterdrückt. Nur einzelne Plumes mit großem Auftrieb können aufsteigen. Der Materialtransport wird von der gleichzeitig stattfindenden Fingerkonvektion übernommen. Wächst die Dendritenschicht und damit die Rayleighzahl $\left(R_{m} \propto H\right)$, wird eine immer größer werdende Anzahl $N_{P}$ beobachtet, bis sich das rotierende System nicht mehr von dem nicht-rotierenden unterscheidet.

Ein weiterer Einfluss der Rotation auf die Konvektion wird im Abschnitt Tröpfcheninstabilität behandelt.

\section{Plumeinstabilitäten}

Im Folgenden werden die unterschiedlichen Instabilitäten diskutiert, die bei der Plumekonvektion in rotierenden und nichtrotierenden Systemen auftreten.

\section{a. Helixstruktur $\left(\Omega=0 \frac{\mathrm{rad}}{\mathrm{s}}\right)$}

Aus Laborexperimenten mit wässrigen Ammoniumchloridlösungen (z. B. Chen \& Chen, 1991; Tait \& Jaupart, 1992; Jellinek et al., 1999) sind verschiedene Instabilitäten der Plumekonvektion bekannt. Die häufigste Instabilität, die in allen Experi- 
menten beobachtet wurde, ist die laterale Bewegung des Plumeschlauchs, die einen helikalen Weg induzieren können. In einigen Fällen, wie auch in dieser Arbeit, können auch laterale Verdickungen im Plumeschlauch (Abb. 8.5) entstehen. Eltayeb \& Loper (1997) konnten zeigen, dass die helikale Instabilität das Resultat der viskosen Reibung zwischen ausfteigendem Plume und absteigender Umgebungsflüssigkeit ist. Dominiert die thermische Diffusivität über die viskosen Kräfte der Strömung (große Prandtlzahl), so ist die Instabilität achsensymmetrisch. Es entstehen Verdickungen im Plume, wodurch die Oberfläche vergrößert und der Wärmeaustausch mit der Umgebung erhöht wird. Damit werden die thermischen Einflüsse, die die Strömung behindern, verringert. Bei kleinen Prandtlzahlen dominieren die viskosen Kräfte über die thermischen Einflüssen. Für diesen Fall zeigt die Stabilitätsanalyse von Eltayeb \& Loper, dass die Instabilität einen helikalen Charakter hat. So wird die viskose Reibung verringert, da die entgegenfließende Flüssigkeit auf eine Seite des Plumes „geschoben“ wird.

Im Experiment ist die Prandtlzahl $(\operatorname{Pr}=9)$ zwar noch nicht in dem Bereich, in dem die viskosen Kräfte größer als die thermischen Einflüsse sind; die viskosen Kräfte sind anscheinend dennoch bereits groß genug, die von Eltayeb \& Loper beschriebene helikale Instabilität zu verursachen.

\section{b. Tröpfcheninstabilität $\left(\Omega \neq 0 \frac{\mathrm{rad}}{\mathrm{s}}\right)$}

In den wenigen Kristallisationsexperimenten mit Ammoniumchlorid oder ähnlichen Systemen, in denen der Einfluss der Rotation untersucht wurde (Sample \& Hellawell, 1982, 1984 und Neilson \& Incropera, 1993b), lag der Schwerpunkt der Untersuchungen in der Kristallisation und der Entstehung der Kamine und weniger in der Konvektion. Im Bereich der Konvektion deuten die zwei-dimensionalen numerischen Simulationen von Neilson \& Incropera (1993a) darauf hin, dass die Coriolisbeschleunigung die Plumekonvektion stabilisieren sollte. Der Plume verliert demnach seine helikale Instabilität und steigt geradlinig vertikal auf. Das in dieser Arbeit beobachtete Auftreten einer Instabilitätsverstärkung bei der Plumekonvektion unter dem Einfluss von Rotation ist daher zunächst überraschend.

Eltayeb \& Hamza (1998) untersuchen die Stabilität eines Plumes, der durch Konzentrationsdifferenzen angetrieben wird und in einem rotierenden System aufsteigt. Sie finden, dass die Störungen, die ein Plume durch viskose Reibung erfährt, unter dem Einfluss der Corioliskraft verstärkt werden. Die Ausbildung einer helikalen Instabilität führt zu einer transversalen Geschwindigkeitskomponente v, die mit der in vertikaler Richtung weisenden Winkelgeschwindigkeit $\Omega$ interagiert und so zu einer Corioliskraft 
$\mathbf{F}_{\mathbf{C}}$ führt $\left(\mathbf{F}_{\mathbf{C}}=2 m \boldsymbol{\Omega} \times \mathbf{v}\right)$. Störungen im Plume können durch diesen Effekt verstärkt werden und so zu Instabilitäten führen, die ein Aufbrechen des Plumes verursachen. Nach Eltayeb \& Hamza (1998) hängt die Wachstumsrate dieser Instabilitäten von der Taylorzahl ab $\left(T a=\left(\frac{1}{E}\right)^{2}\right)$. Für $T a \leq 1$ steigt die Wachstumsrate zunächst steil an und geht dann ab $T a \approx 4$ in ein asymptotisches Verhalten über. Als Längenskala $L$ legen Eltayeb \& Hamza (1998) die Länge der Fingerkonvektion zu Grunde. In den hier durchgeführten Experimenten mit einem typischen Fingerabstand von $L=0,5 \mathrm{~mm}$ liegt die Taylorzahl somit in dem Bereich $0,05 \leq T a \leq 19$. Nimmt man an, dass der Plume unmittelbar nach Austritt aus dem Kamin Instabilitäten ausgesetzt ist, so kann man die Höhe bis zu seinem Aufbrechen mit der Wachstumsrate der Instabilität korrelieren.

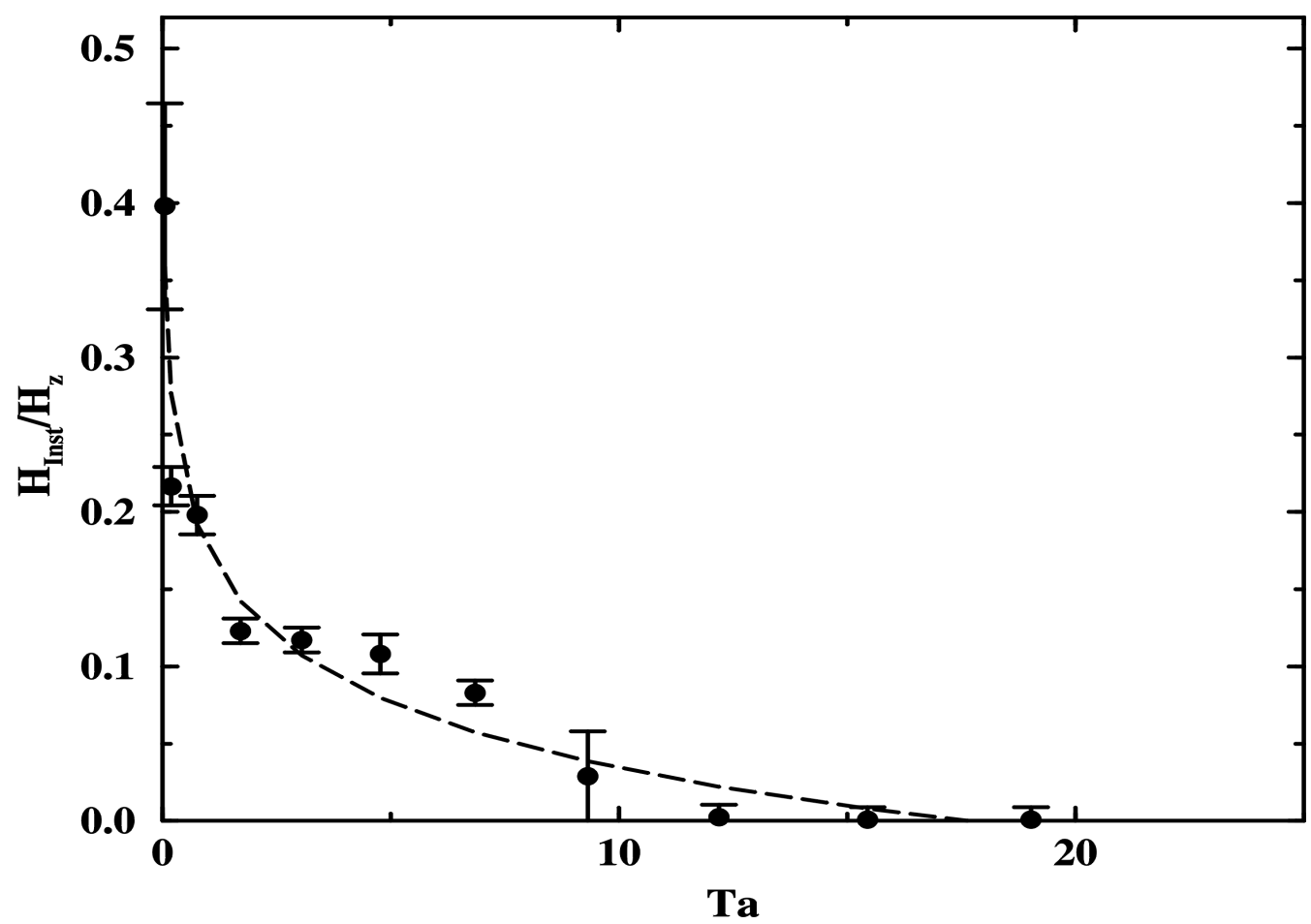

\section{Abbildung 9.1: Höhe, in der die Tröpfcheninstabilität einsetzt}

Die normierte Höhe $H_{\text {Inst }}$ der Tröpfcheninstabilität nimmt schnell mit steigender Taylorzahl Ta ab. Ab Ta $\approx 12$ setzt die Instabilität unmittelbar an der Wachstumsfront ein. ( $H_{Z}=$ Höhe des Kontainers).

In Abbildung 9.1 wird die Höhe $H_{\text {Inst }}$, in der die Tröpfcheninstabilität einsetzt, gegen die Taylorzahl aufgetragen. $H_{\text {Inst }}$ sinkt sehr schnell mit $T a$ bis zu $T a \approx 1$ und nähert sich dann langsam asymptotisch einer Höhe von Null. Dort setzt die Instabilität direkt an der Oberfläche der Dendritenschicht ein. Die Einsatzhöhe der Instabilität macht ei- 
ne Aussage darüber, wie lange ein Plume aufsteigen kann, bevor eine kleine Störung so weit anwachsen konnte, dass der Plume instabil wird und aufbricht. Die Wachstumsgeschwindigkeit der Störung ist also direkt mit $H_{\text {Inst }}$ verknüpft, das heißt mit steigender Rotation (Taylorzahl) wird der Plume schneller (in geringerer Höhe) instabil und bricht in kleine Tröpfchen auf.

\section{Anwendung auf das System Erde}

Bevor man die Relevanz der Ergebnisse des verwendenten Modellsystems für die Prozesse im Erdkern diskutiert, müssen die Unterschiede zwischen den Laborexperimenten und der Erde betrachtet werden. Zum einen bedingt die Geometrie in den Versuchen $(\Omega \| \mathbf{g})$, dass der Einfluss der Corioliskraft nur für die Polregionen des Kerns modelliert werden kann. Jedoch liegt die Ekmanzahl im Erdkern mit 10 $10^{-10}-10^{-15}$ (Melchior, 1986) weit unterhalb der Werte, die im Labor zugänglich sind $\left(E=1,5 \cdot 10^{-4}-7,5 \cdot 10^{-6}\right)$, und auch die modifizierte Reynoldszahl $R e=\frac{\Omega \cdot R^{2}}{\nu}\left(\mathrm{mit}: \Omega=7,29 \cdot 10^{-5} \frac{\mathrm{rad}}{\mathrm{s}}, R=2258 \mathrm{~km}\right.$ und $\nu \approx 1 \cdot 10^{-6} \frac{\mathrm{m}^{2}}{\mathrm{~s}}$ (Melchior, 1986)) liegt mit etwa $4 \cdot 10^{14}$ weit oberhalb der Werte, die im Experiment realisiert werden können $\left(R e=6,7 \cdot 10^{2}-1,4 \cdot 10^{4}\right)$. Die Extrapolation der Ergebnisse auf den Erdkern muss darum spekulativ bleiben.

Die Experimente zeigen, dass die Tendenz zur Bildung der Tröpfcheninstabilität mit sinkender Ekmanzahl stark zunimmt. In der Erde ist daher zu erwarten, dass die Plumekonvektion keine entscheidende Rolle spielt. Plumes sollten direkt nach Verlassen der ICB in Tröpfchen aufbrechen.

Für die Konvektion im äußeren Erdkern spielt die Lorentzkraft eine wichtige Rolle. Man weiss, dass starke Magnetfelder Störungen bzw. Instabilitäten dämpfen oder verhindern können (Chandasekhar, 1981). Dieser Einfluss kann in dem hier betrachteten Modellsystem nicht untersucht werden, da die verwendete Flüssigkeit nur eine geringe elektrische Leitfähigkeit besitzt. Hierzu geeignete metallische Legierungen wie $\mathrm{Pb}-\mathrm{Sn}$ sind jedoch nicht transparent, so dass Strömungsinstabilitäten nicht visualisiert werden können. Eltayeb (1999) zeigte jedoch in Stabilitätsanalysen eines zylindersymmetrischen Aufstroms (entsprechend zu den hier vorliegenden Plumes) in einem rotierenden System mit Magnetfeld, dass ein Plume für alle Werte der Taylorzahl und Chandasekharzahl instabil wird.

Die Größe der in diesen Experimenten beobachteten Tröpfchen liegt in der gleichen Größenordnung wie der Kamindurchmesser. Auf die Erde übertragen sollte die Strömungsstruktur nur wenige $100 \mathrm{~m}$ betragen. Damit ist sie viel zu kleinskalig, als dass sie an der Erdoberfläche nachgewiesen werden könnte. Zur Erzeugung eines Dyna- 
mos werden jedoch großskalige Strömungen benötigt. Shimizu \& Loper (1997) konnten zeigen, dass eine kleinskalige, stark lokalisierte Strömungsstruktur unter erdähnlichen Bedingungen zu einer großskaligen Strömung, ähnlich den Taylorsäulen, führen kann. Diese könnten wiederum die für den Erddynamo entscheidenden $\alpha$-oder $\omega$ - Effekte verursachen.

In dieser Arbeit wurde kein signifikanter Einfluss der Corioliskraft auf die Morphologie der Dendritenschicht beobachtet. Es sei jedoch daran erinnert, dass die Wachstumsrichtung der Dendriten vor allem durch die Richtung des Wärmeflusses bestimmt wird. Da hier $\Omega$ und $\mathbf{g}$ parallel sind, ist damit keine Abhängigkeit von $\Omega$ zu erwarten. Im Fall des Erdkerns kann jedoch der Wärmefluss durch die Stömungsstrukturen im äußeren Kern modifiziert werden. So schlagen Yoshida et al. (1996) vor, dass es durch den verstärkten Wärmefluss in äquatorialer Richtung zu einer Ausrichtung der Dendriten kommen sollte. Nach Bergmann (1997) könnte diese Textur die elastische Anisotropie des inneren Kerns erklären.

Experimente von Tewari et al. (1994) mit einer Pb-Sn Legierung darauf hin, dass das experimentell zugängliche Magnetfeldes $(0,45 T)$ die Morphologie einer porösen Dendritenschicht und die Entstehung von Kaminen nicht signifikant beeinflusst. Jedoch konnte auch in diesen Experimenten keine Chandrasekharzahl größer als eins realisiert werden, die benötigt wird, damit, wie in der Erde, die magnetischen Kräfte die viskosen übertreffen (Bergman \& Fearn, 1994). Somit bleibt offen, ob das Magnetfeld eine Texturierung verursachen könnte. 


\section{Kapitel 10}

\section{Zusammenfassung}

In dieser Arbeit wird systematisch der Einfluss von Corioliskräften auf das Kristallwachstum und die Konvektion in einem binären eutektischen Modellsystem im Hinblick auf die Vorgänge an der Grenze zwischen innerem und äußerem Erdkern untersucht.

In den Experimenten kann keine Beeinflussung der Kristallistation durch die Rotation festgestellt werden, weder Wachstumsgeschwindigkeit noch Morphologie der porösen Dendritenschicht werden verändert. Entscheidender für die Kristallisation ist die Unterkühlung. So entstehen bei geringer Unterkühlung (langsames Wachstum) im Frühstadium der Kristallisation kleine (Proto-) Kamine in der Mitte der Dendritenverbände, aus denen konzentrierte Aufströme (Protoplumes) aufsteigen. Es konnte nachgewiesen werden, dass diese Protokamine die Lage der vollentwickelten Kamine festlegen und damit die Geometrie der Strömung bestimmen.

In Experimenten mit hoher Unterkühlung und damit gleichmäßiger, schneller Kristallisation entstehen keine Protokamine und es findet keine Plumekonvektion im Frühstadium des Wachstums statt.

Bei Rotation spielt die Corioliskraft gegenüber der Darcy-Reibung in einem porösen Medium eine untergeordnete Rolle, so dass die Konvektion in der porösen Dendritenschicht und damit die Kaminbildung nicht behindert wird. Der Einsatz der Plumekonvektion findet wie im nicht-rotierenden Fall bei $R_{m_{c}} \approx 200$ statt. Mit zunehmender Rotation (sinkender Ekmanzahl) nimmt die Plumeanzahl beim Konvektionseinsatz stark ab. Die Plumekonvektion wird also mit anwachsender Corioliskraft unterdrückt.

Einen weiteren entscheidenden Einfluss der Rotation erfährt die Strömungsstruktur in der flüssigen Phase oberhalb der Dendritenschicht. Bei Rotation wird ein Plume an seinem vertikalen Aufstieg gehindert, der Steigungswinkel der Helix sinkt unter einen 
kritischen Winkel und der Plumestamm bricht in eine Vielzahl kleiner unabhängiger Tröpfchen auf. Diese Instabilität wird mit steigender Rotation verstärkt. Ab $E \approx$ $9,5 \cdot 10^{-6}$ kann sich kein zusammenhängender Plumeschlauch mehr bilden, da er sofort nach Verlassen der Dendritenschicht aufbricht.

Im flüssigen äußeren Erdkern $\left(E \approx 10^{-15}\right)$ sollten daher Plumes, die aus der porösen Grenzschicht (ICB) zwischen innerem und äußerem Kern aufsteigen, in Tröpfchen aufbrechen. Diese Tröpfcheninstabilität könnte demnach zu einer kleinskaligen Konvektion mit einer Längenskala von wenigen hundert Metern führen. Ihre Relevanz für den Erdkern und den Erddynamo zu klären, steht jedoch noch aus. 


\section{Literaturverzeichnis}

[1] G. Amberg und G. M. Homsy. Nonlinear analysis of buoyant convection in binary solidification with application to channel formation. Journal of Fluid Mechanics, 252:79-98, 1993.

[2] D. M. Anderson und M. G. Worster. Weakley nonlinear analysis of convection in mush layers during the solidification of binary alloys. Journal of Fluid Mechanics, 302:307-331, 1995.

[3] D. R. Askeland. Materialwissenschaften. Spektrum Akademischer Verlag, Heidelberg, 1996.

[4] G. K. Batchelor. An Introduction to Fluid Dynamics. Cambridge University Press, Cambridge, 1994.

[5] J. Bear. Dynamics of fluids in porous media. Dover Publications, Inc., New York, 1972.

[6] M. I. Bergman. Measurements of elastic anisotropy due to solidification texturing and the implications for the Earth's inner core. Nature, 389:60-63, 1997.

[7] M. I. Bergman. Estimates of the Earth's inner core grain size. Geophysical Research Letters, 25-10:1593-1596, 1998.

[8] M. I. Bergman. A laboratory model for the solidification of the Earth's inner core, and the inner core's seismic anisotropy. IUGG 99, Abstracts A, JSS07/W/03A2:A.43, 1999.

[9] M. I. Bergman, D. R. Fearn, J. Bloxham und M. C. Shannon. Convection and channel formation in solidifying Pb-Sn alloys. Metall. Trans., 28A:859-866, 1997.

[10] M. I. Bergman und D. R. Fearn. Chimneys on the Earth's inner-outer core boundary? Geophysical Research Letters, 21:477-480, 1994. 
[11] J. Bhattacharyya, P. Shearer und G. Masters. Inner core attenuation from shortperiod PKP(BC) versus PKP(DF) waveforms. Geophysical Journal International, 114:1-11, 1993.

[12] B. A. Buffett, H. E. Huppert, J. R. Lister und A. W. Woods. Analytical model for solidification of the Earth's core. Nature, 356:329-331, 1992.

[13] B. A. Buffett, H. E. Huppert, J. R. Lister und A. W. Woods. On the thermal evolution of the Earth's core. Journal of Geophysical Research, 101:7989-8006, 1996.

[14] F.H. Busse. Thermal instabilities in rapidly rotating systems. Journal of Fluid Mechanics, 44:441-460, 1970.

[15] F.H. Busse. Convection driven zonal flows and vortices in the major planets. CHAOS, 4:123-134, 1994.

[16] P. Cardin und P. Olson. An experimental approach to thermochemical convection in the Earth's core. Geophysical Research Letters, 19:1995-1998, 1992.

[17] C. R. Carrigan und F.H. Busse. An experimental anf theoretical investigation of the onset of convection in rotating spherical shells. Journal of Fluid Mechanics, 126:287-305, 1983.

[18] S. Chandrasekhar. Hydrodynamic and Hydromagnetic Stability. Dover Publications, Inc., New York, 1961.

[19] F. Chen, J. W. Lu und T. L. Yang. Convective instabilities in ammonium chloride solution directionally solidified from below. Journal of Fluid Mechanics, 276:163$187,1994$.

[20] F. Chen, T. L. Yang und J. W. Lu. Influence of convection on solidification of binary solutions cooling from below. J. Appl. Phys., 74:7531-7541, 1993.

[21] C. F. Chen und F. Chen. Experimental study of directional solidification of aqueous ammonium chloride solution. Journal of Fluid Mechanics, 227:567-586, 1991.

[22] G. L. Choy und V. F. Cormier. The structure of the inner core inferred from short-period and broadband GDSN data. Journal of Geophysical Research, 72:121, 1983. 
[23] S. M. Copley, A. F. Giamei, S. M. Johnson und M. F. Hornbecker. The origin of freckles in binary alloys. J. Appl. Math., 35:159-174, 1970.

[24] V. F. Cormier und P. G. Richards. Comments on 'The damping of core waves' by A. Qamar und A. Eisenberg. Journal of Geophysical Research, 81:3066-3068, 1976.

[25] K. C. Creager. Anisotropy of the inner core from differential travel times of the phases PKP and PKIKP. Nature, 356:309-314, 1992.

[26] P. Cummins und L. R. Johnson. Short-period body wave constraints of properties of the Earth's inner core boundary. Journal of Geophysical Research, 93:9058-9074, 1988a.

[27] P. Cummins und L. R. Johnson. Synthetic seismograms for an inner core transition of finite thickness. Journal of Geophysical Research, 94:21-34, 1988b.

[28] D. J. Doornbos. The anelasticity of the inner core. Journal of Geophysical Research, 38:397-415, 1974.

[29] I. A. Eltayeb. The stability of compositional plumes in a rotating magnetic fluid. Physics of the Earth and Planetary Interiors, 110:1-19, 1999.

[30] I. A. Eltayeb und D. E. Loper. On the stability of vertical double-diffusive interfaces. Part 3. Cylindrical interface. Journal of Fluid Mechanics, 353:45-66, 1997.

[31] I. A. Eltayeb und E. A. Hamza. Compositional convection in the presence of rotation. Journal of Fluid Mechanics, 354:277-299, 1998.

[32] P. W. Emms und A. C. Fowler. Compositional convection in the solidification of binary alloys. Journal of Fluid Mechanics, 262:111-139, 1994.

[33] K. H. Esbensen und V. F. Buchwald. Planet(oid) core crystallisation and fractionevidence from the Agpalilik mass of the Cape York iron meteorite shower. Physics of the Earth and Planetary Interiors, 29:218-232, 1982.

[34] D. R. Fearn, D.E. Loper und P. H. Roberts. Structure of the Earth's inner core. Nature, 292:232-233, 1981.

[35] P. Haasen. Physikalische Metallkunde. Springer Verlag, Berlin, Heidelberg, New York, 1994. 
[36] A. Hellawell, J. R. Sarazin und R. S. Steube. Channel convection in partly solidified systems. Phil. Trans. R. Soc. Lond. A, 345:507-544, 1993.

[37] H. E. Huppert. The fluid mechanics of solidification. Journal of Fluid Mechanics, 212:209-240, 1990.

[38] H. E. Huppert und M. G. Worster. Dynamic solidification of a binary melt. Nature, 314:703-707, 1985.

[39] K. A. Jackson, J. D. Hunt, D. R. Uhlmann und J. P. III Seward. On the origin of the equiaxed zone in castings. Trans. Metall. Soc., 236:149-158, 1966.

[40] C. Jaupart und St. Tait. Dynamics of differentiation in magma reservoirs. Journal of Geophysical Research, 100:17615-17636, 1995.

[41] R. Jeanloz und H. R. Wenk. Convection and anisotropy of the inner core. Geophysical Research Letters, 15:72-75, 1988.

[42] A. M. Jellinek, R. C. Ross und R. W. Griffiths. Mixing and compositional stratification produced by natural convection; 1 . Experiments and their application to Earth's core and mantle. Journal of Geophysical Research, 104:7183-7201, 1999.

[43] S. Karato. Inner core anisotropy due to magnetic field-induced preferred orientation of iron. Science, 262:1708-1711, 1993.

[44] W. Kleber. Einführung in die Kristallographie. VEB Verlag Technik, Berlin, 1983.

[45] W. Kurz und D. J. Fisher. Fundamentals of solidification. Trans Tech Publications Ltd, Aedermannsdorf (Switzerland), 1989.

[46] S. Labrosse, J.-P. Poirier und J.-L. Le Mouël. On cooling of the Earth's core. Physics of the Earth and Planetary Interiors, 99:1-17, 1997.

[47] J. R. Lister und B. A. Buffett. The strength and efficiency of thermal and compositional convection in the geodynamo. Physics of the Earth and Planetary Interiors, 91:17-30, 1995 .

[48] D. E. Loper. Structure of the inner core boundary. Geophys. Astrophys. Fluid Dynamics, 25:139-155, 1983.

[49] D. E. Loper und D. R. Fearn. A seismic model of a partially molten inner core. Journal of Geophysical Research, 88:1235-1242, 1983. 
[50] D. E. Loper und P. H. Roberts. A study of conditions at the inner core boundary of the Earth. Physics of the Earth and Planetary Interiors, 24:302-307, 1981.

[51] T. G. Masters und P. M. Shearer. Summary of seismological constraints on the structure of the Earth's core. Journal of Geophysical Research, 95:21691-21695, 1990.

[52] J. R. McDonald und J. D. Hunt. Convective fluid motion within the interdendritic liquid of a casting. Metall. Trans., 1:1787-1788, 1970.

[53] R. J. McDonald und J. D. Hunt. Fluid motion through the partially solid region of a casting and its importance in understanding a type segregation. Trans. Metall. Soc., 245:1993-1997, 1969.

[54] P. Melchior. The physics of the Earth's core. Pergamon Press, Oxford, 1986.

[55] H. K. Moffatt und D. E. Loper. The magnetostrophic rise of a buoyant parcel in the Earth's core. Geophysical Journal International, 117:394-402, 1994.

[56] A. D. Morelli, A. M. Dziewonski und J. H. Woodhouse. Anisotropy of the inner core inferred from PKIKP travel times. Geophysical Research Letters, 13:15451548, 1986.

[57] H. Müller-Krumbhaar und W. Kurz. Solidification. In P. Haasen und E. J. Kramer R. W. Cahn, editor, Materials science and tecnology, Vol. 5: Phase transformations in materials, pages 553-632. VCH Verlagsgesellschaft, Weinheim, New York, Basel, Cambridge, 1991.

[58] D. G. Neilson und F. P. Incropera. Effect of rotation on fluid motion and channel formation during unidirectional solidification of a binary alloy. Heat Mass Trans., 36:489-505, 1993a.

[59] D. G. Neilson und F. P. Incropera. Experimental study of unidirectional solidification of aqueous ammonium chloride in a cylindrical mold with and without rotation. Exp. Heat Transfer, 6:131-155, 1993b.

[60] H. Oertel sen. und H. Oertel jun. Strömungsmeßtechnik. G. Braun, Karlsruhe, 1989.

[61] G. Poupinet, R. Pillet und A. Souriau. Possible heterogeneity of the Earth's core induced from PKIKP travel times. Nature, 305:204-206, 1983. 
[62] B. Romanowicz, X. D. Li und J. Durek. Anisotropy in the inner core: Could it be due to low order convection? Science, 274:963-966, 1996.

[63] A. K. Sample und A. Hellawell. The effect of mold precession on channels and macro-segregation in ammonium chloride-water analog castings. Metall. Trans. B, 13B:495-501, 1982.

[64] A. K. Sample und A. Hellawell. The mechanisms of formation and prediction of channel segretation during alloy solidification. Metall. Trans. A, 15A:2163-2173, 1984.

[65] J. R. Sarazin und A. Hellawell. Channel formation in $\mathrm{Pb}-\mathrm{Sn}$, and $\mathrm{Pb}-\mathrm{Sn}-\mathrm{Sb}$ alloy ingots and comparison with the system $\mathrm{NH}_{4} \mathrm{Cl}-\mathrm{H}_{2} \mathrm{O}$. Metall. Trans. A, 19A:18611871, 1988.

[66] S. K. Saxena, L. S. Dubrovinsky, P. Haggkrist, Y. Cerenius, G. Shen, und H. K. Mao . Synchrotron X-ray study of iron at high pressure and temperature. Science, 269:1703-1704, 1995.

[67] P. M. Shearer und G. Masters. The density and shear velocity contrast at the inner core boundary. Geophysical Journal International, 102:491-498, 1990.

[68] H. Shimizu und D. E. Loper. Time and length scale of buoyancy-driven flow structures in a rotating hydromagnetic fluid. Physics of the Earth and Planetary Interiors, 104:307-329, 1997.

[69] T. G. L. Shirtcliffe, H. E. Huppert und M. G. Worster. Measurement of the solid fraction in the crystallization of a binary melt. Journal of Crystal Growth, 113:566-574, 1991.

[70] T. G. L. Shirtcliffe und R. C. Kerr. On the use of electrical resistance and temperatur as measures of the solid fraction of a mushy layer. Journal of Crystal Growth, 125:495-501, 1992.

[71] X. Song. Anisotropy in central part of inner core. Journal of Geophysical Research, 101:16089-16097, 1996.

[72] X. Song und D. V. Helmberger. Velocity structure near the inner core boundary from waveform modelling. Journal of Geophysical Research, 97:6573-6586, 1992.

[73] D. J. Stevenson. Inner core anisotropy (abstract). Eos Trans. AGU, Fall Meet. Suppl., 77(46):F41, 1996. 
[74] L. Stixrude und R. E. Cohen. High-pressure elasticity of iron anisotropy of Earth's inner core. Science, 267:1972-1975, 1995.

[75] W. Su und A. M. Dziewonski. Inner core anisotropy in three dimensions. Journal of Geophysical Research, 100:9831-9852, 1995.

[76] I. Sumita, S. Yoshida, M. Kumazawa und Y. Hamano. A model for sedimentary compaction of a viscous medium and its application to inner-outer core growth. Geophysical Journal International, 124:502-524, 1996.

[77] St. Tait, K. Jahrling und C. Jaupart. The planform of compositional convection and chimney formation in a mushy layer. nature, 359:406-408, 1992.

[78] St. Tait und C. Jaupart. Compositional convection in viscous melt. Nature, 338:571-574, 1989.

[79] St. Tait und C. Jaupart. Compositional convection in a reactive crystalline mush and melt differentiation. Journal of Geophysical Research, 97:6735-6756, 1992.

[80] S. N. Tewari, R. Shah und H. Song. Effect of magnetic field on the microstructure and macrosegregation in directionally solidified $\mathrm{Pb}-\mathrm{Sn}$ alloys. Metallurgical and Materials Transactions A, 25A:1535-1544, 1994.

[81] S. N. Tewari und R. Shah. Macrosegregation during steadt-state arrayed growth of dendrites in directionally solidified Pb-Sn alloys. Metallurgical Transactions A, 23A:3383-3392, 1992.

[82] S. N. Tewari und R. Shah. Macrosegregation during dendritic arrayed growth of hypoeutectic $\mathrm{Pb}-\mathrm{Sn}$ alloys: Influence of primary arm spacing and mushy zone length. Metallurgical and Materials Transactions A, 27A:1353-1362, 1996.

[83] D. J. Tritton. Physical Fluid Dynamics. Oxford Science Publications, Oxford, New York, 1988.

[84] J. Tromp. Support for anisotropy of the Earth's inner core from free oscillations. Nature, 366:678-681, 1993.

[85] J. S. Turner. Buoyancy in fluids. Cambridge University Press, Cambridge, 1973.

[86] J. A. Whitehead. Instabilities of fluid conduits in a flowing Earth-Are plates lubricated by the asthenosphere? Geophysical Research Letters, 70:415-433, 1982. 
[87] J. H. Woodhouse, D. Giardini und X. D. Li. Evidence for inner core anisotropy from free oscillations. Geophysical Research Letters, 13:1549-1552, 1986.

[88] M. G. Worster. Solidification of an alloy from a cooled boundary. Journal of Fluid Mechanics, 167:481-501, 1986.

[89] M. G. Worster. Natural convection in a mushy layer. Journal of Fluid Mechanics, 224:335-359, 1991.

[90] M. G. Worster. Instabilities of the liquid and mushy regions during solidification of alloys. Journal of Fluid Mechanics, 237:649-669, 1992.

[91] M. G. Worster. Convection in mushy layers. Ann. Rev. Fluid Mech., 29:91-122, 1997.

[92] S. Yoshida, I. Sumita und M. Kumazawa. Growth model of the inner core coupled with the outer core dynamics and the resulting elastic anisotropy. Journal of Geophysical Research, 101:28085-28103, 1996. 


\section{Abbildungsverzeichnis}

I. Innerer Aufbau der Erde . . . . . . . . . . . . . . . . . 7

3.1 Gerichtetes Wachstum eines eutektischen Systems . . . . . . . . . . . . 18

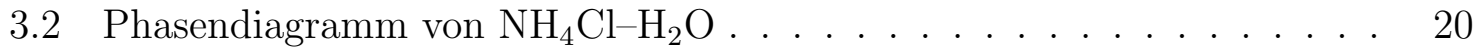

3.3 Planares und Dendritisches Kristall-Wachstum . . . . . . . . . . . . 23

B.4 Nahautnahme von Dendriten . . . . . . . . . . . . . . . . . . . 24

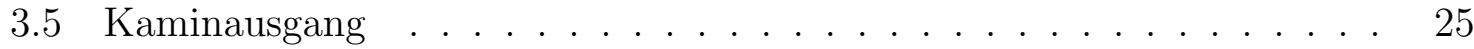

36 Dendritenschicht mit Plumes . . . . . . . . . . . . . . . 26

4.1 Versuchsaufbaul . . . . . . . . . . . . . . . . . . . . 30

4.2 Kühlbäder . . . . . . . . . . . . . . . . . . . . . . . . . . 31

4.3 Optischer Autbau $\ldots \ldots \ldots . \ldots . \ldots \ldots$

5.1 Bodentemperatur . . . . . . . . . . . . . . . . . . 37

5.2 Regulierung der äußeren Wasserbadtemperatur . . . . . . . . . . . . . 38

6.1 Konzentrationsprofil . . . . . . . . . . . . . . . . . . . . . . . 41

6.2 Kalibrierungskurve $\ldots \ldots \ldots \ldots \ldots$

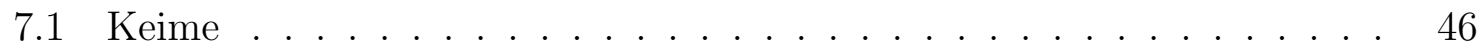

7.2 Kristallfragmente $\ldots \ldots \ldots \ldots \ldots \ldots$

7.3 Protokamin . . . . . . . . . . . . . . . . . . . 48

Z4 Dendritenwachstum . . . . . . . . . . . . . . . . . . . . . . . . 49

7.5 Markierung der Kamine $\ldots \ldots \ldots \ldots$

7.6 Kaminquerschnitt . . . . . . . . . . . . . . . . . . . . . 50

7.7 Bereich im Phasendiagramm, in dem Protokamine entstehen . . . . . . 51

7.8 Schichtwachstum bei unterschiedlichen Winkelgeschwindigkeiten . . . . 53

7.9 Einfluss der Unterkühlung aut die Wachtumsgeschwindigkeit . . . . . . 54

7.10 Wachtumskoeffizient in Abhängigkeit von der Winkelgeschwindigkeit . . 55

7.11 Dendrite . . . . . . . . . . . . . . . . . . . 56 
7.12 Konzentration . . . . . . . . . . . . . . . . . . . . . 58

7.13 Permeabilität aus Konzentrationsmessung _. . . . . . . . . . . . . 59

7.14 Kaminverteilung $\ldots \ldots \ldots \ldots \ldots$. . . . . . . . . . . . . 61

8.1 Fingerkonvektion . . . . . . . . . . . . . . . . . 63

8.2 Protoplumes . . . . . . . . . . . . . . . . . . . . . . . . . . . . . 63

$8.3 \quad$ Schattenbilder der Konvektionsentwicklung $\left(\Omega=0 \frac{\mathrm{rad}}{\mathrm{s}}\right) \ldots \ldots . . . \quad 65$

8.4 Plume-Größsen . . . . . . . . . . . . . . . . . . . . . . 66

8.5 Lokale Verdickungen im Plumestamm . . . . . . . . . . . . . . . 67

8.6 Konzentrations- und Geschwindigkeitsprofil eines Plumes . . . . . . . . 68

8.7 Höhe der Kristallschicht bei Einsatz der Plumeinstabilität . . . . . . . 69

8.8 Plumeanzah bei Konvektionseinsatz . . . . . . . . . . . . . . 71

8.9 Zeitliche Entwicklung der Plumekonvektion . . . . . . . . . . . . . 72

8.10 Schattenbilder der Konvektionsentwicklung $\left(\Omega=5,2 \frac{\mathrm{rad}}{\mathrm{s}}\right) \ldots \ldots . . .75$

8.11 Autbrechender Plume . . . . . . . . . . . . . . . . . . 76

8.12 Skizze der Tröpfcheninstabilität . . . . . . . . . . . . . . . 77

8.13 Tröpfcheninstabilität bei $\Omega=1,1 \frac{\mathrm{rad}}{\mathrm{s}} \ldots \ldots \ldots \ldots$. . . . . . 77

8.14 Diapin $\ldots \ldots \ldots \ldots \ldots$

8.15 Schattenbild eines gescherten Plumes . . . . . . . . . . . . . . 79

8.16 Höhe, in der die Tröpfcheninstabilität einsetzt . . . . . . . . . . . . 80

8.17 Schattenbilder von Plume- und Tröpfchenströmungsstrukturen . . . . . 81

9.1 Höhe, in der die Tröpfcheninstabilität einsetzt $\ldots \ldots$. . . . . . . 87 


\section{Tabellenverzeichnis}

3.1 Physikalische Parameter . . . . . . . . . . . . . . . . . . . . . . . . . 20

7.1 Dendritenabstand . . . . . . . . . . . . . . . . . . . . 55

7.2 Permeabilität und Porosität . . . . . . . . . . . . . . . . . 58

B.1 Experimente zum Einsatz der Plumekonvektion . . . . . . . . . . . . . 104

B.2 Experimente . . . . . . . . . . . . . . . . . . . . 105 


\section{Anhang A}

\section{Liste der verwendeten Symbole und Abkürzungen}

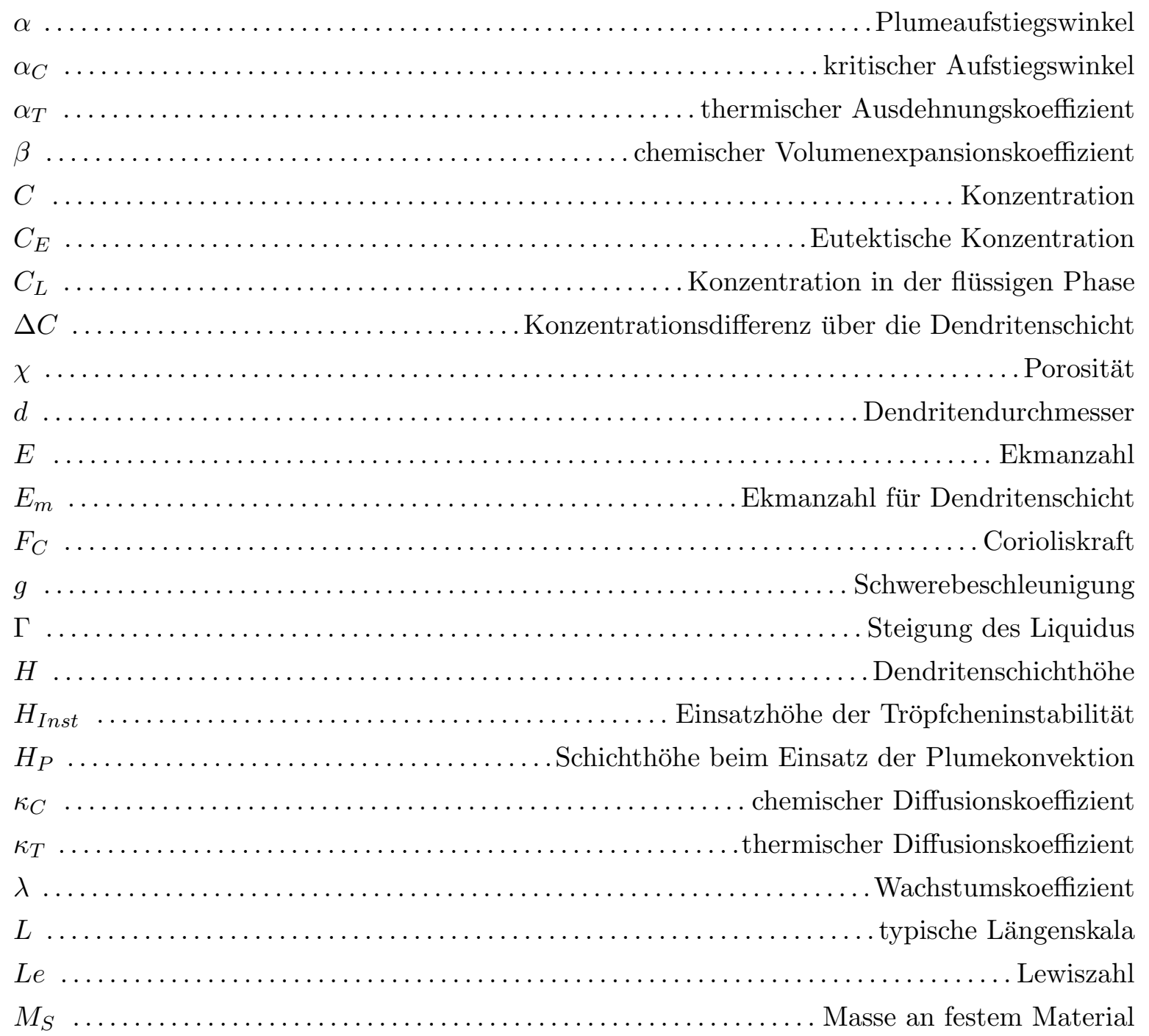




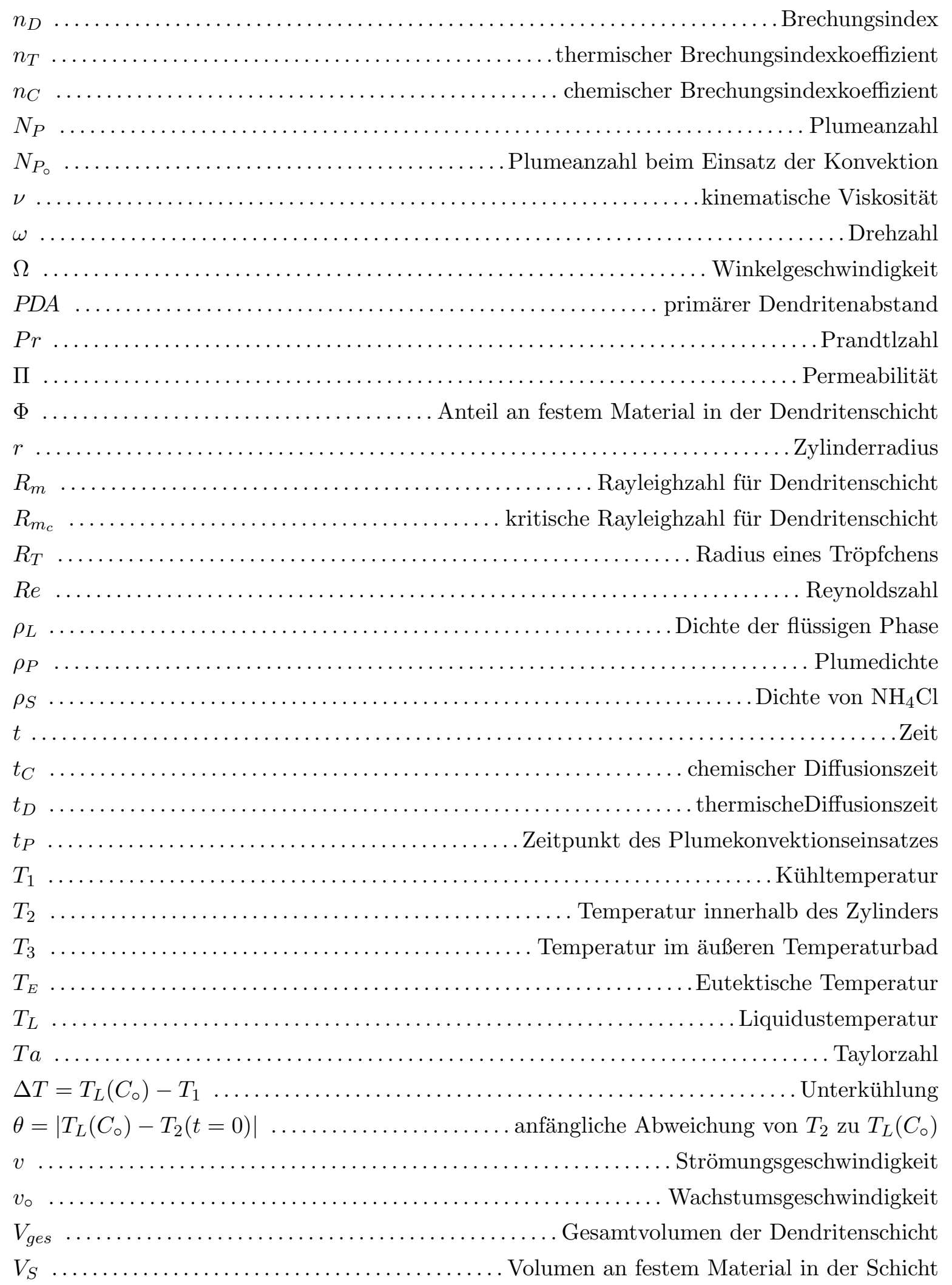




\section{Anhang B}

\section{Experimente}

\begin{tabular}{|l|c|c|c|c|c|c|c|c|}
\hline Exp. & $\mathrm{C}_{\circ}$ & $\Delta \mathrm{T}$ & $\theta$ & $\Omega$ & $\mathrm{H}_{P}$ & $\mathrm{~N}_{P_{\circ}}$ & $\mathrm{H}_{\text {Inst }}$ & $\mathrm{E}$ \\
\hline \hline C24R0DT12 & 24 & 12 & 12.7 & - & 10 & 17 & - & - \\
\hline C24R10DT12 & 24 & 12 & 11.3 & 1.1 & 10 & 10 & 26.5 & $7.5 \cdot 10^{-5}$ \\
\hline C24R60DT12 & 24 & 12 & 11.6 & 6.3 & 11 & 5 & 10 & $1.3 \cdot 10^{-5}$ \\
\hline C24R0DT15 & 24 & 15 & 10 & 0 & 11 & 15 & - & - \\
\hline C24R20DT15 & 24 & 15 & 3.9 & 2.1 & 10 & $\mathrm{ng}$ & 24 & $3.8 \cdot 10^{-5}$ \\
\hline C24R50DT15 & 24 & 15 & 9.8 & 5.3 & 9 & 11 & 1 & $1.5 \cdot 10^{-5}$ \\
\hline C24R0DT20 & 24 & 20 & 7.2 & 0 & 10.5 & $\mathrm{ng}$ & - & - \\
\hline C26R10DT5 & 26 & 4.5 & 5.6 & 1.1 & -1 & - & - & $7.5 \cdot 10^{-5}$ \\
\hline C26R10DT10 & 26 & 8.5 & 5.2 & 1.1 & 9 & 21 & $\mathrm{ng}$ & $7.5 \cdot 10^{-5}$ \\
\hline C26R30DT10 & 26 & 10.5 & 6 & 3.14 & 9 & 15 & 15 & $2.5 \cdot 10^{-5}$ \\
\hline C26R10DT25 & 26 & 24.5 & 15.6 & 1.1 & 10 & 35 & 25.5 & $7.5 \cdot 10^{-5}$ \\
\hline
\end{tabular}

Tabelle B.1: Experimente zum Einsatz der Plumekonvektion

Legende siehe Tabelle B.9

\footnotetext{
${ }^{1}$ Mit einer maximalen Schichthöhe von $H=7 \mathrm{~mm}$ konnte kein Einsatz der Plumekonvektion erreicht werden.
} 


\begin{tabular}{|l|c|c|c|c|c|c|c|c|}
\hline Exp. & $\mathrm{C}_{\circ}$ & $\Delta \mathrm{T}$ & $\theta$ & $\Omega$ & $\mathrm{H}_{P}$ & $\mathrm{~N}_{P_{\circ}}$ & $\mathrm{H}_{\text {Inst }}$ & $\mathrm{E}$ \\
\hline \hline C26R60DT10 & 26 & 9.5 & 1.2 & 6.3 & 11 & 2 & 8 & $1.3 \cdot 10^{-5}$ \\
\hline C26R0DT12 & 26 & 11.5 & 0.9 & 0 & $\mathrm{ng}$ & $\mathrm{ng}$ & - & - \\
\hline C26R40DT12 & 26 & 11.5 & 1.3 & 4.2 & $\mathrm{ng}$ & $\mathrm{ng}$ & $\mathrm{ng}$ & $1.9 \cdot 10^{-5}$ \\
\hline C26R90DT12 & 26 & 11.5 & 2.0 & 9.4 & $\mathrm{ng}$ & $\mathrm{ng}$ & $\mathrm{ng}$ & $8.5 \cdot 10^{-6}$ \\
\hline C26R0DT15a & 26 & 16,5 & 3.5 & 0 & $\mathrm{ng}$ & 4 & - & - \\
\hline C26R0DT15b & 26 & 15.5 & 0.9 & 0 & $\mathrm{ng}$ & 14 & - & - \\
\hline C26R0DT20a & 26 & 19.3 & 5.4 & 0 & $\mathrm{ng}$ & 10 & - & - \\
\hline C26R0DT20b & 26 & 19.8 & 5.3 & 0 & $\mathrm{ng}$ & 9 & - & - \\
\hline C26R0DT25a & 26 & 24.5 & 23.1 & 0 & $\mathrm{ng}$ & 45 & - & - \\
\hline C26R0DT25b & 26 & 24.5 & 1.5 & 0 & $\mathrm{ng}$ & $\mathrm{ng}$ & - & - \\
\hline C26R0DT25c & 26 & 24.5 & 1.4 & 0 & 10 & $\mathrm{ng}$ & - & - \\
\hline C26R20DT25a & 26 & 24.5 & 0.5 & 2.1 & 12 & 2 & 24 & $3.8 \cdot 10^{-5}$ \\
\hline C26R20DT25b & 26 & 24.5 & 0.2 & 2.1 & 9 & 4 & 25 & $3.8 \cdot 10^{-5}$ \\
\hline C26R50DT25a & 26 & 24.5 & 0.6 & 5.3 & 13 & $\mathrm{ng}$ & 14 & $1.5 \cdot 10^{-5}$ \\
\hline C26R50DT25b & 26 & 24.5 & 1.0 & 5.3 & $\mathrm{ng}$ & $\mathrm{ng}$ & $\mathrm{ng}$ & $1.5 \cdot 10^{-5}$ \\
\hline C26R60DT25 & 26 & 24.5 & 1.8 & 6.3 & 13 & 2 & 8.5 & $1.3 \cdot 10^{-5}$ \\
\hline C26R70DT25 & 26 & 24.5 & 1.1 & 7.3 & 10 & $\mathrm{ng}$ & 3.5 & $1.1 \cdot 10^{-5}$ \\
\hline C26R100DT25a & 26 & 0.2 & 24.5 & 10.5 & 12 & $\mathrm{ng}$ & 0.1 & $7.5 \cdot 10^{-6}$ \\
\hline C26R100DT25b & 26 & 0.8 & 24.5 & 10.5 & 10 & $\mathrm{ng}$ & 0.1 & $7.5 \cdot 10^{-6}$ \\
\hline C26R0DT35 & 26 & 36.5 & 5.3 & 0 & 11 & $\mathrm{ng}$ & - & - \\
\hline C28R0DT10 & 28 & 9 & 2.6 & 0 & 9.5 & $\mathrm{ng}$ & - & - \\
\hline C28R0DT15 & 28 & 15 & 1.1 & 0 & 10.5 & 24 & - & - \\
\hline C28R0DT20 & 28 & 19 & 2.1 & 0 & 8.5 & 23 & - & - \\
\hline C28R0DT25 & 28 & 24 & 1.1 & 0 & 9 & 20 & - & - \\
\hline C28R5DT25 & 28 & 24 & 0.5 & 0.5 & 10 & 12 & 47.8 & $1.5 \cdot 10^{-4}$ \\
\hline C28R20DT25 & 28 & 24 & 0.9 & 2.1 & 10 & 2 & 22 & $3.8 \cdot 10^{-5}$ \\
\hline C28R40DT25 & 28 & 24 & 1.9 & 4.2 & 8.5 & 2 & 14 & $1.9 \cdot 10^{-5}$ \\
\hline C28R80DT25 & 28 & 24 & 2.6 & 8.4 & 9 & $\mathrm{ng}$ & 0.3 & $9.5 \cdot 10^{-6}$ \\
\hline C28R90DT25 & 28 & 24 & 2.1 & 9.4 & 11 & $\mathrm{ng}$ & 0.1 & $8.5 \cdot 10^{-6}$ \\
\hline C35R60DT65 & 35 & 64.3 & 1.7 & 6.3 & 9 & $\mathrm{ng}$ & 9 & $1.3 \cdot 10^{-5}$ \\
\hline
\end{tabular}

Tabelle B.2: Resultate der Experimente

$\mathrm{C}_{\circ}\left[\right.$ [gew\% $\left.-\mathrm{NH}_{4} \mathrm{Cl}\right]:$ Anfangskonzentration; $\Delta T[\mathrm{~K}]$ : Unterkühlung; $\Omega[\mathrm{rad} / \mathrm{s}]:$ Winkelgeschwindigkeit; $\theta=\left|T_{L}\left(C_{\circ}\right)-T_{2}(t=0)\right|[K] ; H_{P}[m m]$ : Schichtdicke bei Einsatz der Plumekonvektion; $N_{P_{\circ}}$ : Plumeanzahl beim Einsatz der Konvektion; $H_{\text {Inst }}[\mathrm{mm}]$ : Höhe des Plumes bei Einsatz der Tröpfcheninstabilität; E: Ekmanzahl; ng: Wert wurde nicht ermittelt 


\section{Anhang C}

\section{Hardware}

\section{Umwälzthermostate}

- COLORA: K3 (Colora Messtechnik GmbH, Lorch)

Arbeitstemperatur: $+40 \ldots+100^{\circ} \mathrm{C}$ (ohne Kühlung)

$+20 \ldots+100^{\circ} \mathrm{C} \quad$ (mit Kühlung)

Betriebsbereich: $-10 \ldots+100^{\circ} \mathrm{C}$

Temperaturkonst.: $\pm 0,01 \mathrm{~K}$

Förderstrom: 18 l/min

Badvolumen: 3...3,5 l

- HAAKE: DC50-B3 (Gebr. Haake GmbH, Karlsruhe)

Arbeitstemperatur: $+35 \ldots+200^{\circ} \mathrm{C}$ (ohne Kühlung)

$-50 \ldots+200^{\circ} \mathrm{C} \quad$ (mit Kühlung)

Temperaturkonst.: $\pm 0,01 \mathrm{~K}$

Förderstrom: 12,5 l/min

Badvolumen: 31

Schnittstelle: RS 232 C

Anschluß für ext. Temperaturfühler: HAAKE DTT (PT100)

\section{Umwälzkryostat}

COLORA: KT140K (Colora Messtechnik GmbH, Lorch)

Arbeitstemperatur: $-40 \ldots+20^{\circ} \mathrm{C}$

Temperaturauflösung: $0,1 \mathrm{~K}$ (Anzeige)

Temperaturkonst.: $\pm 0,02 \mathrm{~K}$

Förderstrom: $20 \mathrm{l} / \mathrm{min}$

Badvolumen: 11...12 l

\section{Digitalthermometer}

FLUKE: 2161 A (Fluke GmbH, Düsseldorf )

Thermoelement Typ K (NiCr/NiAl)

Temperaturbereich: $-99,8 \ldots+999,8^{\circ} \mathrm{C}$

Auflösung (Genauigkeit): $\pm 0,2 \mathrm{~K}$

Eingangskanäle: 10, erweiterbar auf 20 


\section{Waage}

SARTORIUS: LC4201S (Sartorius AG, Göttingen)

Maximalgewicht: $4200 \mathrm{~g}$

Auflösung (Genauigkeit): $\pm 0,01 \mathrm{~g}$

\section{Refraktometer}

KRÜSS: AR3 (Krüss Optronics GmbH, Hamburg)

Typ: Abbe

Messbereich: Brechungsindex $1,3000 \ldots 1,7200$

Brix $\quad 0,0 \ldots 95,0 \%$

Auflösung (Genauigkeit): $0,0001 \mathrm{n}_{D}$

$$
0,1 \%
$$

Messprinzip: Beobachtung des Grenzwinkels in durchfallendem Licht (durchsichtige Flü ssigkeiten) bei streifendem Eintritt

\section{Schrittmotorsteuerung}

ISEL: CNC-Contoller C116 (ISEL-Automation, Eiterfeld)

- Controller zur Ansteuerung von bis zu Drei Schrittmotoren

- 3-dim. CNC-Betriebssystem

- max. Antriebsleistung: $110 \mathrm{Ncm}$

\section{Schrittmotor}

ISEL: MS-160 (ISEL-Automation, Eiterfeld)

Haltemoment: bipolar $160 \mathrm{Ncm}$ unipolar $130 \mathrm{Ncm}$

Schrittwinkel: Halbschritt $0,9^{\circ}$

Radiallast: $33 \mathrm{~N}$

\section{Lack}

Plastik-Spray 70 (Kontakt-Chemie GmbH, Rastatt)

Hochisolierender, glasklarer Acyl-Harzlack

Beständig gegen Säuren und Laugen

\section{CCD-Kamera}

JAI: CV-M10BXC (DBS GmbH, Bremen)

Bildsensor: 1/2" Progr. Interline, monochrom

Bildelemente: $782(\mathrm{H}) \times 582(\mathrm{~V})$

Pixelgröße: quadr. $8,3 \mu \mathrm{m} \times 8,3 \mu \mathrm{m}$

Auflösung: 600 Linien

Abtastsystem: Non-Interlace/ Interlace

Ext. Synch. Eingang (ext. Trigger) 
Videoausgang: CCIR, non-standard

Objektiv-Anschluß: C-Mount

\section{Objektiv}

COMPUTAR: H670812 (DBS GmbH, Bremen)

manuelles Zoomobjektiv

Brennweite: 8-48 mm

Anschluß: C-Mount

\section{Zwischenringsatz}

VM100 (DBS GmbH, Bremen)

Verkürzungung des minimalen Objektabstandes

Dicke: 0,5 mm, $1 \mathrm{~mm}, 10 \mathrm{~mm}, 20 \mathrm{~mm}$ und $40 \mathrm{~mm}$

\section{Frame-Grabber}

ELTEC: PC_EYE1 (DBS GmbH, Bremen)

Bus: PCI 32 Bit

Analoge Eingänge: 4 für Monochrom (8 Bit)

Digitale Eingänge: Pixel Clock (TTL), Trigger zum asynchr. Reset

A/D-Wandlung (Abtastrate): CCIR 14,3 MHz

Rauschen: 1 LSB RMS

Treiber-Software: Win NT

Empfohlene Compiler: C-Compiler (Borland, Microsoft, Watcom)

Mitgelieferte Applikations-Software: Grab\&View Light 


\section{Danksagung}

Herrn Prof. Dr. Ulrich Christensen danke ich für die Ermöglichung dieser Arbeit am Institut für Geophysik in Göttingen und seine freundliche Unterstützung während der Entstehung.

Herrn Prof. Dr. Helmut Eckelmann möchte ich für die Übernahme des Korreferats danken.

Dr. Moritz Heimpel verdanke ich die Grundidee zu dieser Arbeit und viele weiterführende Anregungen.

Den Mitarbeitern der feinmechanischen Werkstatt des Instituts, Wilfried Steinhoff, Rudolf Deppe und Jürgen Cruse gilt mein besonderer Dank für den Bau der Rotationsapparatur und den Einblick in ihre Arbeitstechniken.

Johannes Wicht, Sebastian Fähler, Dirk Neumeister und Sigrid Claßen bin ich sehr dankbar für die sorgfältige und prompte Durchsicht meiner Arbeit. Johannes und Sebastian verdanke ich zudem lange und fruchtbare Diskussionen. Sigrid hat nicht davor zurück geschreckt auch noch die letzten Seiten zu korrigieren; nochmals vielen Dank an alle.

Sebastian Fähler verdankt mir den arbeitsreichsten Urlaub seines Lebens und dafür werd ich ihm wohl nie genug danken können.

Dem Graduiertenkolleg „Strömungsinstabilitäten und Turbulenz" der Universität Göttingen verdanke ich nicht nur die finanzielle Unterstützung in den ersten Jahren meiner Promotion, sondern auch die Gelegenheit in andere strömungsphysikalische Gebiete hinein zuriechen.

Meiner Arbeitsgruppe und allen anderen Mitarbeitern des Instituts gilt ein herzliches Dankeschön für das gute Arbeitsklima. Zudem besitzt das Institut noch eine hervorragende Sekretärin, die nicht nur bei allen bürokratischen Dingen des Lebens weiterhilft sondern auch ein offenes Ohr für alles andere hat - Danke Erika! An dieser Stelle soll auch einmal dem besten Hausmeister der Welt, Manfred Herden, für seinen gut gefüllten Keller gedankt werden.

Bei meiner Mutter möchte ich mich ganz herzlich für ihre langjährige Unterstützung und viele, viele Care-Pakete bedanken. 


\title{
Lebenslauf
}

\author{
Geburtstag 9. Januar 1967 \\ Geburtsort Rheydt, jetzt Mönchengladbach \\ Familienstand ledig \\ Nationalität deutsch \\ 1973 - $1977 \quad$ Katholische Grundschule Güdderath \\ 1977 - $1986 \quad$ Hugo-Junkers Gymnasium in Rheydt \\ 2. Juni $1986 \quad$ Abitur \\ 1986 - $1988 \quad$ Studium der Elektrotechnik in Aachen \\ 1988 - 1995 Studium der Physik in Göttingen \\ 1992 - 1995 Diplomarbeit am Institut für Geophysik in Göttingen \\ bei Prof. Dr. U. Christensen zum Thema: \\ „Laborexperimente zur Untersuchung \\ der Nusselt- und Rayleighzahl-Beziehung \\ für transiente thermische Konvektion“ \\ 27. April 1995 Diplom \\ 1995 - $1997 \quad$ Stipendiatin des Graduiertenkollegs \\ „Strömungsinstabilitäten und Turbulenz“ \\ an der Georg-August-Universität zu Göttingen \\ ab 1997 assoziiertes Mitglied des Graduiertenkollegs \\ 1995 Beginn der Dissertation am Institut für Geophysik in Göttingen \\ bei Prof. Dr. U. Christensen
}

